Florida International University FIU Digital Commons

FIU Electronic Theses and Dissertations

University Graduate School

6-29-2017

\title{
Mobility Analysis and Management for Heterogeneous Networks
}

Karthik Vasudeva

Florida International University, kvasu001@fiu.edu

DOI: $10.25148 /$ etd.FIDC001983

Follow this and additional works at: https://digitalcommons.fiu.edu/etd

Part of the Systems and Communications Commons

\section{Recommended Citation}

Vasudeva, Karthik, "Mobility Analysis and Management for Heterogeneous Networks" (2017). FIU Electronic Theses and Dissertations. 3348.

https://digitalcommons.fiu.edu/etd/3348

This work is brought to you for free and open access by the University Graduate School at FIU Digital Commons. It has been accepted for inclusion in FIU Electronic Theses and Dissertations by an authorized administrator of FIU Digital Commons. For more information, please contact dcc@fiu.edu. 


\section{FLORIDA INTERNATIONAL UNIVERSITY \\ Miami, Florida}

\section{MOBILITY ANALYSIS AND MANAGEMENT FOR HETEROGENEOUS NETWORKS}

A dissertation submitted in partial fulfillment of the requirements for the degree of DOCTOR OF PHILOSOPHY

in

ELECTRICAL ENGINEERING

by

Karthik Vasudeva 


\section{To: Interim Dean Ranu Jung}

College of Engineering and Computing

This dissertation, written by Karthik Vasudeva, and entitled Mobility Analysis and Management for Heterogeneous Networks, having been approved in respect to style and intellectual content, is referred to you for judgment.

We have read this dissertation and recommend that it be approved.

$\begin{array}{r}\hline \text { Jean H. Andrian. } \\ \hline \text { Kang K. Yen. } \\ \hline \text { Deng Pan. } \\ \hline \text { David López-Pérez. } \\ \hline \text { İsmail Güvenç, Major Professor. }\end{array}$

Date of Defense: June 11, 2017

The dissertation of Karthik Vasudeva is approved.

Interim Dean Ranu Jung College of Engineering and Computing

Andrés G. Gil

Vice President for Research and Economic Development and Dean of the University Graduate School

Florida International University, 2017 


\section{ACKNOWLEDGMENTS}

First and foremost, I would like to express my sincere gratitude to my advisor Dr. Ismail Guvenc for his endless support during my Ph.D. program. His motivation, patience and concern has pushed me to significantly improve critical thinking ability and instilled confidence to face challenges during my research. He always guided me through obstacles that came along during my Ph.D. study. Further, i would like to express my sincere thanks to all postdoctoral scholars with whom i have worked and their constant encouragement and guidance has helped me to tackle several critical problems during my Ph.D program and also thank my dissertation committee members for the feedback. Next my heartfelt thanks goes to all my fellow labmates and friends for their support and encouragement. Last but not the least, special thanks goes to my family who patiently believed in me and their endless prayers helped me during the difficult times of my Ph.D. study.

This research work was supported in part by the U.S. National Science Foundation under the Grant CNS-1406968: NeTS: JUNO: "Energy-Efficient Hyper-Dense Wireless Networks with Trillions of Devices". 


\section{ABSTRACT OF THE DISSERTATION \\ MOBILITY ANALYSIS AND MANAGEMENT FOR HETEROGENEOUS NETWORKS \\ by \\ Karthik Vasudeva \\ Florida International University, 2017 \\ Miami, Florida \\ Professor İsmail Güvenç, Major Professor}

The global mobile data traffic has increased tremendously in the last decade due to the technological advancement in smartphones. Their endless usage and bandwidthintensive applications will saturate current $4 \mathrm{G}$ technologies and has motivated the need for concrete research in order to sustain the mounting data traffic demand. In this regard, the network densification has shown to be a promising direction to cope with the capacity demands in future $5 \mathrm{G}$ wireless networks. The basic idea is to deploy several low power radio access nodes called small cells closer to the users on the existing large radio foot print of macrocells, and this constitutes a heterogeneous network (HetNet).

However, there are many challenges that operators face with the dense HetNet deployment. The mobility management becomes a challenging task due to triggering of frequent handovers when a user moves across the network coverage areas. When there are fewer users associated in certain small cells, this can lead to significant increase in the energy consumption. Intelligently switching them to low energy consumption modes or turning them off without seriously degrading user performance is desirable in order to improve the energy savings in HetNets. This dynamic power level switching in the small cells, however, may cause unnecessary handovers, and it becomes important to ensure energy savings without compromising handover per- 
formance. Finally, it is important to evaluate mobility management schemes in real network deployments, in order to find any problems affecting the quality of service (QoS) of the users. The research presented in this dissertation aims to address these challenges.

First, to tackle the mobility management issue, we develop a closed form, analytical model to study the handover and ping-pong performance as a function of network parameters in the small cells, and verify its performance using simulations. Secondly, we incorporate fuzzy logic based game-theoretic framework to address and examine the energy efficiency improvements in HetNets. In addition, we design fuzzy inference rules for handover decisions and target base station selection is performed through a fuzzy ranking technique in order to enhance the mobility robustness, while also considering energy/spectral efficiency. Finally, we evaluate the mobility performance by carrying out drive test in an existing $4 \mathrm{G}$ long term evolution (LTE) network deployment using software defined radios (SDR). This helps to obtain network quality information in order to find any problems affecting the QoS of the users. 


\section{TABLE OF CONTENTS}

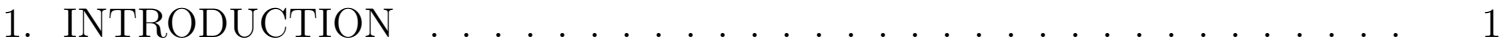

1.1 Contribution and Organization $\ldots \ldots \ldots \ldots . \ldots \ldots$

2. Theoretical background and Related work _... . . . . . . . 5

2.1 Mobility Management in Cellular Network . . . . . . . . . . . . . 5

2.1.1 Related Works on Handover Performance Analysis . . . . . . . . . . 6

2.2 Mobility Management for Energy Efficient Operation in HetNets . . . . . 7

2.3 Experimental Study on Mobility Performance in Real LTE scenarios using

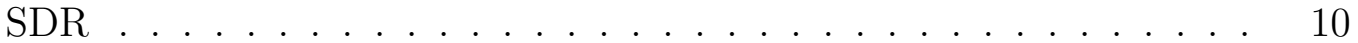

3. Handover failure analysis in Heterogeneous Networks . . . . . . . . . . . . 12

3.1 Review of the Handover Process in LTE . . . . . . . . . . . . . . . . 13

3.1 .1 Handover Failures and Ping-Pongs . . . . . . . . . . . . . . . 14

3.1.2 Handover Measurements Procedure in LTE. . . . . . . . . . . . . . . 15

3.2 Geometric Model for Handover Performance Analysis . . . . . . . . . . . 18

3.2.1 Modeling the Handover Trigger Locations . . . . . . . . . . . . . . . . 21

3.2 .2 Modeling the UEs' Sojourn Times . . . . . . . . . . . . . . . . 23

3.3 Handover Failure Analysis without Fading . . . . . . . . . . . . . 25

3.3 .1 No Handover Probability for MUEs . . . . . . . . . . . . . . . . . 26

3.3 .2 HF Probability for MUEs . . . . . . . . . . . . . . . . . . . . . . . . . 29

3.3 .3 HF Probability for PUEs . . . . . . . . . . . . . . . . . . 31

3.4 Handover Failure Analysis with Fading . . . . . . . . . . . . . . . 34

3.4.1 Modeling the Handover Trigger Locations in the Fading Scenario . . . 34

3.4 .2 HF Probability Analysis for MUEs . . . . . . . . . . . . . . . 36

3.4 .3 HF Probability Analysis for PUEs . . . . . . . . . . . . . . . 37

3.5 Numerical Results . . . . . . . . . . . . . . . . . . . . . . . . . . . 38

3.5.1 Results with No Fading . . . . . . . . . . . . . . . . . . . . . . . . . 39

3.5 .2 Results with Fading . . . . . . . . . . . . . . . . . . 42

4. Fuzzy Based Game Theoretic Mobility Management for Energy Efficient Op-

eration in HetNets . . . . . . . . . . . . . . . . . . . 45

4.1 Proposed Game Theoretic Approach . . . . . . . . . . . . . . . . . . 47

4.1.1 Regret Based Game Theoretic Learning Scheme . . . . . . . . . . . . 49

4.2 Context-Aware Fuzzy Handover Scheme . . . . . . . . . . . . . . . . . 51

4.2 .1 Handover Necessity Decision . . . . . . . . . . . . . . . . . . . . 51

4.2 .2 Target BS Selection . . . . . . . . . . . . . . . . . 55

4.3 Simulation Results . . . . . . . . . . . . . . . . . . . 55

4.3 .1 Impact on BS Energy Consumption . . . . . . . . . . . . . . . 57

4.3.2 Impact on Ping Pong Performance . . . . . . . . . . . . . . . . . . . 59

4.3.3 Impact on BS Throughput Performance . . . . . . . . . . . . . . . 61 
5. An Experimental Study on Mobility Performance in Real LTE scenarios using Software Defined Radio . . . . . . . . . . . . . . . . . . . . . 62

5.1 Introduction . . . . . . . . . . . . . . . . . . . 62

5.2 Measurement Setup for signal recording in the drive test . . . . . . . 63

5.3 Post Processing of Drive Test Measurements in Matlab . . . . . . . . . . 65

5.3 .1 Resampling . . . . . . . . . . . . . . . . . 66

5.3.2 Frequency Offset Estimation and Correction . . . . . . . . . . . 66

5.3.3 LTE Cell Search and Timing Synchronization . . . . . . . . . . . . 69

5.3 .4 OFDM Demodulation . . . . . . . . . . . . . . . . 70

5.3 .5 Channel Estimation . . . . . . . . . . . . . . . . . . 72

5.3 .6 Extract PBCH Resources . . . . . . . . . . . . . . . . . . 73

5.3 .7 PBCH Decoding . . . . . . . . . . . . . . . . . . . 73

5.3.8 Decoding SIB1 to uniquely identify the base station . . . . . . . . 76

5.3.9 Decoding the SIB1 information using ASN.1 compiler . . . . . . . . . . 80

5.4 Experimental Results . . . . . . . . . . . . . . . . . . . 82

6. Concluding Remarks . . . . . . . . . . . . . . . . 85

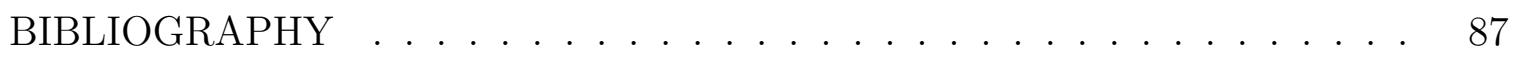

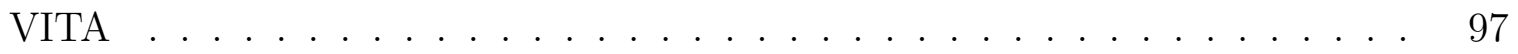




\section{LIST OF FIGURES}

FIGURE

PAGE

1.1 Heterogeneous network scenario. . . . . . . . . . . . . . . . 1

$2.1 \mathrm{ON} / \mathrm{OFF}$ energy saving technique. . . . . . . . . . . . . 8

3.1 Signalling overhead problem in the dense HetNet scenario. . . . . . . . . 12

3.2 Handover failure problem in HetNets due to small cell size. . . . . . . . 13

3.3 Modeling of the HF based on 3GPP mobility management studies for HetNets [83]. . . . . . . . . . . . . . . . . . . . . 14

3.4 Handover measurement performed by the UE through two different L1 filtering approaches. . . . . . . . . . . . . . . 16

3.5 The bouncing ring UE mobility model from [83]. . . . . . . . . . . . . . 19

3.6 Handover trigger and HF locations in an example picocell using a 3GPPcompliant simulator. The MBS is located at $(1500,1500) \mathrm{m}$ and has three sectors $\left(T_{\mathrm{d}}=200 \mathrm{~ms}\right)[32] . \ldots \ldots \ldots$

3.7 Geometric handover model to analyze HFs for MUEs and PUEs, and considers the effects of L3 filtering. The parameter $l(\theta)$ refers to the length of the segment for the UE trajectory, for which the segment becomes a chord of the picocell coverage circle. . . . . . . . . . .

3.8 Handover offset distance histograms for $v=60 \mathrm{~km} / \mathrm{hr}$ in Case- 1 . For the scenario of $T_{\mathrm{d}}=200 \mathrm{~ms}$ adopted in 3GPP LTE, the handover offset distance histogram can be reasonably modeled using a uniform distribution. . . . . . . . . . . . . . . . .

3.9 Different interpretations of randomness of a chord in a circle . . . . . .

3.10 PDFs of the chord length for the three different approaches in Bertrand's Paradox, and the histogram of the chord length for the bouncing-ring simulations in Fig. 3.5. . . . . . . . . . . . . .

3.11 Handover locations in the fast-fading and shadowing scenario (Case 3) for UE velocity $v=60 \mathrm{~km} / \mathrm{h}$ and $T_{\mathrm{d}}=40 \mathrm{~ms}$. . . . . . . . . . . 35

3.12 Handover offset distance histograms for $T_{\mathrm{d}}=40 \mathrm{~ms}$ in fading scenario. .

3.13 Theoretical (lines) and simulated (markers) no fading results as a function of UE velocity for $R=64 \mathrm{~m}, r_{\mathrm{m}}=50 \mathrm{~m}$ and $r_{\mathrm{p}}=78 \mathrm{~m}$ in the case of non-overlapping handover filtering process $[7] \ldots \ldots . . . .$.

3.14 Theoretical (lines) and simulated (markers) no fading results as a function of UE velocity for $R=64 \mathrm{~m}, r_{\mathrm{m}}=50 \mathrm{~m}$ and $r_{\mathrm{p}}=78 \mathrm{~m}$. . . . . . 
3.15 Ping-pong results as a function of UE velocity for $R=64 \mathrm{~m}, r_{\mathrm{m}}=50 \mathrm{~m}$ and $r_{\mathrm{p}}=78 \mathrm{~m} \ldots \ldots \ldots \ldots \ldots$

3.16 Theoretical (lines) and simulated (markers) no fading results as a function of UE velocity for $R=64 \mathrm{~m}, r_{\mathrm{m}}=50 \mathrm{~m}$ and $r_{\mathrm{p}}=78 \mathrm{~m} . \ldots$.

3.17 Simulation plots for fading ping-pong probabilities as a function of UE velocity for $R=64 \mathrm{~m}, r_{\mathrm{m}}=50 \mathrm{~m}$ and $r_{\mathrm{p}}=78 \mathrm{~m}$. . . . . . . .

4.1 Two-tier HetNet with small cells and mobile users. . . . . . . . . . . . . 45

4.2 The proposed fuzzy logic controller for the handover decisions, composed of three fuzzy inference systems (FIS) . . . . . . . . . . . . . . . 51

4.3 Membership functions for different user velocities. . . . . . . . . . . . 53

4.4 Design of the handover FLC for the MBS. . . . . . . . . . . . . . . 54

4.5 Design of the handover FLC for the SBS. . . . . . . . . . . . . 55

4.6 Proposed fuzzy logic handover scheme: handover necessity decision (left), and target BS selection (right). . . . . . . . . 56

4.7 Two tier HetNet where MBS is located at the origin and circles represent the coverage of the SBSs. . . . . . . . . . . . . . . 56

4.8 Energy consumption versus time $(15$ UEs). . . . . . . . . . . . . . . . 58

4.9 Average SBS energy consumption versus number of users $\left(\mathrm{N}_{\mathrm{SBS}}=7\right)$. . 58

4.10 Average SBS load as a function of the number of users $\left(\mathrm{N}_{\mathrm{SBS}}=7\right)$. . . $\quad 59$

4.11 Average ping-pong handover rate as a function of the number of users

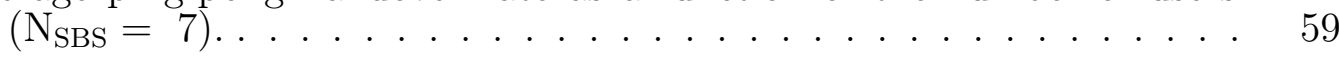

4.12 Average ping-pong handover rate as a function of user velocity (15 UEs). 60

4.13 SBS throughput results. . . . . . . . . . . . . . . . . 61

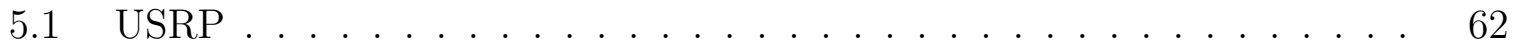

5.2 Signal recording block diagram in LabVIEW. . . . . . . . . . . . . . . 64

5.3 RMC data format . . . . . . . . . . . . . . . . . . 64

5.4 The recorded signal using Labview are processed in Matlab to obtain MIB bits. After resampling to $1.92 \mathrm{MHz}$ frequency and timing synchronization is performed, the LTE grid is constructed and PBCH symbols are decoded to obtain MIB bits. . . . . . . . . . . . . . . .

5.5 Scheduling of MIB information in time domain. . . . . . . . . . . . 67 
5.6 The spectrum of the LTE signal with $739 \mathrm{MHz}$ carrier frequency sampled at $16.67 \mathrm{MHz}$ using USRP N210 in Labview. . . . . . . . . . . . . .

5.7 The spectrum of the resampled LTE signal with $1.92 \mathrm{MHz}$ sampling rate for MIB decoding. Decoding the central 6 resource blocks is possib;e with this sampling rate. . . . . . . . . . . . . . .

5.8 The product of the resampled signal and its 128 sample shifted version averaged over all available slots in the received signal. The peaks occur at locations locations of $\mathrm{CP}$ and these points are used for frequency offset estimation. . . . . . . . . . . . . . . . .

5.9 The magnitude of PSS and SSS signal for PCI=0. In order to find the PCI of the received signal SSS and PSS for all PCIs can be generated and correlated with the received signal. The PSS, SSS, and delay combination that gives maximum correlation is chosen for possible PCI candidate. . . . . . . . . . . . . . . . . .

5.10 The PSS and SSS signal when PCI=134 gives maximum correlation of 0.38 at delay 10306 . For the received sinal this is the maximum correlation value among all PSS, SSS, and delay combinations. . . . . .

5.11 Received grid after OFDM demodulation. For the central 6 resource block, the grid contains 72 frequency bins. Here the grid is shown for the first subframe containing 14 OFDM symbols. The locations of PSS, SSS, and PBCH are also shown. . . . . . . . . . . . .

5.12 CRS symbols from transmit antenna . . . . . . . . . . . . . 72

5.13 The magnitude of the estimate of the channel from transmitter antenna 1. The CRS symbols are used and interpolated to get this estimate.

5.14 The magnitude of the estimate of the channel from transmitter antenna 2. 74

5.15 The locations and magnitudes of the PBCH symbols in subframe 0. . . 74

5.16 The magnitude of channel estimate from transmitter antenna 1 at $\mathrm{PBCH}$ symbols locations. . . . . . . . . . . . . . .

5.17 The magnitude of channel estimate from transmitter antenna 2 at $\mathrm{PBCH}$ symbols locations. . . . . . . . . . . . . . 76

5.18 Scheduling of SIB1 information in time domain. . . . . . . . . . . . . . 77

5.19 Decoding of SIB1 information. . . . . . . . . . . . . . . . 77

5.20 Decoded BCCH-DL-SCH message from SIB1 bits . . . . . . . . . . . 81

5.21 Drive test results around NCSU campus. . . . . . . . . . . . . . 82

5.22 RSRP plots for the strongest base station. . . . . . . . . . . . 83 
5.23 Drive test results. . . . . . . . . . . . . . . . . . . . 83

5.24 Distribution of base stations in the downtown and country side drive test scenarios. . . . . . . . . . . . . . . . . 


\section{CHAPTER 1 \\ INTRODUCTION}

The new generation of wireless user equipments (UEs) and bandwidth-intensive applications have made data traffic to increase in an exponential manner, straining current cellular networks to a breaking point. According to Cisco's Global Mobile Data Traffic Forecast Update [1], the mobile data traffic has grown over 18-fold between 2011 and 2016, and 60 percent was accounted from mobile video. It is expected to continue to rise and traffic generated by smartphone to increase by 10 times by 2022 [2].

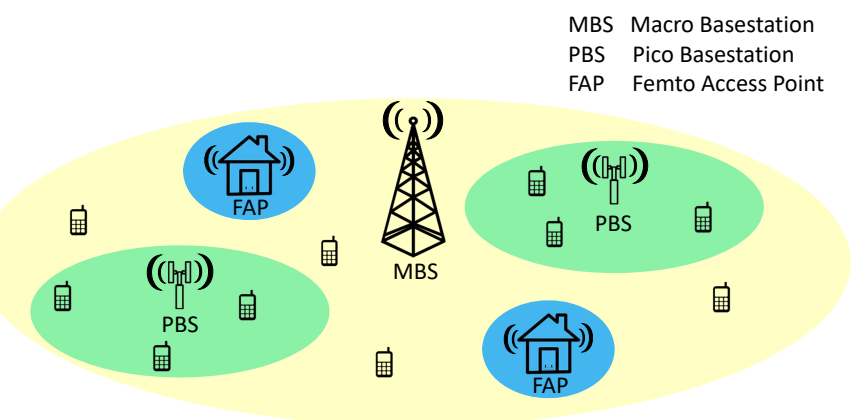

Figure 1.1: Heterogeneous network scenario.

Heterogeneous networks (HetNets) shown in Fig. 1.1, which consist of traditional macrocells overlaid with small cells (e.g. picocells, femtocells etc.) have shown to be a promising solution to cope with this wireless capacity crunch problem [3]. Due to their promising characteristics, HetNets have gained much momentum in the wireless industry and research community during the past several years. For instance, there have been dedicated study and work items in the 3rd generation partnership project (3GPP) related to HetNet deployments [4]. Their evolutions are also one of the major technology components that are being considered for 5G wireless systems [5].

Despite their promising features, HetNets have introduced new challenges. The dissertation mainly focus on the following challenges. Firstly the mobility management becomes a challenging task. Handover is the main process that supports seam- 
less connectivity of UEs among base stations (BSs). Due to increased number of BSs in a HetNet, it is more challenging to support seamless mobility of UEs in this type of scenario where handovers may fail more frequently [6]. In particular, using the same set of handover parameters of a traditional macrocellular network for a HetNet will degrade the mobility performance of UEs [7].

Secondly, the uncoordinated and massive deployment of small cells can lead to significant increase in energy consumption due to the energy costs of cells even when they have no associated users. It is expected that the carbon foot print of the mobile communication sector will increase up to twofold by 2020 from 2013, which is 201 Mega-tons of $\mathrm{CO}_{2}$ emissions. Therefore, reducing the energy consumption has become a major priority in the recent years.

Lastly, the present LTE systems supports only hard handover which means ongoing connection of users from source cell is broken before making a handover to the target cell. In the dense network and high speed user scenarios, this interruption can severely degrade the user quality of service (QoS).

\subsection{Contribution and Organization}

This research work aim to address the issues presented in the previous section and the main contribution of the dissertation can be explained as follows.

1. Handover Failure Analysis in LTE HetNets: In order to study the mobility management problem, a geometric handover model is introduced for analyzing handover failures in small cell deployments. Handover trigger locations at a picocell, and radio link failure locations at a macrocell and a picocell are modeled using co-centric circles. Considering a linear mobility model for UEs, closed form expressions for handover failure probabilities in the macrocell and

a picocell as function of important network parameters were obtained. The 
analysis is then extended to fast-fading and shadowing scenarios, where the statistics related to channel fading are extracted from a $3 \mathrm{GPP}$ compliant system level simulator in order to obtain semi-analytic expressions for handover failure probabilities. All theoretical results are validated through simulations, where impact of different parameters on $\mathrm{HF}$ and ping-pong probabilities are investigated.

\section{Fuzzy-Based Game Theoretic Mobility Management for Energy Ef-} ficient Operation in HetNets: We introduce a fuzzy logic based game theoretic approach for dynamically placing cells into sleep mode while also considering throughput and handover performance. We aim to optimize the fuzzy rules to obtain ideal transmission BS power levels for serving the UEs. Fur-

thermore, a context-aware fuzzy handover scheme is proposed consisting of two modules: 1) handover decision and 2) target BS selection in order to minimize unnecessary frequent handovers caused due to the dynamic power level switching of the BS. We develop fuzzy decision rules for handovers and target base station selection while simultaneously considering the energy/spectral efficiency and handover performance. The base station selection is performed using the fuzzy technique for order of preference by similarity to ideal solution (FTOPSIS) ranking method during the target BS selection stage of the handover process.

3. Mobility Performance Investigation in Real LTE scenarios using Software Defined Radio: The mobility performance of the real LTE networks is studied by performing extensive drive test and record measurements in LTE band using and software defined radio called universal software radio peripheral (USRP). The recorded measurements are post processed to determine the the coverage of the BS and study the effect of user velocity on the handover performance of the network. 
The rest of the dissertation is organized as follows. The Chapter 2 explains the theoretical background and related work, in Chapter 3 we provide the model to analyze handover failure in small cells, the Chapter 4 presents the fuzzy based game theoretic framework and context aware handover scheme for energy efficient operation and mobility robustness optimization respectively. In Chapter 5, the mobility performance in real LTE scenarios is studied through post processing of drive test measurements performed using software defined radio and the last section provides the concluding remarks. 


\section{CHAPTER 2}

\section{THEORETICAL BACKGROUND AND RELATED WORK}

\subsection{Mobility Management in Cellular Network}

Mobility management is a critical function of an cellular network, which enable the users to be seamlessly connected across the network through handover process. Handover performance has been studied for homogeneous [8-14] and heterogeneous [15-25] network deployments in the literature. In homogeneous networks, the authors in [8] use computer simulations to investigate the handover performance of LTE networks, considering different measurement filtering parameters at the UE. Novel self-organizing handover management techniques are proposed in [9-12], where the network autonomously configures the mobility management parameters for different scenarios, thereby improving the handover performance of the homogeneous cellular network. Handover parameters (e.g. time-to-trigger (TTT), hysteresis threshold, etc.) are optimized in [15] to achieve robust and seamless mobility of UEs in a HetNet scenario.

In [16], mobility performance of UEs is evaluated in a co-channel small cell networks scenario. When the density of the small cell increases, switching off the macro cell is shown to provide seamless mobility for the low speed UEs, while it degrades the handover performance for the high speed UEs [17]. Furthermore, in [18] authors show that using intercell interference coordination (ICIC) techniques can enhance the handover performance for both low and high speed UEs. Mobility state estimation is performed in $[21,26,27]$ to estimate the velocity of the UEs and keep the high speed UEs connected to the macrocells and the low speed UEs connected to the pic-

ocells, thereby enhancing the handover performance of the UEs. In [22], mobility performance is analyzed with and without inter-site carrier aggregation for macro 
and pico cells deployed on different carrier frequencies. The authors in $[23,24]$ aim to improve the mobility performance of UEs across different network types such as WiFi, WiMAX, LTE, and Bluetooth, by performing a vertical handoff (VHO).

\subsubsection{Related Works on Handover Performance Analysis}

Despite all these related work on mobility management in HetNets, there are only limited theoretical studies that analyze the handover performance in HetNet scenarios. In [28], the authors derive the handover rate and sojourn time of a UE for the Poisson-voronoi and hexagon cellular topologies. Expressions for call block and drop probabilities in a small cell scenario are derived in [29]. Theoretical analysis for handover performance optimization is done in [30] to quantify the user performance as a function of user mobility parameters. In [31], a mathematical framework was proposed to model the handover measurement function, and expressions were derived for measurement failure and best target cell. In [32], handover performance analysis was performed as a function of handover parameters, e.g. TTT and UE velocity, assuming the UE performs measurements continuously with no delay.

The main goal of this research work [33-35] is to introduce a simple yet effective model for analyzing handover failures in small cell deployments, considering all important mobility management parameters of interest. Handover trigger locations at a picocell, and radio link failure locations at a macrocell and a picocell are modeled using co-centric circles. Considering a linear mobility model for UEs, HF probabilities for macrocell and picocell UEs are derived in closed form for various scenarios. The analysis is then extended to fast-fading and shadowing scenarios: relevant statistics in a fading scenario are extracted from a 3GPP compliant system level simulator to facilitate semi-analytic expressions for HF probabilities. All theoretical results are validated through simulations, where impact of different parameters on $\mathrm{HF}$ and ping-pong probabilities are investigated. 


\subsection{Mobility Management for Energy Efficient Operation in}

\section{HetNets}

The dense deployment of HetNets helping to boost the capacity can can also result in a significant increase in energy consumption. For instance, the small cells deployed on enode $\mathrm{B}(\mathrm{enB})$ macro cells are shown in Fig. 2.1. We can see that there are no associated users in the $\mathrm{S} 3$ and M6 and these cells can be turned OFF in order to save energy. In some scenarios there might be few users associated in the cells and it make sense to handover the users to neighbouring base station, before turning them OFF. As a result, this leads to unnecessary handovers, handover failures (HF), throughput degradation at users due to large distance from the serving BS and also user even might experience outage. So there is a trade off between energy saving and user quality of service (QoS) enhancement. This calls for researchers to develop effective techniques that can reduce the network energy consumption without causing critical performance degradation in QoS.

According to China Mobile, the base stations (BSs) consume $72 \%$ of the total power consumption in cellular networks [36], which will be further increased with the additional deployment of the small cells. Therefore, network operators are seeking use of efficient BS power management techniques to reduce their operational expenditures. One approach is to introduce discontinuous transmission (DTX) on a BS when it is not serving any users as mentioned in [37]. In DTX, the cells are configured with almost blank subframes called multicast broadcast single frequency network for the efficient energy operation in LTE. Another approach is to turn off the BSs when there are no users communicating with them or when they are under-utilized [19, 38-45].

Centralized/distributed switching algorithms were proposed in [38-44] to turn off the BSs, and the associated users are handed over to the neighboring BSs, which 


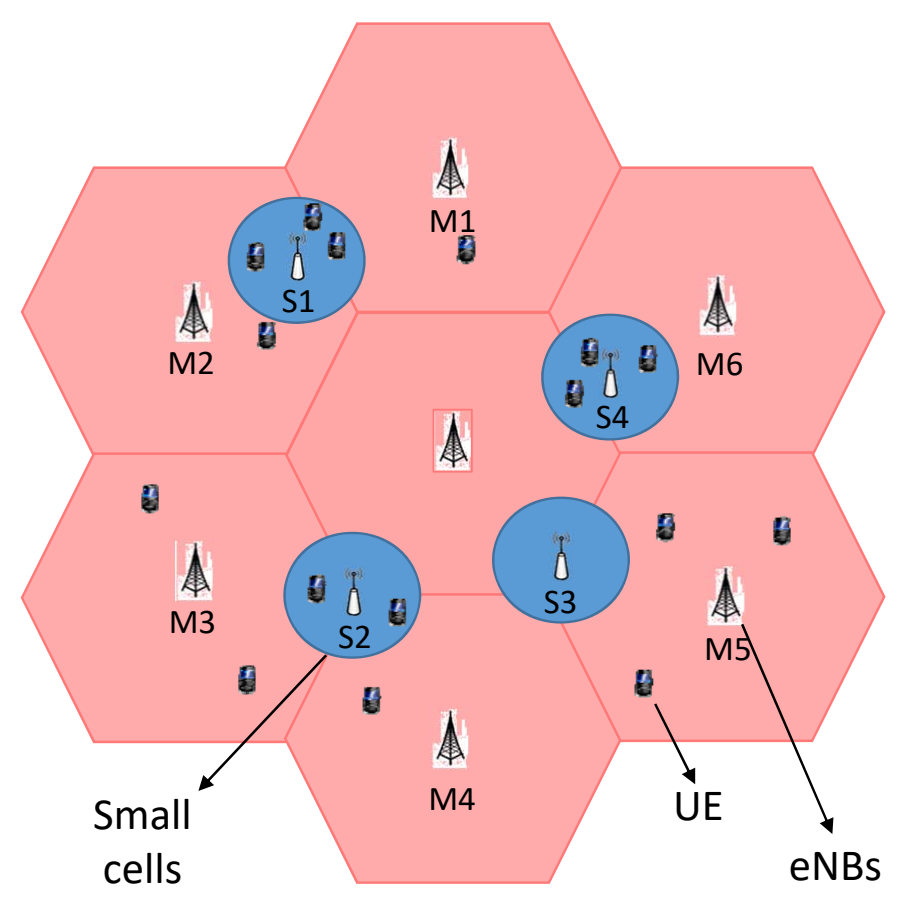

Figure 2.1: ON/OFF energy saving technique.

yields the significant savings in the energy expenditure for the cellular network operators $[46,47]$. The BSs can also adjust their transmission power, and antenna tilt angles according to the users' traffic load instead of shutting down completely [19,45,48-50]. In [51] a game theoretic framework was proposed where small base stations are able to autonomously adjust their transmission power without the need of a centralized controller. There is always a tradeoff between achieving energy efficiency and satisfying users' QoS constraints and the performance of centralized and distributed algorithms were analyzed with users' outage in $[49,52]$ and rest of other related works were summarized in $[53,54]$. However, these works did not explicitly account for the mobility of users in HetNet.

The mobility aspects of the energy efficiency is challenging due to the large number of network parameters involved during the mobility management of modern cellular networks, solving of a complex optimization problem that involves metrics such as energy efficiency, handover performance, and throughput can be intractable. In the 
ON/OFF switching setting, there are unnecessary handovers due to the mobility of the users and also additional user load bought by the switched off BS on the neighboring BS. As a result, there is a significant increase in the signaling load on the network. The authors in [55] aim to balance between the user association with the small cells and its power consumption through game theoretic framework and showed that signaling load can be reduced. Nevertheless, the handover scheme proposed in [55] did not account for the user speed and it is not robust to the handle the imprecise nature of the handover parameters in practical wireless cellular networks.

The observed/measured parameters such as the link quality, cell load, and user velocity, among others, may be imprecise and subject to uncertainties, introducing high complexity with limited benefit. Fuzzy logic approach seems suitable to handle this imprecision of the practical wireless cellular networks. The concept of fuzzy sets was proposed by Zadeh which maps the set elements to a membership function which indicates the degree of truth belonging to the set. This helps to express the imprecision, vagueness etc., in the real wireless cellular networks which cannot be easily studied. The authors in $[56,57]$ showed that incorporating fuzzy logic in the learning systems showed improved performance and was reliable in extremely noisy environments. Additionally, fuzzy logic framework allows the usage of human knowledge in the form of if-then inference rules. In [58], rule table was provided to reduce the ping-pong effects in an LTE network. The human based rules in fuzzy logic may not be optimal and requires the optimization techniques. The adaptive network fuzzy inference system proposed in $[59,60]$ used neural network approach to simplify the if-then rules of the fuzzy inference system and in [61,62], the inference rules of the fuzzy logic controller were refined using learning techniques to minimize the signaling load. The handover scheme in [61] considers only signal strength metric for the handover decision which can lead to high signaling overhead in the case of users traveling 
with high velocity in a densely deployed HetNet. Therefore context-aware handover scheme which considers multiple attributes (velocity [63,64], signal strength, QoS etc.), are necessary to minimize handovers and ensure seamless service to the UEs.

In this research work $[65,66]$, we introduce a fuzzy logic based game theoretic approach in order to have simplified optimization problem using the membership functions together with a game theoretic approach, for dynamically placing cells into sleep mode while also considering throughput and handover performance. We aim to optimize the fuzzy rules to obtain ideal transmission BS power levels for serving the UEs. Furthermore, a context-aware fuzzy handover scheme is proposed to minimize the unnecessary frequent handovers caused due to the dynamic power level switching of the BS. The fuzzy handover scheme consists of two modules: 1) handover decision and 2) target BS selection. For the handover decision, we use fuzzy inference system to check for the handover condition considering multiple user context parameters such as velocity, signal to interference plus noise ratio (SINR), throughput and BS load. Further, the fuzzy technique for order of preference by similarity to ideal solution (FTOPSIS) ranking method $[67,68]$ is used to select best BS during the target BS selection stage of the handover process.

\subsection{Experimental Study on Mobility Performance in Real LTE scenarios using SDR}

Drive test are the important practices performed by the operators to discover problems related to coverage and mobility performance of the network. Field trial were performed in order to investigate user QoS and handover performance in the real LTE [69-72] and test bed LTE advanced networks [73]. In [74] the post processing is performed on the measurement campaign at pedestrian walking speed to study 
the channel effects in Universal Mobile Telecommunications System (UMTS). There are many open source tools [75-77] to monitor and analyze the LTE signals. In [78], the recorded measurements were processed offline to decode master information block (MIB) and obtain system information like bandwidth and system frame number.

In this research work, post processing of drive test measurements is performed and base station coverage is evaluated. The impact of user velocity on mobility performance is investigated in highway and city scenarios. 


\section{CHAPTER 3}

\section{HANDOVER FAILURE ANALYSIS IN HETEROGENEOUS NETWORKS}

The mobility management is a challenging task. In any cellular networks, handover is the main process that supports seamless connectivity of UEs among base stations (BSs). In the Fig. 3.1 we can see that due to increased number of BSs in a HetNet, it is more challenging to support seamless mobility of UEs where handovers may fail more frequently [7].

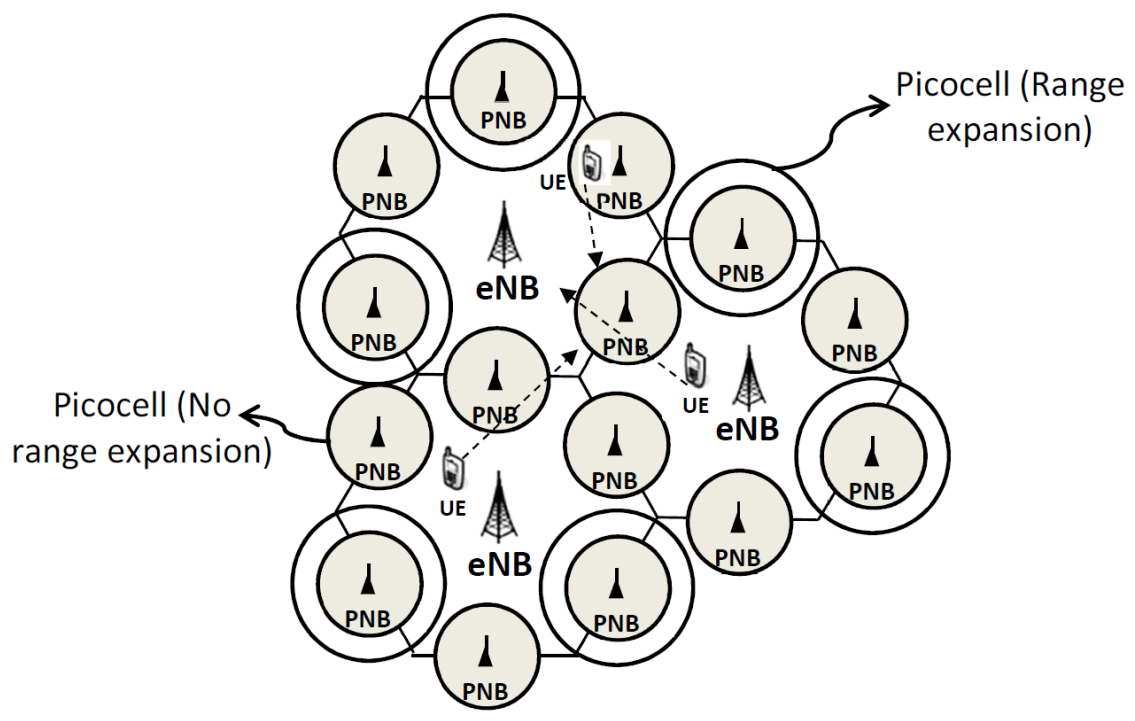

Figure 3.1: Signalling overhead problem in the dense HetNet scenario.

In the case of present Long Term Evolution (LTE) systems, signal measurements obtained at a UE from the neighboring BSs are reported by the UE to its serving BS to support handover decisions. In LTE HetNets, due to the small cell sizes, such measurement reporting by the UE may not be finalized sufficiently quickly, and this might result in severe handover failure (HF) problems for the high velocity UEs. Therefore it is important to study how measurement related parameters affect the handover performance in LTE HetNets. In the following section we study the handover process and measurements Procedure in LTE HetNets. 


\subsection{Review of the Handover Process in LTE}

The key steps of a typical handover process in a HetNet scenario are illustrated in Fig. $3.2[79,80]$. In LTE, UEs perform reference signal received power (RSRP) measurements to assess the proximity of neighbouring cells [81], and handover decisions are made based on these measurements. An example for the downlink (DL) RSRP measurement profile of a macrocell and a picocell, measured by a mobile UE, are shown in Fig. 3.2. Once the measurements are performed, the UE checks for the handover event entry condition, e.g., when the signal strength $P_{\mathrm{p}}$ from a target cell (e.g., a picocell) is larger than the signal strength from the serving cell (e.g., a macrocell) $P_{\mathrm{m}}$ plus a hysteresis threshold (step-1). When this condition is satisfied for the first time, the UE waits for a duration of TTT, before sending a measurement report to its serving cell (step-2) to initiate the actual handover.

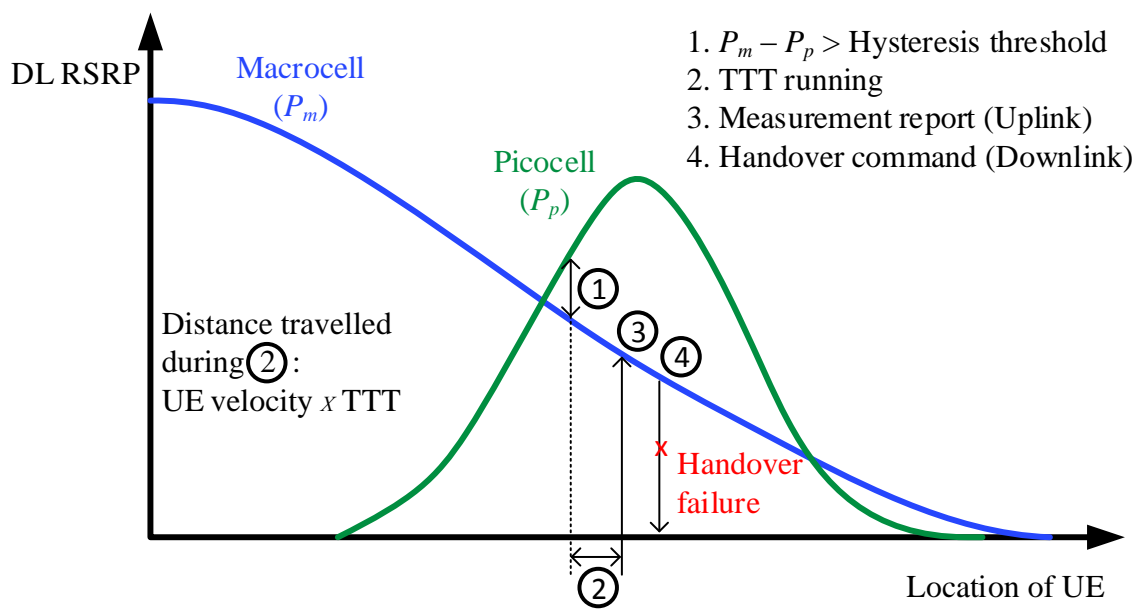

Figure 3.2: Handover failure problem in HetNets due to small cell size.

The use of a TTT is critical to ensure that ping-pongs (successive and unnecessary handovers among neighboring cells generated due to fluctuations in the link qualities from different cells), are minimized. If the handover event entry condition is still satisfied after the TTT, the UE sends a measurement report to its serving BS (step- 
3), which then communicates with the target BS. If both BSs have an agreement and the handover is to be performed, the serving BS sends a handover command to the UE to indicate when it is should connect to the target BS (step-4). The handover process is finalized when the UE sends a handover complete to the target BS, indicating that the handover process was completed successfully (step-5) [82].

\subsubsection{Handover Failures and Ping-Pongs}

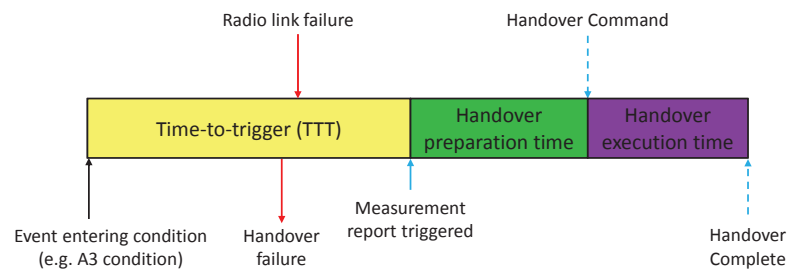

(a) HF due to RLF.

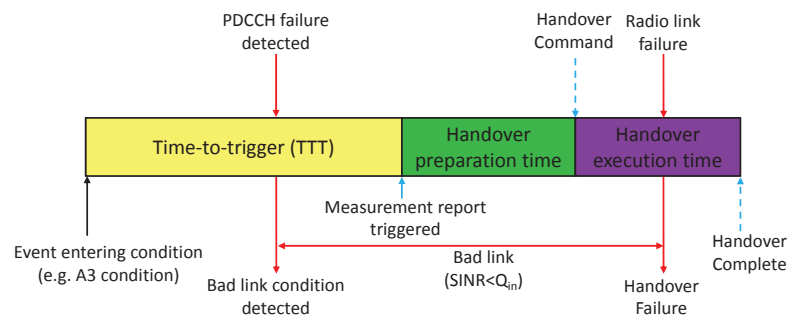

(b) HF due to PDCCH failure detection.

Figure 3.3: Modeling of the HF based on 3GPP mobility management studies for HetNets [83].

Based on the 3GPP specifications [7,83], a handover failure can be declared at a UE if one of the following three conditions is met:

1. A radio link failure happens during the time between satisfying a measurement triggering even (such as the A3 event [83]) condition and receiving handover command, shown in Fig. 3.3(a). 
2. A T310 timer is triggered due to detection of physical downlink control channel (PDCCH) failure, and still running when a handover command is sent, shown in Fig. 3.3(b).

3. The UE wideband signal to interference plus noise ratio (SINR) is lower than a threshold $Q_{\text {out }}$ (in $\mathrm{dB}$ ) when a handover complete message is sent, shown in Fig. 3.3(b).

A PDCCH failure is detected when a UE's wideband SINR falls to $Q_{\text {out }}$ (in dB) and the link is considered to be recovered when the SINR reaches $Q_{\text {in }}$ (in dB).

Ping-Pong (PP) is one of the factors which degrades network performance due to additional overhead. PPs are considered as unnecessary handovers (HOs) and it depends on time-of-stay of the UE. It starts when the UE sends a HO complete message to a cell, and ends when the UE sends a HO complete message to another cell. If a UE has a time-of-stay less than threshold $T_{\mathrm{pp}}$ then the $\mathrm{HO}$ that terminates this time-of-stay is considered as an unnecessary HO. An unnecessary HO is consider as a PP if the prior-to-source and target cell is the same cell, where the prior-to-source cell is the cell to which the UE was connected before handing over the source cell. The $T_{\mathrm{pp}}$ is set to $1 \mathrm{~s}$ specified in [83] and PP rate is defined as the ratio of number of PPs to total number of successful HOs (excluding HFs).

\subsubsection{Handover Measurements Procedure in LTE.}

Different measurements obtained at a UE during a handover process are summarized in Fig. 3.4 [84]. As shown in Fig. 3.4(a), the RSRP measurements $P_{\mathrm{m}}$ and $P_{\mathrm{p}}$ at a UE are obtained after a filtering process in order to mitigate the effects of channel fluctuations. The filtering is performed at two levels, Layer-1 (L1) and Layer-3 (L3). Initially, the UE obtains an RSRP sample by linear averaging over the power contribution of all reference symbols carrying the common reference signal within one 


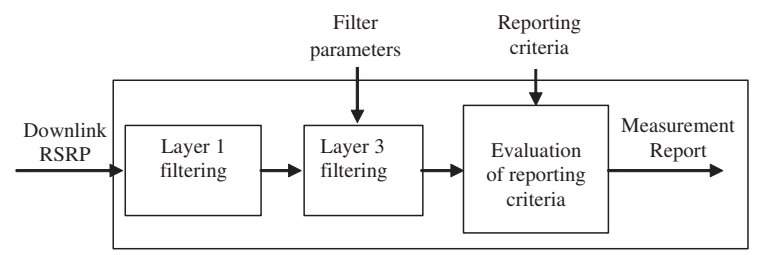

(a) Handover measurement model specified in [84].
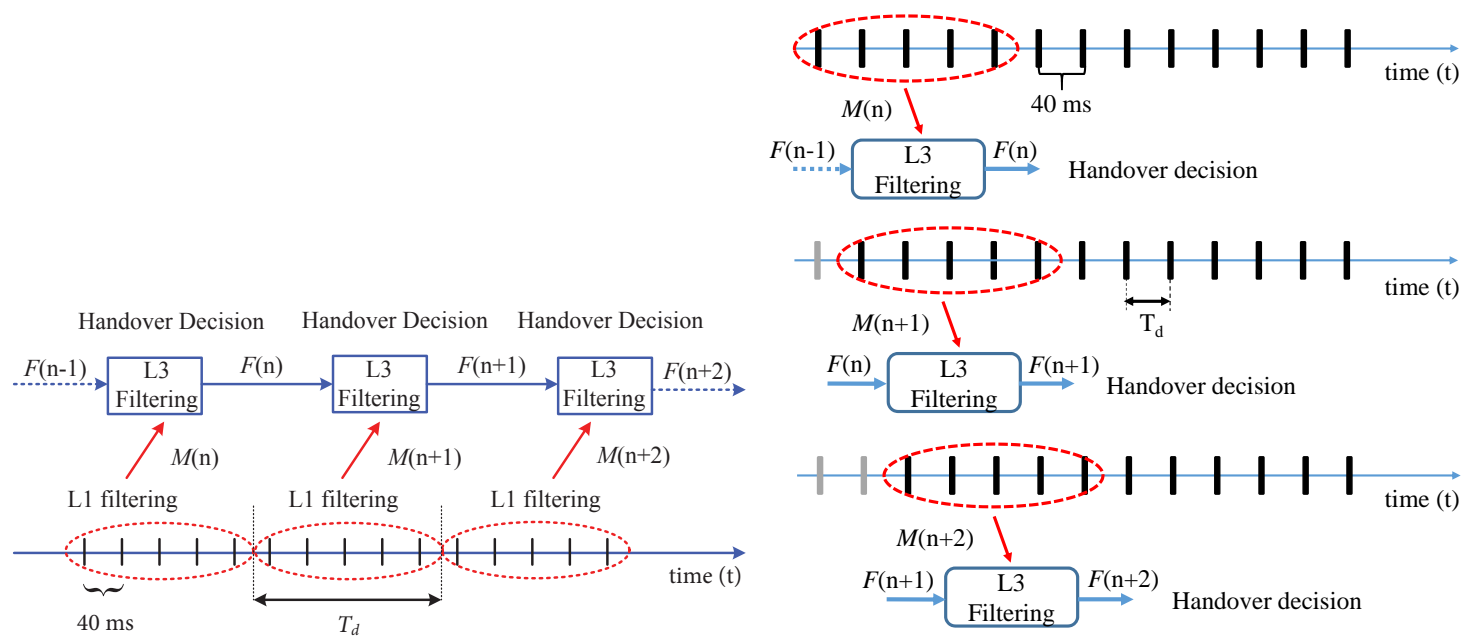

(b) Processing of the RSRP measurements through L1 and (c) L3 filtering at a UE as in [7].

Processing of the RSRP measurements through L1 and L3 filtering at a UE, filtering at a UE, using a sliding window as per $3 \mathrm{GPP}$ specifications [83].

Figure 3.4: Handover measurement performed by the UE through two different L1 filtering approaches.

subframe (i.e., $1 \mathrm{~ms}$ ). The measurement bandwidth is of at least six physical resource blocks. Subsequently, L3 filtering further averages the L1 samples using a first-order infinite impulse response (IIR) filter. The 3GPP specifications consider a sliding window approach for obtaining the L1 samples, while in our earlier work of $[7,32,34,35]$, we deviated from 3GPP specifications without using a sliding window, which provides a suboptimal approach. For comparison purposes and to acknowledge the difference, we will describe both scenarios in this research work (see Fig. 3.4(b) and Fig. 3.4(c)), and present related results in Section 3.5.1 to compare their performance.

First, according to the considered handover measurement process in the earlier work $[7,32,34,35]$, the UE performs the L1 filtering by obtaining an RSRP sample every $40 \mathrm{~ms}$, and performs a linear averaging over 5 successive RSRP samples as shown 
in Fig. 3.4(b). As a result, the L1 filtering performs averaging over every $200 \mathrm{~ms}$ to obtain an L1 sample, $M(n)$, given by [7]

$$
M(n)=\frac{1}{5} \sum_{\kappa=0}^{4} R S R P_{\mathrm{L} 1}(5 n-\kappa),
$$

where $n$ is the discrete time index of the RSRP sample, $R S R P_{\mathrm{L} 1}$ is the RSRP sample measured every $40 \mathrm{~ms}$ by the UE, and $\kappa$ is the delay index of the filter. As shown in Fig. 3.4(b), no sliding window is considered to obtain subsequent L1 samples. The UE further averages the L1 samples through a first-order IIR (L3) filter, given by

$$
F(n)=(1-a) F(n-1)+a 10 \log _{10}[M(n)]
$$

where $a=\left(\frac{1}{2}\right)^{\frac{k}{4}}$ is the forgetting factor and $k$ is the L3 filter coefficient [82]. Finally, the UE periodically checks whether the resulting L3 sample meets the handover entry condition every $T_{\mathrm{d}}$ seconds, where $T_{\mathrm{d}}$ refers to L3 sampling period (e.g., $200 \mathrm{~ms}$ in 3GPP LTE [14]). If the handover entry condition is satisfied, then rest of the handover steps may follow as described previously.

In the second approach, the handover measurement process stated in 3GPP [83] specifies that the UE performs the L1 filtering more frequently (e.g., $40 \mathrm{~ms}$ ) by averaging using a sliding window size of $200 \mathrm{~ms}$, as outlined in Fig. 3.4(c). This means that a new L1 sample is obtained by averaging over previous RSRP samples within the sliding window. If we consider $T_{\mathrm{d}}$ as the filter sampling period then the number of RSRP samples within the sliding window is given by the L1 filter sliding window size $N_{L_{1}}=\frac{200 \times 10^{-3}}{T_{\mathrm{d}}}$ and the samples outside the sliding window will be discarded. Therefore $M(n)$ can be expressed as

$$
M(n)=\frac{1}{N_{L_{1}}} \sum_{\kappa=0}^{N_{L_{1}}-1} R S R P_{\mathrm{L} 1}(n-\kappa),
$$

where $n$ is the discrete time index of the RSRP sample, $R S R P_{\mathrm{L} 1}$ is the RSRP sample measured every $T_{\mathrm{d}}$ seconds by the UE, and $\kappa$ is the delay index of the filter. 
The UE further averages the L1 samples through a first-order IIR (L3) filter as follows

$$
F(n)=(1-a) F(n-1)+a 10 \log _{10}[M(n)]
$$

where $a=\left(\frac{1}{2}\right)^{\frac{k}{4}}$ is the forgetting factor and $k$ is the L3 filter coefficient scaled according to the sampling period of the L3 filter [82]. According to 3GPP TS 25.302 specification [84], the L3 filter sampling rate should have the same rate as its inputs i.e., the L1 filter sampling rate. Therefore $T_{\mathrm{d}}$ can be expressed as the identical sampling period for both L1 and L3 filters. Finally, the UE periodically checks whether the resulting L3 sample meets the handover entry condition every $T_{\mathrm{d}}$ seconds. If the handover entry condition is satisfied, then rest of the handover steps may follow as described previously.

\subsection{Geometric Model for Handover Performance Analysis}

In order to evaluate the handover performance in HetNets, a standard hotspot model with specific simulation scenarios and parameters was proposed in the $3 \mathrm{GPP}$ study item [83]. This hotspot model is based on a bouncing (hotspot) circle concentric within the picocell, whose radius is assumed to be $200 \mathrm{~m}$. Note that this radius of the bouncing circle is different (larger) than the radius of the picocell. The starting UE position is chosen randomly on the bouncing circle, and the UE follows a linear trajectory towards the picocell BS with a random angle. The UE does not change its direction until it hits again the bouncing circle, and when it does it bounces back with a random angle, as shown in Fig. 3.5. With such a model, theoretical analysis of the HF probabilities is challenging, due to the complexity of modeling the statistics of a UE's sojourn time with in a picocell. Instead, we propose a simpler geometric model for the handover performance analysis. 


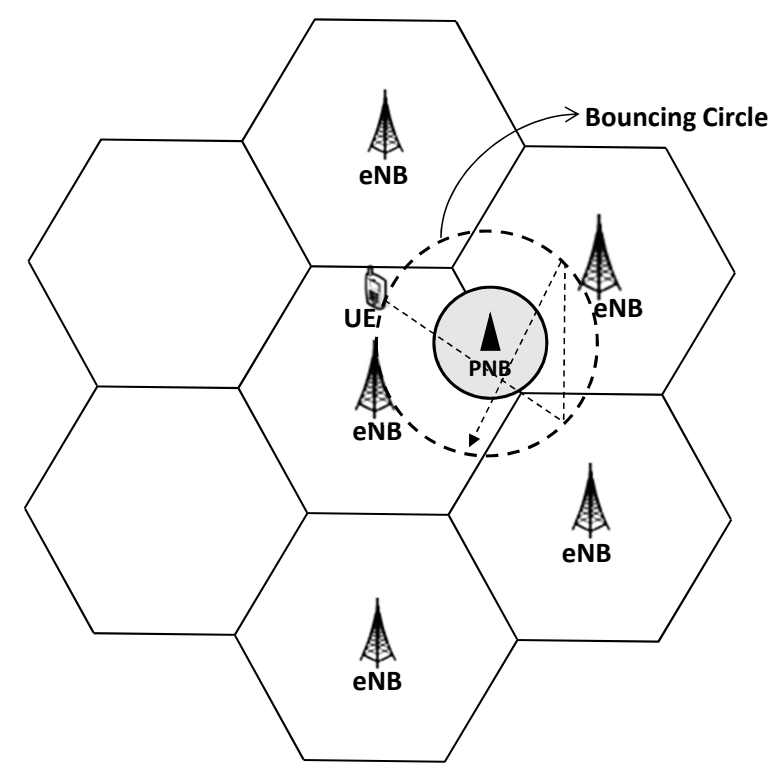

Figure 3.5: The bouncing ring UE mobility model from [83].

In order to simplify the handover model shown in Fig. 3.5, we initially consider the handover metrics of a single user in the absence of fading, and develop a new framework to facilitate closed form analysis of HF probabilities. Later, using this framework as a reference, we extend our analysis of HF probabilities into the scenario where there is channel fading.

The simulator is designed according to the 3GPP specifications in [83]. The handover measurement procedure is implemented according to the description shown in Section II.B. Handover failure condition is summarized in Fig. 2, and it is detected using radio link monitoring process described in [83]. In particular, the wideband SINR measurements of the target cell are performed by the UE at every $10 \mathrm{~ms}$ and then they are filtered through different sliding windows using L1/L3 filters. The filtered measurements are then compared with $Q_{\text {out }}$ and $Q_{\text {in }}$ using $200 \mathrm{~ms}$ and $100 \mathrm{~ms}$ sliding windows, respectively. The corresponding handover trigger (red dots, after step-1 in Fig. 1) and handover failure locations (blue cross) are shown in Fig. 5. The scattered ring of handover trigger locations is a result of the discrete measurement process carried out at UE. These handover trigger locations extend inwards from the ideal 
coverage area of a pico BS (PBS), since a UE may delay initiation of the TTT due to the filtered RSRP measurements being available only with $T_{\mathrm{d}} \mathrm{s}$ intervals. Fig. 3.6 also shows that if we neglect the impact of channel fading as well as sectorized cell structure, the HF locations (the blue cross signs where the wideband SINR becomes lower than a threshold) can be well approximated by a circle.

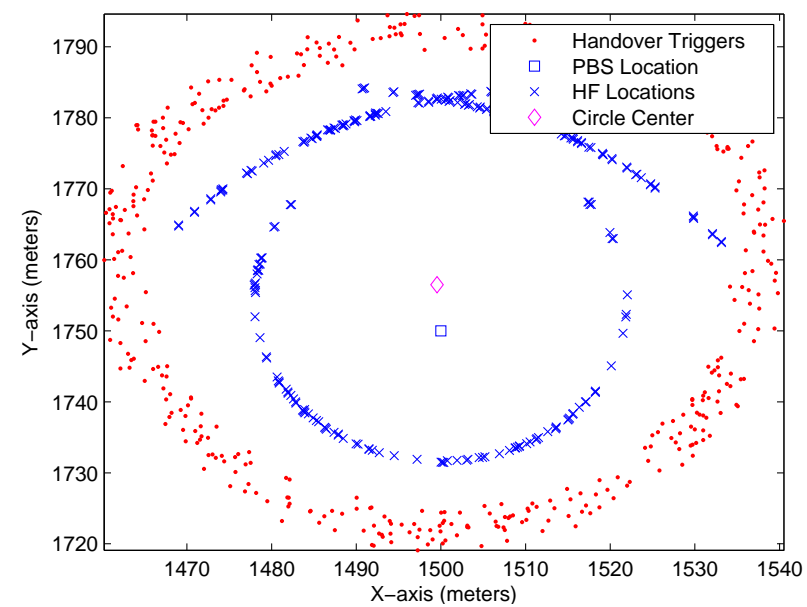

Figure 3.6: Handover trigger and HF locations in an example picocell using a 3GPPcompliant simulator. The MBS is located at $(1500,1500) \mathrm{m}$ and has three sectors $\left(T_{\mathrm{d}}=200 \mathrm{~ms}\right)[32]$.

Based on Fig. 3.6, we model the picocell coverage are and HF locations geometrically as concentric circles with radius $R$ and $r_{\mathrm{m}}$, respectively, as shown in Fig. 3.7. Note that the HF locations shown in Fig. 3.6 are for macro to pico HFs and in the same way it is reasonable to approximate pico to macro HF locations as another concentric circle with $r_{\mathrm{p}}$, as shown in Fig. 3.7. In such figure, $v$ denotes UE velocity, $\theta$ is the angle of UE trajectory with respect to horizontal axis and $T_{\mathrm{m}}, T_{\mathrm{p}}$ are the TTT duration for MUE and PUE respectively.

In the following subsection, we incorporate the discrete measurements process in the presented geometric model by proposing a standard distribution that fits to the handover trigger locations. Thereafter, we use this standard distribution to model 


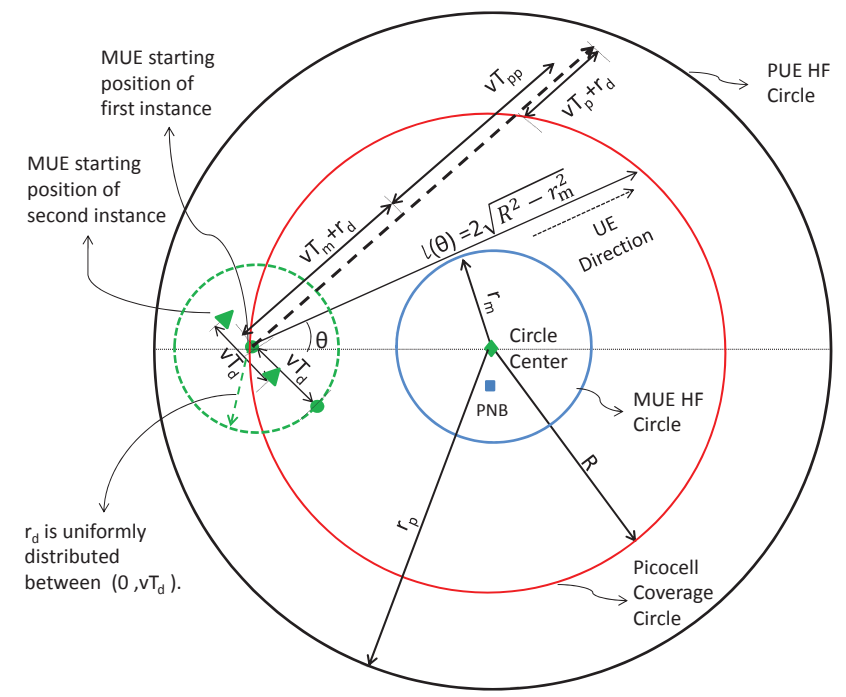

Figure 3.7: Geometric handover model to analyze HFs for MUEs and PUEs, and considers the effects of L3 filtering. The parameter $l(\theta)$ refers to the length of the segment for the UE trajectory, for which the segment becomes a chord of the picocell coverage circle.

the UE's sojourn time in order to facilitate the theoretical analysis of macro-cell UE (MUE) and pico-cell UE (PUE) HF.

\subsubsection{Modeling the Handover Trigger Locations}

To model the statistics of the handover trigger locations, which are offset due to L1/L3 filtering, we examine the distance of each handover trigger location shown in Fig. 3.6 to the ideal picocell coverage boundary, which we define as handover offset distance. In this research work, we model the distribution of the handover offset distance using a uniform distribution for the scenario with no fading, and using a custom distribution for the scenario with fading (to be studied in Section 3.4).

In the following we describe how we incorporate the discrete measurement process in the geometric model presented Fig. 3.7. Let us consider a UE traveling in a straight line with a velocity $v$. Due to the discrete measurements performed by the UE every $T_{\mathrm{d}} \mathrm{s}$, the UE checks whether the handover condition is satisfied at integer multiples of $T_{\mathrm{d}}$. Therefore, when the UE crosses the picocell coverage circle, TTT may not 
be immediately initiated. The UE may need to wait for a fraction of time until $T_{\mathrm{d}}$ expires, whose duration depends on where the UE processed the measurement before crossing into the picocell coverage area. Let us consider the different starting instance of UE crossing the picocell coverage area shown in Fig. 3.7. In the first instance, the UE starts as an MUE at the green dot and travels a distance $v T_{\mathrm{d}}$ entering the picocell coverage circle at some point. The TTT is triggered after distance $v T_{\mathrm{d}}$, at the second green dot. In the second instance, the UE starts earlier than the first instance as an MUE at the green triangle and also travels a distance $v T_{\mathrm{d}}$ entering the picocell coverage circle at some point. The TTT is also triggered after distance $v T_{\mathrm{d}}$, at the second green triangle. Note that the location where the TTT would be triggered after L1/L3 filtering in the second instance is closer to the ideal picocell coverage boundary than the first instance. In this research work, we model the distance between the triggering location of the TTT and the picocell coverage circle as a random variable, and we denote this distance as $r_{\mathrm{d}}$. If we consider all possible instances, we can assume that the distance from the handover trigger locations to the cell edge reference point, $r_{\mathrm{d}}$, is uniformly distributed within $v T_{\mathrm{d}}$, i.e. $r_{\mathrm{d}} \sim \mathcal{U}\left[0, v T_{\mathrm{d}}\right)$.

To verify our model, we check the histogram of handover offset distance $r_{\mathrm{d}}$ generated by our 3GPP-compliant simulator. Based on the assumptions shown in Fig. 3.5 and handover parameters shown in Table 3.1, the handover trigger locations are generated for the following three cases:

1. Case-1: Without shadowing and fast-fading.

2. Case-2: With shadowing but without fast-fading.

3. Case-3: With shadowing and fast-fading.

The handover offset distance histograms for Case-1 is shown in Fig. 3.8. We can see that for a $60 \mathrm{~km} / \mathrm{hr} \mathrm{UE}$, and for the scenario of $T_{\mathrm{d}}=200 \mathrm{~ms}$ adopted in 3GPP LTE, the handover offset distance histogram can be reasonably modeled using 
Table 3.1: Handover parameter sets.

\begin{tabular}{|l|l|l|l|l|}
\hline Profile & Set-1 & Set-2 & Set-3 & Set-4 \\
\hline TTT (ms) & 480 & 160 & 80 & 40 \\
\hline L3 filter coefficient $(k)$ & 4 & 1 & 1 & 0 \\
\hline
\end{tabular}

a uniform distribution. Modeling the handover process in the fading scenario follows from Case-1, and is achieved by finding the handover offset distance histograms for the trigger locations in the Case-2 and Case-3, which is studied in Section 3.4.

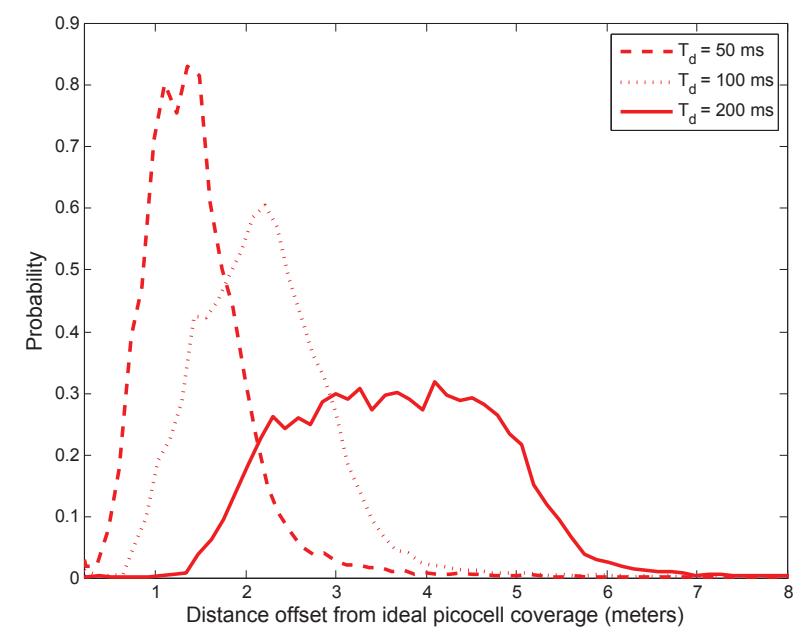

Figure 3.8: Handover offset distance histograms for $v=60 \mathrm{~km} / \mathrm{hr}$ in Case- 1 . For the scenario of $T_{\mathrm{d}}=200 \mathrm{~ms}$ adopted in 3GPP LTE, the handover offset distance histogram can be reasonably modeled using a uniform distribution.

\subsubsection{Modeling the UEs' Sojourn Times}

The sojourn time estimated in a picocell may be different when using the geometric model in Fig. 3.7 or when using the bouncing ring model of Fig. 3.5. In order to justify the use of our geometric model in Fig. 3.7 to model the scenario in Fig. 3.5, we consider the Bertrand's Paradox [85] and the three probability density functions described therein. We will show that one of these probability density functions, based on a geometric model like ours, well matches the behaviour of the bouncing ring model, thus validating our modelling. 


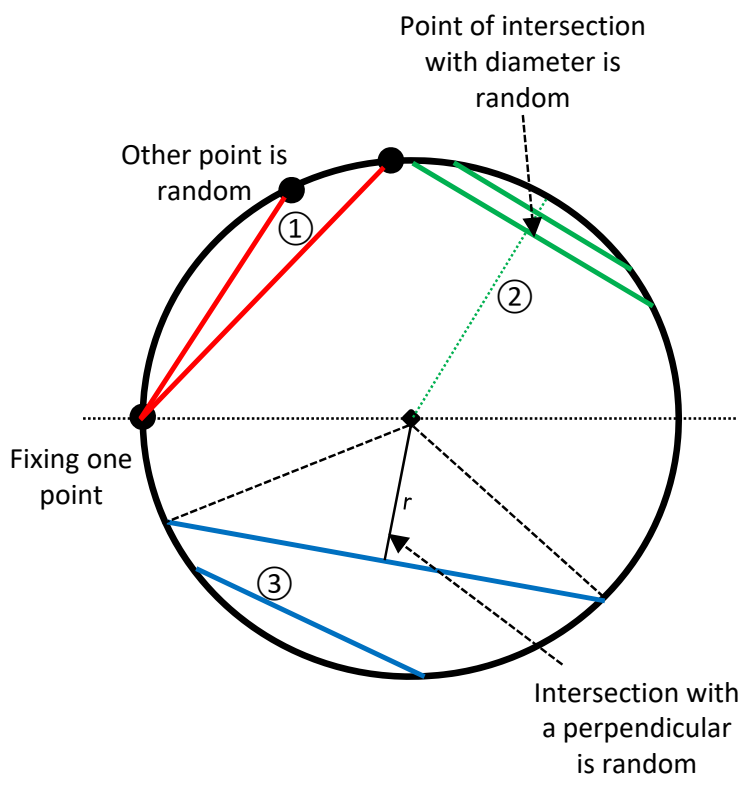

Figure 3.9: Different interpretations of randomness of a chord in a circle

The Bertrand's Paradox studies the probability that a random chord of a circle with a radius $R$ is larger than a threshold. In essence, this probability leads us to the statistics of the sojourn time in a given picocell. Due to the different interpretation of randomness of a chord in a circle shown in Fig. 3.9, there are three different models for the PDF of the chord length.

Model 1: When we choose randomly two points on a circle and draw the chord joining them, without loss of generality, we may position ourselves at one of them and examine the relative location of the other points. If angle $\theta$ is uniformly distributed between $\left[-\frac{\pi}{2}, \frac{\pi}{2}\right]$, the PDF of the chord length $l$ is then given by:

$$
f_{1}(l)=\frac{2}{\pi \sqrt{4 R^{2}-l^{2}}}
$$

Note that this interpretation corresponds to the model used in Fig. 3.7.

Model 2: If we choose a chord whose direction is fixed and perpendicular to a given diameter of the circle, then we assume that the point of intersection of the chord with the diameter has a uniform distribution. Therefore we can assume that 
the perpendicular distance $r=\sqrt{R^{2}-\frac{d^{2}}{4}}$ from the chord to the center of the circle is uniformly distributed between $[0, R]$. The PDF of the chord length $l$ is then given by:

$$
f_{2}(l)=\frac{l}{2 R \sqrt{4 R^{2}-l^{2}}} .
$$

Model 3: A chord is uniquely determined by its midpoint, for which a perpendicular line extending from the circle center intersects with the chord. If this intersection point is uniformly distributed over the entire circle, the PDF of the chord length $l$ is then given by:

$$
f_{3}(l)=\frac{l}{2 R^{2}}
$$

In order to evaluate how closely the three approaches in the Bertrand's Paradox capture the picocell sojourn time in Fig. 3.5, we compare the PDFs of chord lengths for the three Bertrand's Paradox cases with the simulated chord length histogram for the bouncing ring model presented in Fig. 3.5. Considering $R=21.7 \mathrm{~m}$ and plotting the histograms of chord length overlayed with PDFs of all the three solutions in (3.5)(3.7), we obtain the results in Fig. 3.10. Model 1 shows a reasonable match with the simulated chord lengths, and thus we adopt the PDF given in (3.5) to mode sojourn time and drive our MUE and PUE HF analysis in both no fading and fading scenarios.

\subsection{Handover Failure Analysis without Fading}

Using the geometric model in Fig. 3.7 and the PDF of chord length in (3.5), in this section, we derive the HF probabilities for MUEs and PUEs considering L3 filtering and no fading. We consider that a UE checks the handover entry condition at every $T_{\mathrm{d}}$ sampling period of the L3 filter. When the handover condition is satisfied, then TTT of duration $T_{\mathrm{m}}$ is triggered. The $r_{\mathrm{d}}$ is a random variable accounting for discrete 


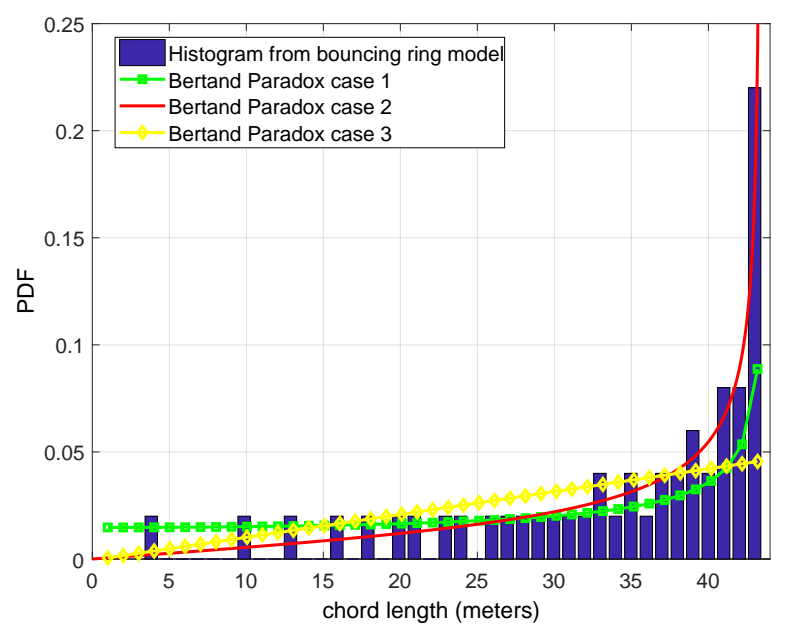

Figure 3.10: PDFs of the chord length for the three different approaches in Bertrand's Paradox, and the histogram of the chord length for the bouncing-ring simulations in Fig. 3.5.

measurement interval carried out in the UE through L3 filtering, and we assume that $r_{\mathrm{d}}$ is uniformly distributed between $\left[0, v T_{\mathrm{d}}\right]$, yielding the following PDF

$$
f\left(r_{\mathrm{d}}\right)= \begin{cases}\frac{1}{v T_{\mathrm{d}}} & 0 \leq r_{\mathrm{d}}<v T_{\mathrm{d}} \\ 0 & \text { otherwise }\end{cases}
$$

In other words, $r_{\mathrm{d}}$ models the random offset between the intersection of the UE trajectory with the ideal picocell coverage circle, and the location when the filtered measurements become available to the UE after entering the picocell's coverage area. In the following section, we derive the no handover (NHO) probability for MUEs and HF probabilities for both MUEs and PUEs, using Fig. 3.7 and equations (3.5) and (3.8).

\subsubsection{No Handover Probability for MUEs}

After TTT of duration $T_{\mathrm{m}}$ is triggered, the MUE does not make a handover if it leaves the picocell coverage circle before the end of $T_{\mathrm{m}}$. The total distance traveled by the MUE after its last L3 sample and before triggering a handover is equal to $v T_{\mathrm{m}}+r_{\mathrm{d}}$. Depending on the picocell coverage area and the value of $v T_{\mathrm{m}}$ relative to $r_{\mathrm{d}}$, NHO probability for a given UE can be analyzed for three different cases. 
1) $v T_{\mathrm{m}}<2 \sqrt{R^{2}-r_{\mathrm{m}}^{2}}-v T_{\mathrm{d}}$ : According to the Bertrand's Paradox model 1 shown in Section 3.2.2, the MUE trajectory angle $\theta$ is uniformly distributed and can take any value between $\left[-\frac{\pi}{2}, \frac{\pi}{2}\right]$. If we start tracing the line segment of distance $v T_{\mathrm{m}}+r_{\mathrm{d}}$ on the picocell boundary shown in Fig. 3.7 with angle values ranging from 0 to $\frac{\pi}{2}$, there will be an angle $\theta$ for which the line segment will be a chord of the picocell coverage circle. Further tracing with increasing angle values will make the final point of the MUE trajectory to be located outside the picocell coverage circle; this implies that the UE will go outside of the picocell coverage area while the TTT timer is running, and hence there will be no handover to the picocell. As a result, we can obtain the NHO probability by finding the probability that $\theta$ is smaller than the chord length $l(\theta)=v T_{\mathrm{m}} \cos (\theta)$, which is expressed as

$$
\mathcal{P}_{\mathrm{NHO}}=\mathbb{P}\left(l(\theta)<v T_{\mathrm{m}}+r_{\mathrm{d}}\right)=\int_{0}^{v T_{\mathrm{m}}} \frac{2}{\pi \sqrt{4 R^{2}-l^{2}}} \mathrm{~d} l+\int_{0}^{v T_{\mathrm{d}}} \frac{1}{v T_{\mathrm{d}}} \int_{v T_{\mathrm{m}}}^{v T_{\mathrm{m}}+r_{\mathrm{d}}} \frac{2}{\pi \sqrt{4 R^{2}-l^{2}}} \mathrm{~d} r \mathrm{~d} r_{\mathrm{d}}
$$

Since the random variable $r_{\mathrm{d}}$ and $\theta$ are independent, we can multiply the PDFs presented in (3.5) and (3.8), as shown in (3.9), and the final closed form NHO probability can be expressed as

$$
\begin{aligned}
\mathcal{P}_{\mathrm{NHO}}=\mathbb{P}\left(l(\theta)<v T_{\mathrm{m}}+r_{\mathrm{d}}\right) & =\frac{2}{\pi v T_{\mathrm{d}}}\left[\sqrt{4 R^{2}-\left(v T_{\mathrm{m}}+v T_{\mathrm{d}}\right)^{2}}+\left(v T_{\mathrm{m}}+v T_{\mathrm{d}}\right) \tilde{T}_{\mathrm{d}}\right. \\
& \left.-\sqrt{4 R^{2}-\left(v T_{\mathrm{m}}\right)^{2}}-v T_{\mathrm{m}} \tan ^{-1}\left(\frac{v T_{\mathrm{m}}}{\sqrt{4 R^{2}-\left(v T_{\mathrm{m}}\right)^{2}}}\right)\right],
\end{aligned}
$$

where,

$$
\tilde{T}_{\mathrm{d}}=\tan ^{-1}\left(\frac{v T_{\mathrm{m}}+v T_{\mathrm{d}}}{\sqrt{4 R^{2}-\left(v T_{\mathrm{m}}+v T_{\mathrm{d}}\right)^{2}}}\right)
$$


2) $v T_{\mathrm{m}} \geq 2 \sqrt{R^{2}-r_{\mathrm{m}}^{2}}-v T_{\mathrm{d}}$ : If the distance traveled by the MUE by the end of the TTT duration is greater than or equal to the chord length $2 \sqrt{R^{2}-r_{\mathrm{m}}^{2}}$ shown in Fig. 3.7, the MUE will intersect with the MUE HF circle for some values of $\theta$, incurring HF. Then, to obtain the NHO probability, we have to subtract form the NHO probability computed in case 1) the mentioned MUE HF probability, which yields

$$
\begin{aligned}
\mathcal{P}_{\mathrm{NHO}} & =\mathbb{P}\left(l(\theta)<v T_{\mathrm{m}}+r_{\mathrm{d}} \mid r_{\mathrm{d}} \leq 2 \sqrt{R^{2}-r_{\mathrm{m}}^{2}}-v T_{\mathrm{m}}\right) \\
& =\int_{0}^{v T_{\mathrm{m}}} \frac{2}{\pi \sqrt{4 R^{2}-l^{2}}} \mathrm{~d} l+\int_{0}^{v T_{\mathrm{d}}} \frac{1}{v T_{\mathrm{d}}} \int_{v T_{\mathrm{m}}}^{v T_{\mathrm{m}}+r_{\mathrm{d}}} \frac{2}{\pi \sqrt{4 R^{2}-l^{2}}} \mathrm{~d} r \mathrm{~d} r_{\mathrm{d}}-\int_{2 \sqrt{R^{2}-r_{\mathrm{m}}^{2}}-v T_{\mathrm{m}}}^{v T_{\mathrm{d}}} \frac{1}{v T_{\mathrm{d}}} \int_{v T_{\mathrm{m}}+r_{\mathrm{d}}}^{v T_{\mathrm{m}}+v T_{\mathrm{d}}} \frac{2}{\pi \sqrt{4 R^{2}-l^{2}}} \mathrm{~d} r \mathrm{~d} r_{\mathrm{d}} .
\end{aligned}
$$

The limits of the random variable $r_{\mathrm{d}}$ in the third term represent the integration are which overlaps with the mentioned MUE HF circle. After solving the integral in (3.12) and using $\tilde{T}_{\mathrm{d}}$, we obtain

$$
\begin{aligned}
\mathcal{P}_{\mathrm{NHO}} & =\frac{2}{\pi v T_{\mathrm{d}}}\left[2 \sqrt{4 R^{2}-\left(v T_{\mathrm{m}}+v T_{\mathrm{d}}\right)^{2}}-\sqrt{4 R^{2}-\left(v T_{\mathrm{m}}\right)^{2}}-\left(2 \sqrt{R^{2}-r_{\mathrm{m}}^{2}}\right) \tan ^{-1}\left(\frac{\sqrt{R^{2}-r_{\mathrm{m}}^{2}}}{r_{\mathrm{m}}}\right)\right. \\
& \left.-v T_{\mathrm{m}} \tan ^{-1}\left(\frac{v T_{\mathrm{m}}}{\sqrt{4 R^{2}-\left(v T_{\mathrm{m}}\right)^{2}}}\right)-2 r_{\mathrm{m}}+\left(v T_{\mathrm{m}}+v T_{\mathrm{d}}+2 \sqrt{R^{2}-r_{\mathrm{m}}^{2}}\right) \tilde{T}_{\mathrm{d}}\right] .
\end{aligned}
$$

3) If the UE velocity is high, for which the product $v T_{\mathrm{m}}$ becomes greater than the chord length $2 \sqrt{R^{2}-r_{\mathrm{m}}^{2}}$, then the random variable $r_{\mathrm{d}}$ will have no effect, and the NHO probability will be constant. The reason for this is that if $\theta$ is larger than a threshold (for which the UE trajectory is tangent to the HF circle in Fig. 3.7), the UE will always be moving beyond the coverage area of the picocell before the TTT expires. Then, using (3.5), the NHO probability for this third scenario becomes 


$$
\mathcal{P}_{\mathrm{NHO}}=\mathbb{P}\left(l(\theta)<2 \sqrt{R^{2}-r_{\mathrm{m}}^{2}}\right)=\int_{0}^{2 \sqrt{R^{2}-r_{\mathrm{m}}^{2}}} \frac{2}{\pi \sqrt{4 R^{2}-l^{2}}} \mathrm{~d} l=\frac{2}{\pi} \tan ^{-1}\left(\frac{\sqrt{R^{2}-r_{\mathrm{m}}^{2}}}{r_{\mathrm{m}}}\right) .
$$

\subsubsection{HF Probability for MUEs}

When the MUE reaches the MUE HF circle before the TTT expires, there will be HF, and the MUE fails to connect with the picocell. This occurs when the MUE trajectory distance $v T_{\mathrm{m}}+r_{\mathrm{d}}$ is greater than the distance $d_{\mathrm{HF}, \mathrm{m}}\left(\theta, R, r_{\mathrm{m}}\right)$ for a given trajectory angle $\theta$. This distance refers to the total distance travelled by the UE from the ideal picocell coverage to the MUE HF circle, and it is given by

$$
d_{\mathrm{HF}, \mathrm{m}}\left(\theta, R, r_{\mathrm{m}}\right)=R \cos (\theta)-\sqrt{r_{\mathrm{m}}^{2}-R^{2} \sin ^{2}(\theta)}
$$

To obtain the MUE HF probability, we first evaluate the MUE HF condition $v T_{\mathrm{m}}+r_{\mathrm{d}}>d_{\mathrm{HF}, \mathrm{m}}\left(\theta, R, r_{\mathrm{m}}\right)$ in terms of $l(\theta)$. This is done to get the integrating limits for MUE HF probability, and corresponds to the condition for which the MUE will cross the HF circle before the TTT expires. Using (3.15) along with this condition, we can write

$$
v T_{\mathrm{m}}+r_{\mathrm{d}}>R \cos (\theta)-\sqrt{r_{\mathrm{m}}^{2}-R^{2} \sin ^{2}(\theta)}
$$

and using $l(\theta)=2 R \cos (\theta)$, we get $\theta=\cos ^{-1}\left(\frac{l(\theta)}{2 R}\right)$. Applying this this in (3.16), we obtain the MUE HF condition as a function of $l(\theta)$ as

$$
l(\theta)>\frac{R^{2}-r_{\mathrm{m}}^{2}}{v T_{\mathrm{m}}+r_{\mathrm{d}}}+\left(v T_{\mathrm{m}}+r_{\mathrm{d}}\right) .
$$

Then, the MUE HF probability is calculated differently for the following four cases. 
1) $v T_{\mathrm{m}}<R-r_{\mathrm{m}}-v T_{\mathrm{d}}$ : We know that for $\theta=0$, minimum value of $d_{\mathrm{HF}, \mathrm{m}}\left(\theta, R, r_{\mathrm{m}}\right)$ is obtained; i.e., $d_{\mathrm{HF}, \mathrm{m}}\left(0, R, r_{\mathrm{m}}\right)=d_{\mathrm{HF}, \min }=R-r_{\mathrm{m}}$. If the distance traveled by MUE $v T_{\mathrm{m}}+r_{\mathrm{d}}$ is less than $d_{\mathrm{HF}, \mathrm{min}}$, then there will be no MUE HF, i.e., $\mathcal{P}_{\mathrm{HF}, \mathrm{m}}=0$. This is because tracing the MUE trajectory with all possible angle values will not make MUE trajectory to overlap with MUE HF circle. In other words, for sufficiently low velocities, the SINR of the MUE will not be severely degraded before the TTT expires and handover is completed.

2) $R-r_{\mathrm{m}}<v T_{\mathrm{m}}+v T_{\mathrm{d}}<\sqrt{R^{2}-r_{\mathrm{m}}^{2}}$ : When UE velocity is high and the MUE HF condition in (3.17) is satisfied, then the MUE HF probability is expressed as

$$
\begin{aligned}
P_{\mathrm{HF}, \mathrm{m}} & =\mathbb{P}\left(l(\theta)>\frac{R^{2}-r_{\mathrm{m}}^{2}}{v T_{\mathrm{m}}+r_{\mathrm{d}}}+\left(v T_{\mathrm{m}}+r_{\mathrm{d}}\right)\right)=\int_{0}^{v T_{\mathrm{d}}} \frac{1}{v T_{\mathrm{d}}} \int_{\frac{R^{2}-r_{\mathrm{m}}^{2}}{v T_{\mathrm{m}}}+v T_{\mathrm{m}}^{2}+r_{\mathrm{d}}}^{\frac{R^{2}-r^{2}}{v T_{\mathrm{m}}+r_{\mathrm{d}}}} \frac{2}{\sqrt{4 R^{2}-T_{\mathrm{m}}^{2}}} \mathrm{~d} l \mathrm{~d}_{\mathrm{d}}+\int_{\frac{R^{2}-r_{\mathrm{m}}^{2}}{v T_{\mathrm{m}}}+v T_{\mathrm{m}}}^{2 R} \frac{2}{\pi \sqrt{4 R^{2}-l^{2}}} \mathrm{~d} l \mathrm{~d} r_{\mathrm{d}} \\
& =1-\int_{0}^{v T_{\mathrm{d}}} \frac{2}{\pi v T_{\mathrm{d}}} I_{1}\left(r_{\mathrm{d}}\right) \mathrm{d} r_{\mathrm{d}}
\end{aligned}
$$

where,

$$
I_{1}\left(r_{\mathrm{d}}\right)=\tan ^{-1}\left(\frac{R^{2}-r_{\mathrm{m}}^{2}+\left(v T_{\mathrm{m}}+r_{\mathrm{d}}\right)^{2}}{\sqrt{4 R^{2}\left(v T_{\mathrm{m}}+r_{\mathrm{d}}\right)^{2}-\left(R^{2}-r_{\mathrm{m}}^{2}+\left(v T_{\mathrm{m}}+r_{\mathrm{d}}\right)^{2}\right)^{2}}}\right) .
$$

3) $v T_{\mathrm{m}}>\sqrt{R^{2}-r_{\mathrm{m}}^{2}}-v T_{\mathrm{d}}$ : For this case, the MUE HF probability is the same as the MUE HF probability for case 2).

4) If the UE velocity is high, making the product $v T_{\mathrm{m}}$ greater than the chord length $2 \sqrt{R^{2}-r_{\mathrm{m}}^{2}}$, then the random variable $r_{\mathrm{d}}$ will have no effect and the MUE HF probability will be constant. Using (3.5) we can write MUE HF probability as

$$
\mathcal{P}_{\mathrm{NHO}}=\mathbb{P}\left(l(\theta)>2 \sqrt{R^{2}-r_{\mathrm{m}}^{2}}\right)=\int_{2 \sqrt{R^{2}-r_{\mathrm{m}}^{2}}}^{2 R} \frac{2}{\pi \sqrt{4 R^{2}-l^{2}}} \mathrm{~d} l=1-\frac{2}{\pi} \tan ^{-1}\left(\frac{\sqrt{R^{2}-r_{\mathrm{m}}^{2}}}{r_{\mathrm{m}}}\right) .
$$




\subsubsection{HF Probability for PUEs}

In order to observe a PUE HF, there should be a successful handover of MUE to the picocell first. After a successful handover to the picocell, the PUE continues gathering measurements from the neighbouring cells. If a PUE enters the coverage of the macrocell and the handover event entry condition is satsified (e.g., L3 filtered RSRP of the macro cell is larger than that of the picocell plus a hysteresis parameter), then TTT of duration $T_{\mathrm{p}}$ is triggered (see Fig. 3.7). For simplicity, we assume that the discrete offset random variable $r_{\mathrm{d}}$ is the same whenever there is a handover to picocell or macrocell.

If a PUE reaches the PUE HF circle before the TTT expires, there will be a PUE HF. In other words, a PUE HF occurs when the total distance travelled by PUE $\left(v T_{\mathrm{p}}+r_{\mathrm{d}}\right)$ is greater than the distance $d_{\mathrm{HF}, \mathrm{p}}\left(\theta, R, r_{\mathrm{p}}\right)$. If we consider a point on the ideal picocell coverage area where the UE starts entering the coverage of the macrocell after a successful handover to picocell, then distance from this point to the PUE HF circle is given by

$$
d_{\mathrm{HF}, \mathrm{p}}\left(\theta, R, r_{\mathrm{p}}\right)=R \cos (\theta)+\sqrt{r_{\mathrm{p}}^{2}-R^{2} \sin ^{2}(\theta)}-l(\theta) .
$$

To obtain the PUE HF probability, we evaluate the PUE HF condition

$$
v T_{\mathrm{p}}+r_{\mathrm{d}}>d_{\mathrm{HF}, \mathrm{p}}\left(\theta, R, r_{\mathrm{p}}\right)
$$

in terms of UE trajectory $l(\theta)$ like we did before for MUE HF. Using (3.21) with (3.22), we get the condition for observing PUE HF as

$$
l(\theta)>\frac{r_{\mathrm{p}}^{2}-R^{2}}{v T_{\mathrm{p}}+r_{\mathrm{d}}}-\left(v T_{\mathrm{p}}+r_{\mathrm{d}}\right) .
$$

Based on the condition in (3.23) and the condition $l(\theta)>2 \sqrt{R^{2}-r_{\mathrm{m}}^{2}}$, we can show that there will be a PUE HF when $v T_{\mathrm{m}}+v T_{\mathrm{p}}>\sqrt{r_{\mathrm{p}}^{2}-r_{\mathrm{m}}^{2}}-\sqrt{R^{2}-r_{\mathrm{m}}^{2}}$. For different values of $v T_{\mathrm{m}}$ and $v T_{\mathrm{d}}$, the PUE HF is given as follows. 
1) $v T_{\mathrm{m}}+v T_{\mathrm{d}}<\sqrt{R^{2}-r_{\mathrm{m}}^{2}}$ : In this case, in order to observe PUE HF, first of all there needs to be no MUE HF. Then, we can use the MUE HF condition in (3.17) and the PUE HF condition in (3.23) to obtain PUE HF. There will be a possible PUE HF for the condition

$$
v T_{\mathrm{m}}+v T_{\mathrm{p}}>\sqrt{r_{\mathrm{p}}^{2}-r_{\mathrm{m}}^{2}}-\sqrt{R^{2}-r_{\mathrm{m}}^{2}} .
$$

After some manipulation, the PUE HF probability is given by

$$
\begin{aligned}
P_{\mathrm{HF}, \mathrm{p}} & =\mathbb{P}\left(\max \left(v T_{\mathrm{m}}+r_{\mathrm{d}}, \frac{r_{\mathrm{p}}^{2}-R^{2}}{v T_{\mathrm{p}}+r_{\mathrm{d}}}-\left(v T_{\mathrm{p}}+r_{\mathrm{d}}\right)\right)\right. \\
& \left.<l(\theta)<\min \left(\frac{R^{2}-r_{\mathrm{m}}^{2}}{v T_{\mathrm{m}}+r_{\mathrm{d}}}+\left(v T_{\mathrm{m}}+r_{\mathrm{d}}\right), 2 R\right)\right) .
\end{aligned}
$$

In other words, (3.25) implies that for a successful handover to happen for the MUE, and for a HF to happen for the PUE, the chord of the picocell coverage circle, with length $l(\theta)$, should be in between the two values as given in (3.25).

Let us now consider the following definitions for brevity:

$$
\begin{aligned}
& d_{\mathrm{rp}}=\frac{r_{\mathrm{p}}^{2}-R^{2}}{v T_{\mathrm{p}}+r_{\mathrm{d}}}-\left(v T_{\mathrm{p}}+r_{\mathrm{d}}\right), \\
& d_{\mathrm{rm}}=\frac{R^{2}-r_{\mathrm{m}}^{2}}{v T_{\mathrm{m}}+r_{\mathrm{d}}}+\left(v T_{\mathrm{m}}+r_{\mathrm{d}}\right), \\
& d_{\mathrm{vm}}=v T_{\mathrm{m}}+r_{\mathrm{d}} .
\end{aligned}
$$

Using (3.26)-(3.28), we obtain that $L_{\mathrm{p}}=\max \left(d_{\mathrm{vm}}, d_{\mathrm{rp}}\right)$ and $L_{\mathrm{m}}=\min \left(d_{\mathrm{rm}}, 2 R\right)$. Using the PDF in (3.5), we can calculate the PUE HF probability after some manipulation as 


$$
\begin{aligned}
& P_{\mathrm{HF}, \mathrm{p}}=\int_{0}^{v T_{\mathrm{d}}} \frac{1}{v T_{\mathrm{d}}} \int_{L_{\mathrm{p}}}^{L_{\mathrm{m}}} \frac{2}{\pi \sqrt{4 R^{2}-l^{2}}} \mathrm{~d} l \mathrm{~d} r_{\mathrm{d}} \\
& =\int_{0}^{R-r_{\mathrm{m}}-v T_{\mathrm{m}}} \frac{1}{v T_{\mathrm{d}}} \mathrm{d} r_{\mathrm{d}}+\int_{R-r_{\mathrm{m}}-v T_{\mathrm{m}}}^{v T_{\mathrm{d}}} \frac{2}{\pi v T_{\mathrm{d}}} \tan ^{-1}\left(\frac{d_{\mathrm{rm}}}{\sqrt{4 R^{2}-d_{\mathrm{rm}}^{2}}}\right) \mathrm{d} r \int_{0}^{l_{\mathrm{p}}} \tan ^{-1}\left(\frac{d_{\mathrm{rp}}}{\sqrt{4 R^{2}-d_{\mathrm{rp}}^{2}}}\right) \mathrm{d} r_{\mathrm{d}} \\
& -\int_{l_{\mathrm{p}}}^{v T_{\mathrm{d}}} \tan ^{-1}\left(\frac{d_{\mathrm{vm}}}{\sqrt{4 R^{2}-d_{\mathrm{vm}}^{2}}}\right) \mathrm{d} r_{\mathrm{d}}
\end{aligned}
$$

where,

$$
l_{\mathrm{p}}=\frac{\sqrt{-8 R^{2}+8 r_{\mathrm{p}}^{2}+\left(v T_{\mathrm{m}}\right)^{2}-2 v^{2} T_{\mathrm{m}} T_{\mathrm{p}}+\left(v T_{\mathrm{p}}\right)^{2}-3 v^{2} T_{\mathrm{m}} T_{\mathrm{p}}}}{4} .
$$

In $(3.30), l_{\mathrm{p}}$ is the value of $r_{\mathrm{d}}$ which results in $d_{\mathrm{vm}}=d_{\mathrm{rp}}$.

2) $\sqrt{R^{2}-r_{\mathrm{m}}^{2}}<v T_{\mathrm{m}}+v T_{\mathrm{d}}<2 \sqrt{R^{2}-r_{\mathrm{m}}^{2}}$ : In this case, MUE HF occurs when $l(\theta)>2 \sqrt{R^{2}-r_{\mathrm{m}}^{2}}$. Using this condition and the PUE HF condition in (3.23), the PUE HF probability is given by

$$
\begin{array}{r}
P_{\mathrm{HF}, \mathrm{p}}=\mathbb{P}\left(\max \left(v T_{\mathrm{m}}+r_{\mathrm{d}}, \frac{r_{\mathrm{p}}^{2}-R^{2}}{v T_{\mathrm{p}}+r_{\mathrm{d}}}-\left(v T_{\mathrm{p}}+r_{\mathrm{d}}\right)\right)\right. \\
\left.<l(\theta)<2 \sqrt{R^{2}-r_{\mathrm{m}}^{2}}\right) .
\end{array}
$$

Using (3.5), (3.26)-(3.28), $L_{\mathrm{m}}$ and $L_{\mathrm{p}}$, we can find PUE HF probability after some manipulation as

$$
\begin{aligned}
P_{\mathrm{HF}, \mathrm{p}} & =\int_{0}^{v T_{\mathrm{d}}} \frac{1}{v T_{\mathrm{d}}} \int_{L_{\mathrm{p}}}^{r_{\mathrm{m}}} \frac{2}{\pi \sqrt{4 R^{2}-l^{2}}} \mathrm{~d} l \mathrm{~d} r_{\mathrm{d}} \\
& =\frac{2}{\pi} \tan ^{-1}\left(\frac{\sqrt{R^{2}-r_{\mathrm{m}}^{2}}}{r_{\mathrm{m}}}\right)+\int_{0}^{l_{\mathrm{p}}} \tan ^{-1}\left(\frac{d_{\mathrm{rp}}}{\sqrt{4 R^{2}-d_{\mathrm{rp}}^{2}}}\right) \mathrm{d} r_{\mathrm{d}}+\int_{l_{\mathrm{p}}}^{v T_{\mathrm{d}}} \tan ^{-1}\left(\frac{d_{\mathrm{vm}}}{\sqrt{4 R^{2}-d_{\mathrm{vm}}^{2}}}\right) \mathrm{d} r_{\mathrm{d}} .
\end{aligned}
$$


3) $v T_{\mathrm{m}}+v T_{\mathrm{d}}>2 \sqrt{R^{2}-r_{\mathrm{m}}^{2}}$ : In this case, PUE HF probability is the same as in case 2 of PUE HF probability.

\subsection{Handover Failure Analysis with Fading}

The channel impairments might degrade the handover performance of the UEs in cellular networks. In particular, channel fading causes the link qualities from the neighboring cells to fluctuate in a random manner; as a result and depending on the L1/L3 sampling period, it varies when and where the handover trigger events occur. In this research work, we model the HF locations in the fading scenario as a circle, since the wideband SINR (which dictates handover failures) is typically averaged over a large bandwidth, and the effect of fading is mitigated. On the contrary, the handover trigger locations in the fading scenario depend on the RSRP, which may be measured over a narrow bandwidth of six resource blocks in LTE and thus not as reliable. Therefore, RSRP is subject to larger randomness, which may significantly affect the handover trigger locations.

\subsubsection{Modeling the Handover Trigger Locations in the Fad- ing Scenario}

In this research work, we use a $3 \mathrm{GPP}$ compliant system level simulator with channel fading to extract statistics related to handover trigger locations, for analyzing handover performance in fading environments. A typical urban (TU) channel model based on the modeling assumptions in [86] was used to implement the UE channel in outdoor hotspot scenarios. The TU channel is designed to model the time variations of the channel, which arise due to the UEs' motion and other impairments. The channel fluctuations will increase with the UE's velocity, which will impact handover 
performance. Based on this scenario, a representative set of handover trigger locations of a UE around the coverage area of a picocell are illustrated in Fig. 3.11 using system level simulations.

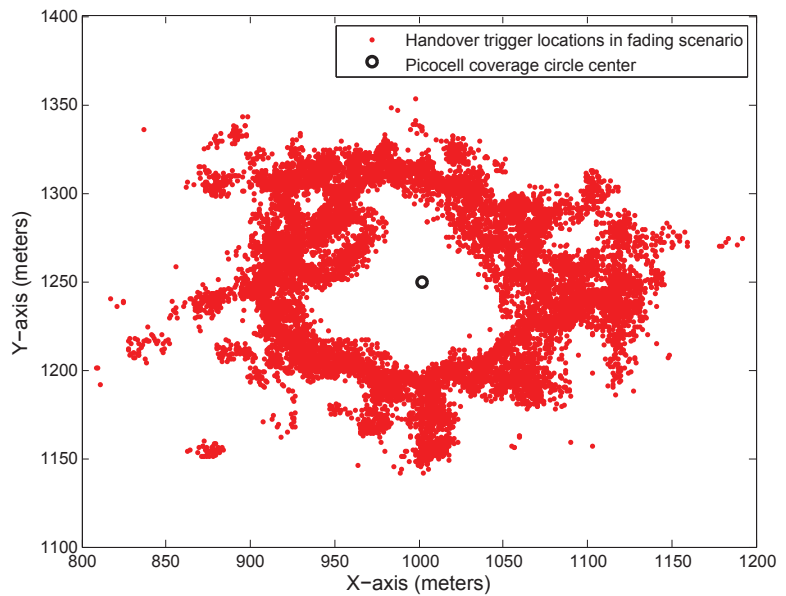

Figure 3.11: Handover locations in the fast-fading and shadowing scenario (Case 3) for UE velocity $v=60 \mathrm{~km} / \mathrm{h}$ and $T_{\mathrm{d}}=40 \mathrm{~ms}$.

Comparing Fig. 3.11 with Fig. 3.6, we can see that due to fading channel conditions, handover trigger locations in Fig. 3.11 are not delimited within the coverage boundaries of the picocell. In other words, TTT timer can be initiated for locations that are far away from the ideal picocell coverage area. To model the statistics of the handover trigger locations with L1/L3 filtering, we examine the distance of each handover trigger location shown in Fig. 3.11 from the ideal picocell coverage boundary, which we define as handover offset distance. We model this distance using a random variable denoted by $\hat{r}_{\mathrm{d}}$ and obtain histograms for it as shown in Fig. 3.12. The negative distance in histograms is due to the possibility of handover trigger locations being outside the ideal picocell coverage area due to fading.

After testing several standard distributions, we conclude that there is no standard distribution that reasonably approximates the histograms in Fig. 3.12. Therefore, for the fading scenario, we directly use the histogram data to obtain the semi-analytic 


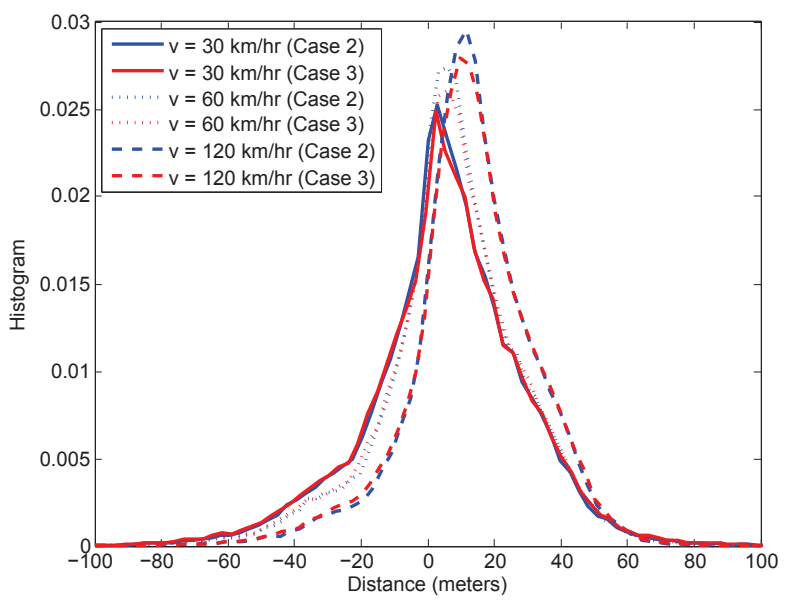

Figure 3.12: Handover offset distance histograms for $T_{\mathrm{d}}=40 \mathrm{~ms}$ in fading scenario.

HF expressions for MUEs and PUEs. The histograms for $\hat{r}_{\mathrm{d}}$ are given by $f\left(\hat{r}_{\mathrm{d}}\right)$, where $\hat{r}_{\mathrm{d}} \in\left(\hat{r}_{\min }, \hat{r}_{\max }\right)$.

\subsubsection{HF Probability Analysis for MUEs}

The derivation of MUE HF probabilities in shadowing and fast-fading is carried in a similar manner to that of the ideal handover model case. The only difference comes with the distribution of the random variable $\hat{r}_{\mathrm{d}}$, which is obtained directly from the histograms as discussed in Section 3.4.1. The MUE HF probability for a given UE can be calculated as

$$
\begin{aligned}
& \mathbb{P}\left(l(\theta)>\frac{R^{2}-r_{\mathrm{m}}^{2}}{v T_{\mathrm{m}}+\hat{r}_{\mathrm{d}}}+\left(v T_{\mathrm{m}}+\hat{r}_{\mathrm{d}}\right)\right) \\
& =\int_{\hat{r}_{\min }}^{\hat{r}_{\max }} f\left(\hat{r}_{\mathrm{d}}\right) \mathrm{d} \hat{r}_{\mathrm{d}} \int_{\hat{d}_{\mathrm{rm}}}^{2 R} \frac{2}{\pi \sqrt{4 R^{2}-l^{2}}} \mathrm{~d} l=\int_{\hat{r}_{\min }}^{\hat{r}_{\max }} f\left(\hat{r}_{\mathrm{d}}\right) d \hat{r}_{\mathrm{d}}-\int_{\hat{r}_{\min }}^{\hat{r}_{\max }} I_{1}\left(\hat{r}_{\mathrm{d}}\right) d \hat{r}_{\mathrm{d}} \\
& =\int_{\hat{r}_{\min }}^{\hat{r}_{\max }} f\left(\hat{r}_{\mathrm{d}}\right) d \hat{r}_{\mathrm{d}}-\int_{R-r_{\mathrm{m}}-v T_{\mathrm{m}}}^{\sqrt{R^{2}-r_{\mathrm{m}}^{2}}-v T_{\mathrm{m}}} I_{1}\left(\hat{r}_{\mathrm{d}}\right) f\left(\hat{r}_{\mathrm{d}}\right) d \hat{r}_{\mathrm{d}}-\int_{\sqrt{R^{2}-r_{\mathrm{m}}^{2}}-v T_{\mathrm{m}}}^{\hat{r}_{\max }} \tan ^{-1}\left(\frac{\sqrt{R^{2}-r_{\mathrm{m}}^{2}}}{r_{\mathrm{m}}}\right) f\left(\hat{r}_{\mathrm{d}}\right) d \hat{r}_{\mathrm{d}}-\int_{\hat{r}_{\min }}^{R-r_{\mathrm{m}}-v T_{\mathrm{m}}} f\left(\hat{r}_{\mathrm{d}}\right) d \hat{r}_{\mathrm{d}},
\end{aligned}
$$


where,

$$
I_{1}\left(\hat{r}_{\mathrm{d}}\right)=\tan ^{-1}\left(\frac{R^{2}-r_{\mathrm{m}}^{2}+\left(v T_{\mathrm{m}}+\hat{r}_{\mathrm{d}}\right)^{2}}{\sqrt{4 R^{2}\left(v T_{\mathrm{m}}+\hat{r}_{\mathrm{d}}\right)^{2}-\left(R^{2}-r_{\mathrm{m}}^{2}+\left(v T_{\mathrm{m}}+\hat{r}_{\mathrm{d}}\right)^{2}\right)^{2}}}\right) .
$$

In Section 3.5, the above integrals are solved numerically to provide numerical results for $\mathrm{HF}$ probabilities in the presence of fading.

\subsubsection{HF Probability Analysis for PUEs}

The derivation of PUE HF probabilities in shadowing and fast-fading is carried in a similar manner to that of the ideal handover model case. The only difference comes with the distribution of the random variable $\hat{r}_{\mathrm{d}}$, which is obtained directly from the histograms as discussed in Section 3.4.1. Using the MUE HF condition in (3.17), PUE HF condition in (3.23), and after some manipulation, the PUE HF probability for a given UE can be calculated as

$$
\begin{aligned}
P_{\mathrm{HF}, \mathrm{p}} & =\mathbb{P}\left(\max \left(v T_{\mathrm{m}}+\hat{r}_{\mathrm{d}}, \frac{r_{\mathrm{p}}^{2}-R^{2}}{v T_{\mathrm{p}}+r_{\mathrm{d}}}-\left(v T_{\mathrm{p}}+\hat{r}_{\mathrm{d}}\right)\right)\right. \\
& \left.<l(\theta)<\min \left(\frac{R^{2}-r_{\mathrm{m}}^{2}}{v T_{\mathrm{m}}+\hat{r}_{\mathrm{d}}}+\left(v T_{\mathrm{m}}+\hat{r}_{\mathrm{d}}\right), 2 R\right)\right) .
\end{aligned}
$$

Considering the following definitions for brevity:

$$
\begin{aligned}
& \hat{d}_{\mathrm{rp}}=\frac{r_{\mathrm{p}}^{2}-R^{2}}{v T_{\mathrm{p}}+\hat{r}_{\mathrm{d}}}-\left(v T_{\mathrm{p}}+\hat{r}_{\mathrm{d}}\right), \\
& \hat{d}_{\mathrm{rm}}=\frac{R^{2}-r_{\mathrm{m}}^{2}}{v T_{\mathrm{m}}+\hat{r}_{\mathrm{d}}}+\left(v T_{\mathrm{m}}+\hat{r}_{\mathrm{d}}\right), \\
& \hat{d}_{\mathrm{vm}}=v T_{\mathrm{m}}+\hat{r}_{\mathrm{d}},
\end{aligned}
$$

we can write that $\hat{L}_{\mathrm{p}}=\max \left(d_{\mathrm{vm}}, d_{\mathrm{rp}}\right)$ and $\hat{L}_{\mathrm{m}}=\min \left(d_{\mathrm{rm}}, 2 R\right)$. Using the PDF in (3.5), we can calculate the PUE HF probability as 


$$
\begin{aligned}
& P_{\mathrm{HF}, \mathrm{p}}=\int_{\hat{r}_{\min }}^{\hat{r}_{\max }} f\left(\hat{r}_{\mathrm{d}}\right) \int_{L_{\mathrm{p}}}^{L_{\mathrm{m}}} \frac{2}{\pi \sqrt{4 R^{2}-l^{2}}} \mathrm{~d} l \mathrm{~d} \hat{r}_{\mathrm{d}} \\
& =\int_{\hat{r}_{\min }}^{R-r_{\mathrm{m}}-v T_{\mathrm{m}}} \frac{1}{v T_{\mathrm{d}}} \mathrm{d} \hat{r}_{\mathrm{d}}+\int_{R-r_{\mathrm{m}}-v T_{\mathrm{m}}}^{\sqrt{R^{2}-r_{\mathrm{m}}^{2}}-v T_{\mathrm{m}}} \frac{2}{\pi v T_{\mathrm{d}}} \tan ^{-1}\left(\frac{\hat{d}_{\mathrm{rm}}}{\sqrt{4 R^{2}-\hat{d}_{\mathrm{rm}}^{2}}}\right) \mathrm{d} \hat{r}_{\mathrm{d}}+\int_{\sqrt{R^{2}-r_{\mathrm{m}}^{2}}-v T_{\mathrm{m}}}^{\hat{r}_{\max }} \tan ^{-1}\left(\frac{\sqrt{R^{2}-r_{\mathrm{m}}^{2}}}{r_{\mathrm{m}}}\right) f\left(\hat{r}_{\mathrm{d}}\right) \mathrm{d} \hat{r}_{\mathrm{d}} \\
& -\int_{\hat{r}_{\min }}^{r_{\mathrm{p}}-R-v T_{\mathrm{p}}} f\left(\hat{r}_{\mathrm{d}}\right) \mathrm{d} \hat{r}_{\mathrm{d}}-\int_{2 R-v T_{\mathrm{m}}}^{\hat{r}_{\max }} f\left(\hat{r}_{\mathrm{d}}\right) \mathrm{d} \hat{r}_{\mathrm{d}}-\int_{r_{\mathrm{p}}-R-v T_{\mathrm{p}}}^{l_{\mathrm{p}}} \tan ^{-1}\left(\frac{\hat{d}_{\mathrm{rp}}}{\sqrt{4 R^{2}-\hat{d}_{\mathrm{rp}}^{2}}}\right) \mathrm{d} \hat{r}_{\mathrm{d}} \\
& -\int_{l_{\mathrm{p}}}^{2 R-v T_{\mathrm{m}}} \tan ^{-1}\left(\frac{d_{\mathrm{vm}}}{\sqrt{4 R^{2}-d_{\mathrm{vm}}^{2}}}\right) \mathrm{d} \hat{r}_{\mathrm{d}}
\end{aligned}
$$

Again, to obtain numerical results in Section 3.5, the above integrals are solved numerically using the histograms $f\left(r_{d}\right)$ from Fig. 3.12.

\subsection{Numerical Results}

The results are based on the analytical derivations described in earlier sections. In order to validate our analysis, computer simulations are carried out. Initially, as shown in Fig. 3.7, we fix the starting position of the MUE to a reference point on the picocell coverage circle. The MUE travels a distance equal to $v T_{\mathrm{m}}+r_{\mathrm{d}}$ from the reference point with different realizations of angle $\theta$, which is uniformly distributed in $\left[-\frac{\pi}{2}, \frac{\pi}{2}\right]$. Since $r_{\mathrm{d}}$ is uniformly distributed between $\left[0, v T_{\mathrm{d}}\right]$, we have different values

of $r_{\mathrm{d}}$ for each $\theta$. After the MUE has traveled a distance equal to $v T_{\mathrm{m}}+r_{\mathrm{d}}$, the final point of the MUE is checked for intersection with MUE HF circle. If it intersects with the MUE HF circle then there is a MUE HF. We aggregate all these MUE HF events and normalize them over $\theta$ and $r_{\mathrm{d}}$ relizations to obtain MUE HF probabilities for each UE velocity.

In order to obtain PUE HF probability, we find the end points of the chord using $l(\theta)=2 R \cos (\theta)$ for the picocell coverage circle for different $\theta$ realizations. Then, we 
take those points as the reference point, and fix the starting position for the PUE. Subsequently, the PUE travels a distance equal to $v T_{\mathrm{p}}+r_{\mathrm{d}}$ and the final point of the PUE is checked for intersection with the PUE HF circle. If it intersects with the PUE HF circle, then there is a PUE HF. We aggregate all these PUE HF events and normalize them over $\theta$ and $r_{\mathrm{d}}$ realizations to obtain PUE HF probabilities for each UE velocity.

In the following subsection the MUE and PUE HF probabilities are shown for $R=64 \mathrm{~m}, r_{\mathrm{m}}=50 \mathrm{~m}$ and $r_{\mathrm{p}}=78 \mathrm{~m}$ in the no fading and fading scenarios.

\subsubsection{Results with No Fading}

Using the above simulation assumptions, theoretical MUE HF and PUE HF probabilities derived in Section 3.3 are plotted as a function of UE velocity and are verified via simulation results. Initially, it is assumed there is no fading or shadowing.

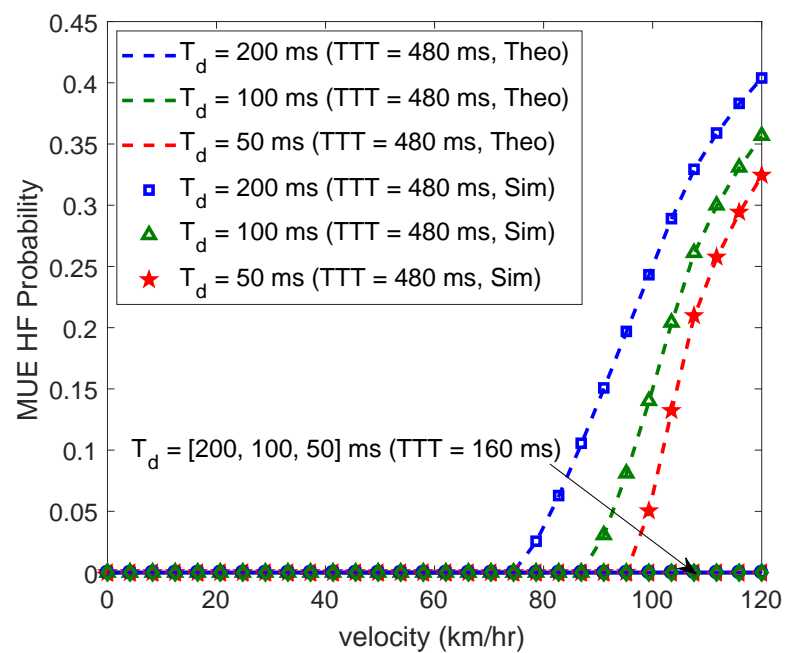

(a) MUE HF probability for non-overlapping measurement filtering approach.

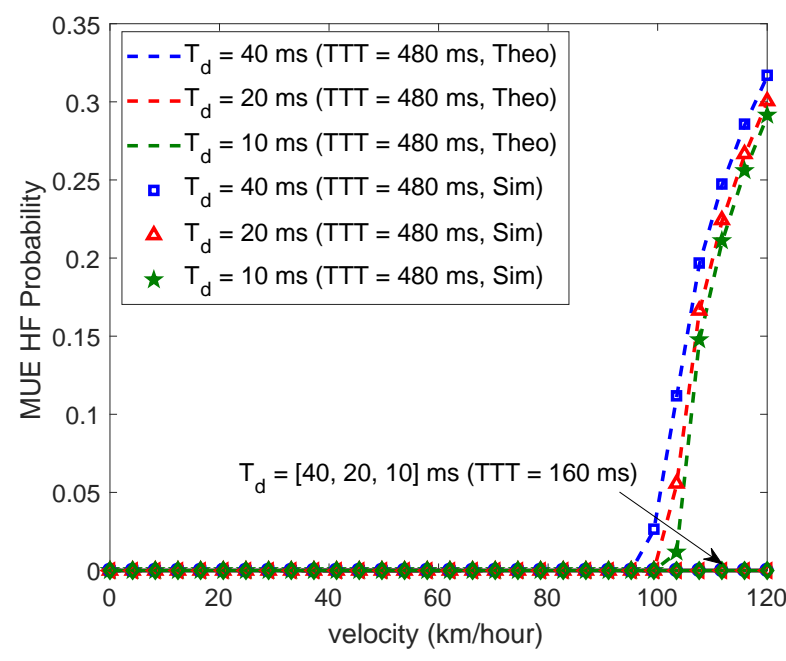

(b) MUE HF probability for sliding window filtering approach.

Figure 3.13: Theoretical (lines) and simulated (markers) no fading results as a function of UE velocity for $R=64 \mathrm{~m}, r_{\mathrm{m}}=50 \mathrm{~m}$ and $r_{\mathrm{p}}=78 \mathrm{~m}$ in the case of non-overlapping handover filtering process [7]. 
The MUE HF probability for the non-overlapping handover filtering process in [7] and the sliding window filtering stated in 3GPP are shown in Fig. 3.13(a) and Fig. 3.13(b), respectively.

The MUE HF probability for the non-overlapping filtering approach is shown for different TTT and $T_{\mathrm{d}}=[200,100,50] \mathrm{ms}$. We see that as UE velocity increases, the MUE HF probability increases. On the other hand, when the sampling period of L3 filter decreases, the MUE HF probability decreases, since the TTT can be initiated earlier. For example, the MUE HF probability for UE velocity $80 \mathrm{~km} / \mathrm{hr}$ improves by approximately 10 percent when sampling period is reduced from $150 \mathrm{~ms}$ to $50 \mathrm{~ms}$. By reducing TTT to $160 \mathrm{~ms}$ we can see that the MUE HF probability becomes almost negligible. This is because the UEs finalize the handover in a quicker way, when compared to larger TTT values.

The MUE HF probability for the sliding window handover filtering shown in Fig. 3.13(b) is lower compared to the non-overlapping filtering process. This is due to the lower values of $T_{\mathrm{d}}=[40,20,10] \mathrm{ms}$, which implies frequent measurements performed at the UE. We see that the improvement in the MUE HF probability for the UE velocity $120 \mathrm{~km} / \mathrm{hr}$ is 2.5 percent when the sampling period $T_{\mathrm{d}}$ is reduced from $40 \mathrm{~ms}$ to $10 \mathrm{~ms}$. On the other hand, when $T_{\mathrm{d}}$ is reduced from $200 \mathrm{~ms}$ to $50 \mathrm{~ms}$, MUE $\mathrm{HF}$ is improved by 7.5 percent for the non-overlapping filtering approach.

PUE HF for different TTT and $T_{\mathrm{d}}$ values in the case of non-overlapping handover filtering process is shown in Fig. 3.14(a). We can see that as UE velocity increases, PUE HF probability increases and it is improved when sampling period $T_{\mathrm{d}}$ decreases. For example, the PUE HF probability for UE velocity $120 \mathrm{~km} / \mathrm{hr}$ improves by approximately 5 percent when sampling period is reduced from $150 \mathrm{~ms}$ to $50 \mathrm{~ms}$. By reducing the TTT to $160 \mathrm{~ms}$ we can see that there will be no PUE HF probability when no fast fading and shadowing are considered in analysis/simulations. 


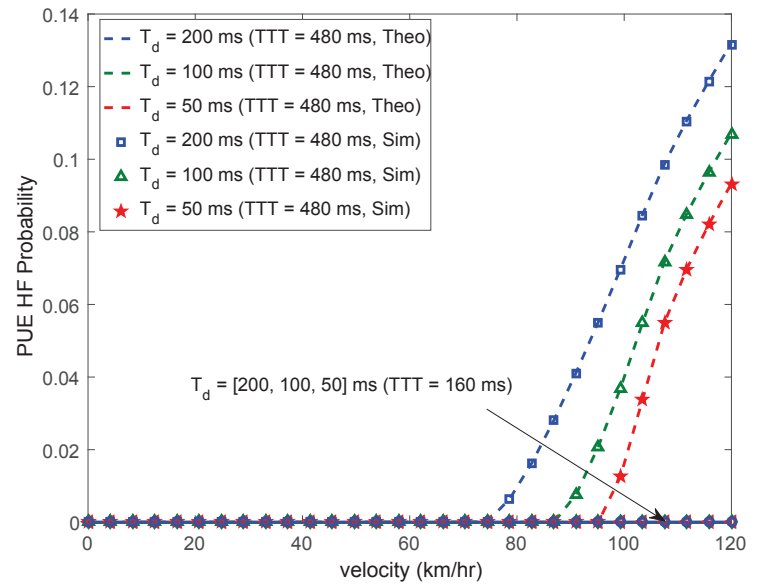

(a) PUE HF probability for non-overlapping measurement $($ b) filtering approach.

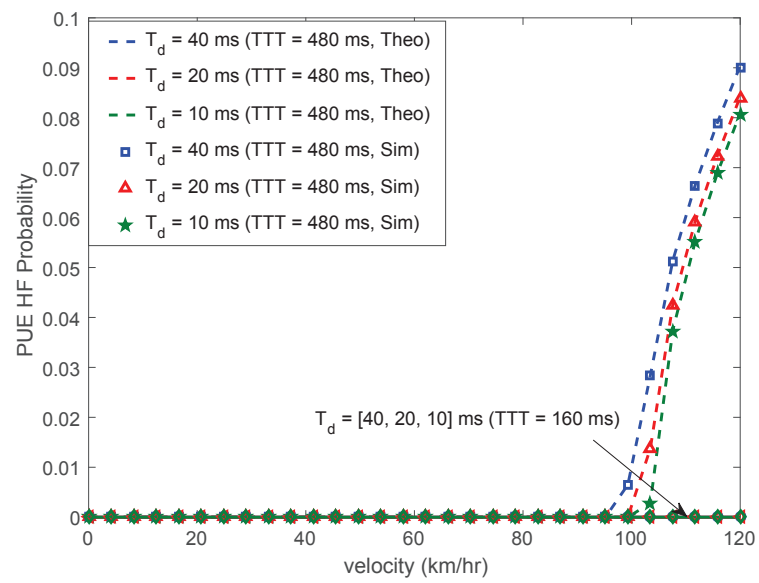

(b) PUE HF probability for sliding window filtering approach.

Figure 3.14: Theoretical (lines) and simulated (markers) no fading results as a function of UE velocity for $R=64 \mathrm{~m}, r_{\mathrm{m}}=50 \mathrm{~m}$ and $r_{\mathrm{p}}=78 \mathrm{~m}$.

The PUE HF probability for different TTT values and for $T_{\mathrm{d}}=[40,20,10] \mathrm{ms}$ in the sliding window filtering approach is shown in Fig. 3.14(b). The PUE HF probability is improved due to the lower values of sampling period $T_{\mathrm{d}}$. Moreover, there is less improvement in the PUE HF probability when $T_{\mathrm{d}}$ is decreased compared to the non-overlapping filtering approach.

The downside of reducing the sampling period of L1/L3 filter is that it increases the unnecessary handovers called ping-pongs shown in Fig. 3.15(a) for the nonoverlapping filtering process. We can see that ping-pong probability increases when the sampling period of L1/L3 filter is reduced from $200 \mathrm{~ms}$ to $50 \mathrm{~ms}$. The reason for this is that for a larger sampling period of L1/L3 filter, the output of the filter is more stable and the UE tends to stay with its serving cell for a longer time before initiating the handover process, which will naturally reduce ping-pong handovers.

The ping-pong probability for the sliding window filtering approach is shown in Fig. 3.15(b). The results show that there is a slight increase in ping-pong probability due to the frequent measurement performed at the UE. 

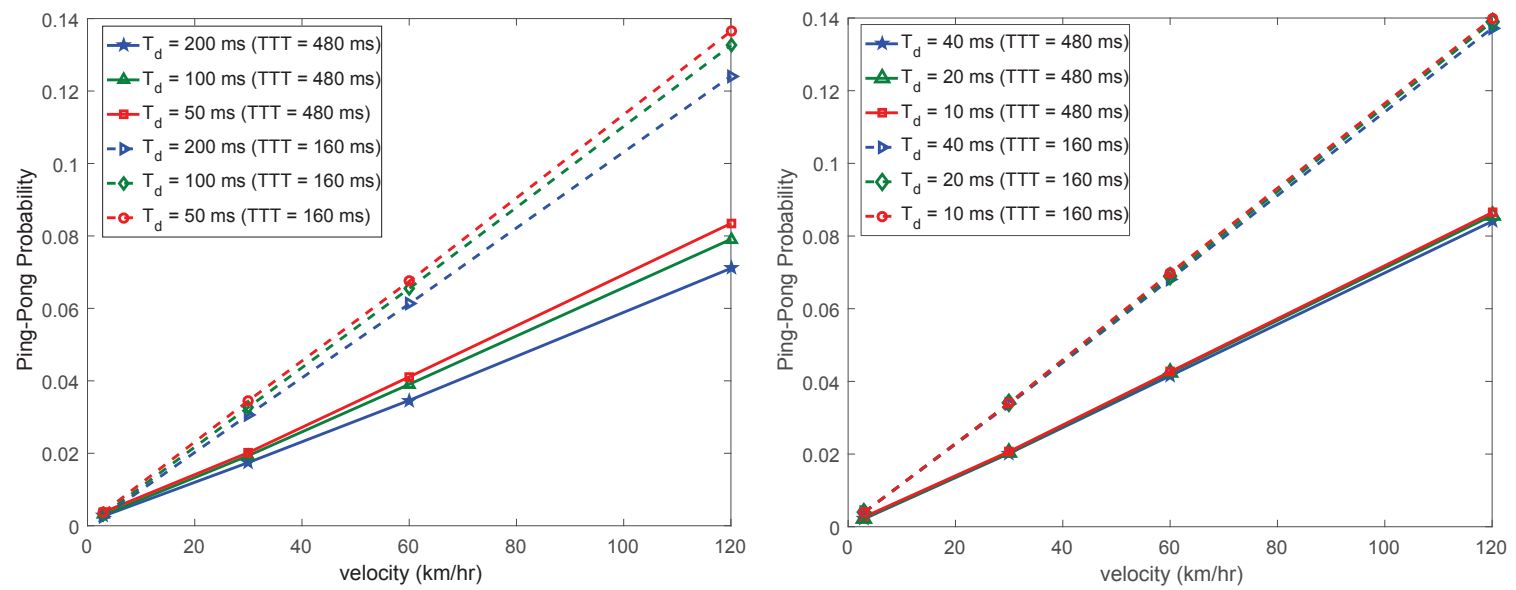

(a) Ping-pong simulation probabilities for non-overlapping(b) Ping-pong simulation probabilities for sliding window measurement filtering approach. filtering approach.

Figure 3.15: Ping-pong results as a function of UE velocity for $R=64 \mathrm{~m}, r_{\mathrm{m}}=50 \mathrm{~m}$ and $r_{\mathrm{p}}=78 \mathrm{~m}$.

\subsubsection{Results with Fading}

In order to obtain results with channel fading, a TU channel model is used as discussed in Section 3.4.1. In this case, we use the histogram data shown in Fig. 3.12 to generate samples for $\hat{r}_{\mathrm{d}}$, follow the other simulation assumptions stated previously, and plot the MUE HF and PUE HF probabilities as a function of UE velocity.

The MUE HF probability plots are shown in Fig. 3.16(a). We can see that MUE handover performance is degraded for all UE velocities compared to no-fading scenario in Fig. 3.13. Moreover, MUE handover performance is improved when the sampling period $\left(T_{\mathrm{d}}\right)$ of the filter is decreased. We can see that for MUE traveling with a velocity $120 \mathrm{~km} / \mathrm{h}$, the MUE HF is improved by 12.19 percent when sampling period of the filter is reduced from $200 \mathrm{~ms}$ to $50 \mathrm{~ms}$. Results show that even at low UE velocities, there may be on the order of $10 \% \mathrm{HF}$ probability. Note that these results consider a worst-case simulation scenario, in which the UE starts its path at the coverage area of a picocell base station as shown in Fig. 3.7. 

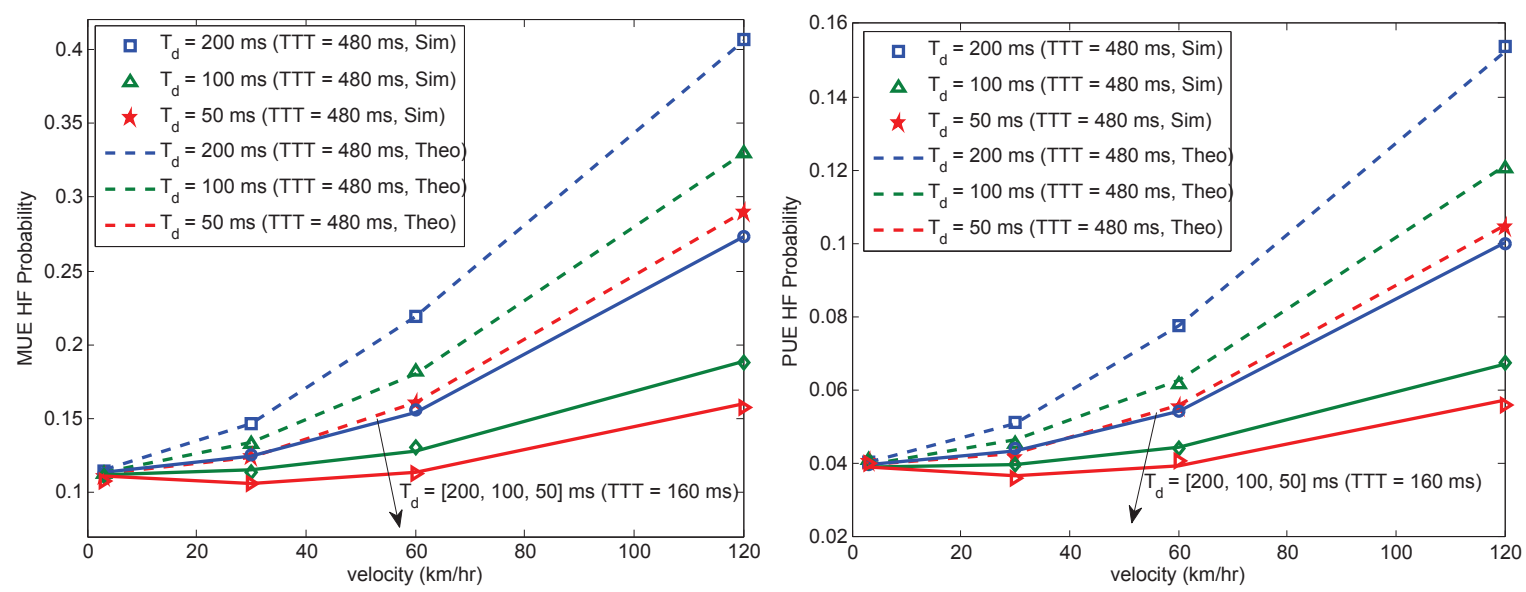

(a) MUE HF probability with fast-fading and shadowing(b) PUE HF probability with fast-fading and shadowing (Case 3).

(Case 3).

Figure 3.16: Theoretical (lines) and simulated (markers) no fading results as a function of UE velocity for $R=64 \mathrm{~m}, r_{\mathrm{m}}=50 \mathrm{~m}$ and $r_{\mathrm{p}}=78 \mathrm{~m}$.

The PUE HF probability plots are shown in Fig. 3.16(b). We notice that the PUE HF probability increases as UE velocity increases and it is improved when the sampling period $T_{\mathrm{d}}$ of the filter is decreased. The PUE HF probability improves by 3 percent for PUE traveling with a velocity $120 \mathrm{~km} / \mathrm{h}$ in the fading channel conditions. This is due to higher HF probabilities of MUEs at higher speeds.

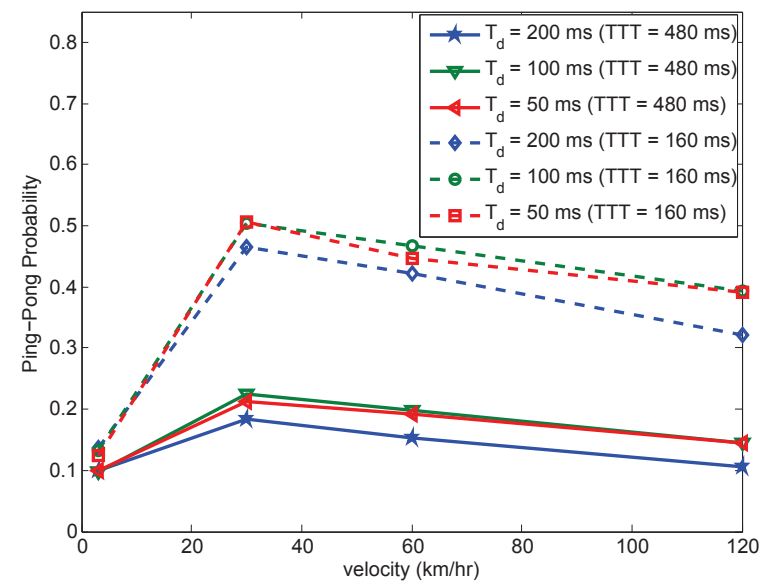

Figure 3.17: Simulation plots for fading ping-pong probabilities as a function of UE velocity for $R=64 \mathrm{~m}, r_{\mathrm{m}}=50 \mathrm{~m}$ and $r_{\mathrm{p}}=78 \mathrm{~m}$. 
In order to investigate the impact of TTT and $T_{\mathrm{d}}$ on ping-pong handover performance, ping-pong probability plots in the fading scenario are shown in Fig. 3.17. We can see that there are more ping-pongs when $T_{\mathrm{d}}$ and TTT are reduced, and this is because using shorter TTT and $T_{\mathrm{d}}$ the output of the filter is less reliable, creating oscillations in the server selection process. In the Fig. 3.17, we can see that there are more ping-pongs in fading scenario compared to no-fading scenario shown in Fig. 3.15a. This is because, in the case of fading scenario and assuming from the picocell perspective, the the link quality of the serving cell is more prone to fluctuations. As a result there might be handovers back and forth and UEs time-of-stay will be less than the ping-pong threshold causing more ping-pongs. 


\section{CHAPTER 4}

\section{FUZZY BASED GAME THEORETIC MOBILITY MANAGEMENT FOR ENERGY EFFICIENT OPERATION IN HETNETS}

In order to study the performance of the fuzzy based game theoretic mobility management scheme, we consider two-tier HetNet system model which consists of macro BS (MBS) and several overlaid small cell BSs (SBSs) as shown in Fig. 4.1. The $\mathrm{BS}$ set $\mathcal{B}=\left\{b_{1}, \ldots, b_{\mathrm{N}_{\mathrm{BS}}}\right\}$ consists of MBS set $\mathcal{M}=\left\{m_{1}, \ldots, m_{\mathrm{N}_{\mathrm{MBS}}}\right\}$ and $\mathrm{SBS}$ set $\mathcal{S}=\left\{s_{1}, \ldots, s_{\mathrm{N}_{\mathrm{SBS}}}\right\}(\mathcal{B}=\mathcal{M} \cup \mathcal{S})$. The UEs $\mathcal{K}=\left\{k_{1}, \ldots, k_{\mathrm{N}_{\mathrm{UE}}}\right\}$ are uniformly distributed over the entire area. For the simplicity, we assume that all of them use

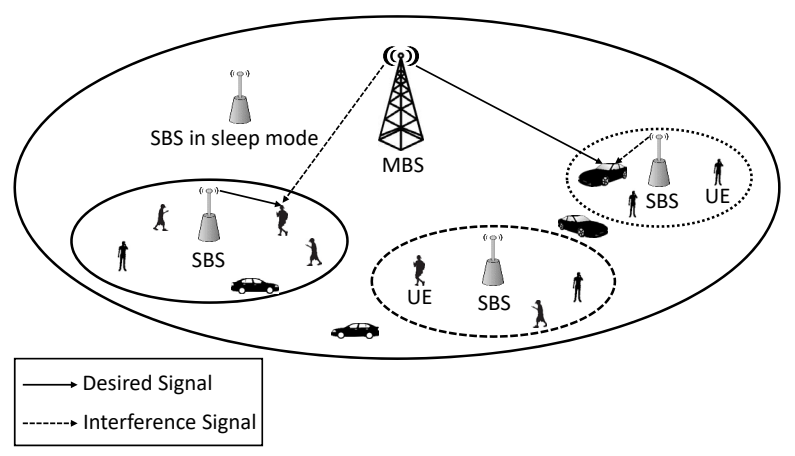

Figure 4.1: Two-tier HetNet with small cells and mobile users.

the same frequency band. We also consider that the UEs move in a random walk fashion, where at each time increment dt, and its velocity is expressed as follows

$$
\boldsymbol{v}_{\mathrm{t}}=\boldsymbol{v}_{\mathrm{t}-1} \rho+\sqrt{1-\rho^{2}} v_{\text {mean }} \boldsymbol{V}
$$

where $\rho=e^{\frac{-d t . a_{\text {mean }}}{v_{\text {mean }}}}$ represents the correlation of the velocity between time increments $a_{\text {mean }}$ and $v_{\text {mean }}$, which are mean acceleration and velocity, respectively. The magnitude of the velocity vector $\boldsymbol{V}$ is Rayleigh distributed.

If the UE $k$ is served by the BS $b \in \mathcal{B}$ whose downlink transmit power at time instant $t$ is given as $p_{b}(t)$, then the signal to interference plus noise ratio (SINR) experienced by the UE is given by 


$$
\gamma_{b}^{k}(x, t)=\frac{p_{b}(t) g_{b}^{k}(x, t)}{\sum_{b^{\prime} \neq b} p_{b^{\prime}}(t) g_{b^{\prime}}^{k}(x, t)+N_{0}},
$$

where $g_{b}^{k}(x, t)$ is the free space pathloss from the UE location $x$ to the BS, and $N_{0}$ is the noise power. The maximum throughput attained at the UE with bandwidth $B$ is given by the Shannon equation written as

$$
C_{\mathrm{k}}(x, t)=B \log _{2}\left(1+\gamma_{b}^{k}(x, t)\right)
$$

Further, we consider that UEs are guaranteed to achieve the constant bit rate $R_{\mathrm{k}}$ as a result of the load experienced by the BS, which can be expressed as

$$
\tau_{b}(t)=\sum_{k \in \mathcal{K}_{b}} \frac{R_{\mathrm{k}}}{C_{\mathrm{k}}(x, t)} .
$$

This determines the total fractional time required by the BS to deliver rate $R_{\mathrm{k}}$ for its associated users denoted as $\mathcal{K}_{b}$.

The power consumption model in [87] evaluates the total power needed by a BS to generate RF output power at its antenna elements and this can be expressed as

$$
P_{\text {total }}=\frac{P_{\mathrm{BB}}+P_{\mathrm{RF}}+P_{\mathrm{PA}}}{\left(1-\sigma_{\mathrm{DC})}\left(1-\sigma_{\mathrm{MS})}\left(1-\sigma_{\mathrm{cool}}\right)\right.\right.},
$$

where $P_{\mathrm{PA}}=\frac{P_{b}}{\eta\left(1-\sigma_{\text {feed }}\right)}$ is the power consumed by the power amplifier of efficiency $\eta$ to transmit RF output power $P_{b}$, while $P_{\mathrm{BB}}$ and $P_{\mathrm{RF}}$ are the powers consumed by base band and RF components of the BS, respectively. Parameters $\sigma_{\text {feed }}, \sigma_{\mathrm{MS}}$ and $\sigma_{\mathrm{DC}}$ denote the loss fractions of feeder, main supply and DC-DC power supply, respectively. The loss fraction of the cooling equipment $\sigma_{\text {cool }}$ will be zero for an SBS due to the absence of the cooling equipment. The BS can enter into the micro sleep mode by switching off its power amplifier in the case of low traffic load scenarios. The power consumption in the micro sleep mode can be written as

$$
P_{\text {sleep }}=\frac{P_{\mathrm{BB}}+P_{\mathrm{RF}}}{\left(1-\sigma_{\mathrm{DC})}\left(1-\sigma_{\mathrm{MS})}\left(1-\sigma_{\mathrm{cool}}\right)\right.\right.} .
$$


The energy efficiency can be improved, if the BS is able to autonomously adjust their transmission power $P_{b}$ based on the associated user traffic load in (4.4). In the following section, the BS power level switching problem is analyzed using the approach of game theory.

\subsection{Proposed Game Theoretic Approach}

A non-cooperative game $\mathcal{G}=\left(\mathcal{B}, \mathcal{A}_{b}, u_{b}\right)$, where the set of $\mathrm{BS}(\mathcal{B})$ are the players and each of them $b \in \mathcal{B}$ selects their action from the finite set of transmission power levels $\mathcal{A}_{b}$, is formulated in this section. The utility function of the $\mathrm{BS}$ is given by $u_{b}: \mathcal{A}_{b} \rightarrow \mathbb{R}^{-}$.

The set of BS action $\mathcal{A}_{b}=\left\{a_{b}^{(1)}, a_{b}^{(2)}, \ldots, a_{b}^{\left(\left|A_{b}\right|\right)}\right\}$ comprises of the action set of MBS $\mathcal{A}_{m \in \mathcal{M}}=\left\{0, P_{\max }\right\}$ and action set of $\operatorname{SBS} \mathcal{A}_{s \in \mathcal{S}}=\left\{0, \frac{P_{\max }}{3}, \frac{2 P_{\max }}{3}, P_{\max }\right\}$ where $\mathcal{A}_{b} \in \mathcal{A}_{\mathrm{m}} \cup \mathcal{A}_{\mathrm{s}}$. At each time instant, the BS $b \in \mathcal{B}$ selects its action $a_{b}(t)$ with a certain probability which forms the basis of the mixed strategy concept and it is given by

$$
\pi_{b}(t)=\mathbb{P}\left(a_{b}(t)=f_{b}\right)
$$

where $f_{b}$ is the outcome of a selected action by randomization device called roulette wheel. The main objective of the game is that each BS iteratively selects its best action which results in the highest utility.

In this paper, we consider the following multi-criteria utility function for handover decisions

$$
u_{b}(t)=-\omega \tilde{P}_{b}(t)-\phi \tilde{\tau}_{b}(t)-\psi \tilde{s}_{b}(t)
$$

where $\tilde{P}_{b}(t)$ is the power consumed by the BS in either active or sleep state given in (4.5) and (4.6), respectively, $\tilde{\tau}_{b}(t)$ is the BS load given in (4.4), $\tilde{s}_{b}=\frac{N_{\mathrm{PP}, b}(t)}{n_{b}(t)}$ represents the fraction of ping-pongs handovers ${ }^{1} N_{\mathrm{PP}, b}$ compared to total handovers $n_{b}(t)$, while

\footnotetext{
${ }^{1}$ We define ping-pong handover as a handover where a user equipment stays less than one second in a cell before making a new handover.
} 
$\omega, \phi, \psi$ represent their corresponding weights. It is desirable to reduce the number of ping-pong handovers in a network, since they trigger exchange of the coordination messages among the BSs (hence, resulting in higher background traffic), and the packets intended for the desired user may be lost during the frequent handovers [33].

The game $\mathcal{G}$ admits at least one equilibrium, since the action set $\mathcal{A}_{b}$ is discrete and finite. The outcome of this non-cooperative game results in suboptimal mixed strategy of Nash equilibrium. Therefore, other solution concepts, which achieve optimal expected payoff for a player, need to be obtained. Auman et al. showed in [88] that allowing the players to correlate their actions in non-cooperative games can achieve the equilibrium better than convex hull of the Nash equilibrium. For instance, if the signals are generated based on the common knowledge of the players' actions in a game, then the actions of the players, which are drawn from a distribution based on the generated signals, will result in a correlated equilibrium (CE). Here, the player is more likely to select an action which yields the best expected payoff conditioned on player seeing its own action.

We consider a slight variation of the CE scenario, where the player has the best expected payoff for an action before seeing the action itself. Such a distribution is called "coarse correlated equilibrium" defined as follows.

Definition 4.1.1 A coarse $C E$ is a probability distribution $\pi_{b}$ that has for every player $b \in \mathcal{B}$ and his every action $a_{b}^{\prime} \in \mathcal{A}_{b}$ :

$$
\sum_{a_{-\mathrm{b}}^{\prime} \in \mathcal{A}_{-b}}\left(u_{b}\left(a_{b}^{\prime}, \mathbf{a}_{-b}\right) \pi_{-b, \mathbf{a}_{-b}}\right)-\sum_{a \in \mathcal{A}_{b}}\left(u_{b}(a) \pi_{b, a}\right) \leq 0
$$

where $u_{b}(a)$ is the utility of the player when action $a$ is drawn from the distribution $\pi_{b}$ and $\pi_{-b, \mathbf{a}_{-b}}$ is the marginal distribution of a player $b$ action computed using the joint distribution of its action $a_{b}^{\prime}$ with other players' actions $a_{-b} \in \mathcal{A}_{-b}$ which is also expressed as 


$$
\pi_{-b, \mathbf{a}_{-b}}=\sum_{a_{b}^{\prime} \in \mathcal{A}_{b}} \pi\left(a_{b}^{\prime}, a_{-b}\right) .
$$

The empirical distribution of the play in the regret matching adaptive procedure converges to the CE distributions as time $t \rightarrow \infty$ [89]. For the finite time interval and any $\varepsilon>0$, it converges to a distance lesser than $\varepsilon$ from the CE. We follow this regret matching framework and for the finite time interval, the empirical distribution converges to $\varepsilon>0$ coarse correlated $\varepsilon$-equilibrium which is basically obtained by replacing the right hand side in (4.9) by $\varepsilon$. In the following section, we explain the proposed regret matching learning procedure to attain coarse correlated $\varepsilon$-equilibrium which yields optimal expected payoff for every player.

\subsubsection{Regret Based Game Theoretic Learning Scheme}

The basic idea of the regret based learning scheme is that the player evaluates the regret for not having played the action and aims at minimizing the regret by changing its actions over the time. Hence, the action played yields best expected utility. Let us assume the game $\mathcal{G}$ is repeatedly played at every time instant $t$ and the BSs are constantly changing their actions based on the outcome from their respective distribution $\pi_{b}(t)$ and observe the utility $u_{b}(t)$ which is defined in (4.8) and can simultaneously capture transmit power, load, and ping-pong handovers. The goal is to adapt the mixed strategy $\pi_{b}$ so that it minimizes the regret $r_{b}(t)$ over the time. Usually the regret evaluation needs to know the utility $u_{b}(t)$ and this requires the knowledge of the other BS actions due to the load term $\tilde{\tau}_{b}(t)$ in (4.8). However, this is not feasible in practice due to the distributed nature of BSs. Estimation also needs to be performed as follows [51]: 


$$
\begin{aligned}
& \tilde{u}_{b}^{(l)}(t+1)=\tilde{u}_{b}^{(l)}+\Lambda_{b}(t+1)\left(u_{b}^{(l)}(t)-\tilde{u}_{b}^{(l)}\right), \\
& \tilde{r}_{b}^{(l)}(t+1)=\tilde{r}_{b}^{(l)}+\Upsilon_{b}(t+1)\left(\tilde{u}_{b}^{(l)}-u_{b}^{(l)}(t)-\tilde{r}_{b}^{(l)}\right), \\
& \tilde{\pi}_{b}^{(l)}(t+1)=\tilde{\pi}_{b}^{(l)}+\Delta_{b}(t+1)\left(G_{b}^{l}\left(\tilde{r}_{b}^{(l)}(t+1)\right)-\tilde{\pi}_{b}^{(l)}\right),
\end{aligned}
$$

$\Lambda_{b}, \Upsilon_{b}$ and $\Delta_{b}$ are the learning rates for the utility, regret and mixed strategy probability, respectively. Generally, the learning rate follows the scheme $\left(\frac{1}{t}\right)^{e}$, where $e$ is the exponent of the learning rate similar to all BSs. The estimation of the mixed strategy $\pi_{b}^{l}(t)$ of actions is performed according to the Boltzmann-Gibbs (BG) distribution $G_{b}^{l}$ which weighs them relatively based on their regrets. Hence, highest regret has the maximum probability and the BSs are more likely to pick these actions through roulette wheel selection in (4.7). The BG distribution can be written as [51]

$$
G_{b}^{(l)}\left(\tilde{r}_{b}^{l}(t+1)\right)=\frac{\exp \left(\kappa_{b} \tilde{r}_{b}^{(l)}(t+1)\right)}{\sum_{l^{\prime} \in \mathcal{A}_{b}} \exp \left(\kappa_{b} \tilde{r}_{b}^{\left(l^{\prime}\right)}(t)\right)},
$$

where $\kappa_{b}>0$ is a temperature parameter which balances the exploitation of the actions with higher regrets by exploring the actions with lower regrets. In this way, the BS picks the best action with the evolution of time and its mixed strategy $\pi_{b}(t)$ converges to the coarse correlated $\varepsilon$-equilibrium.

The frequent change in the power levels of the regret matching learning scheme results in the increased signaling load when the handover decisions are made on a single metric such as the signal strength. Therefore, the multi-criteria handover decision schemes are necessary. In this paper, we propose the context-aware multi-criteria handover scheme to minimize the unnecessary handovers, which will be discussed further in the following section. 


\subsection{Context-Aware Fuzzy Handover Scheme}

The proposed fuzzy context-aware handover scheme contains two stages: i) handover necessity decision, and ii) target BS selection.

\subsubsection{Handover Necessity Decision}

In the first stage, the user determines the handover decision condition based on the handover factor determined by the multi-criteria fuzzy logic controllers (FLCs) as seen in Fig. 4.2. We consider SINR, throughput and BS load as given in eq. (4.2), eq. (4.3) and eq. (4.4), respectively. In addition to these parameters, UE-BS distance and velocity of the users are also taken into account to determine the handover decision condition. The fuzzy reasoning helps to deal with the imprecise nature of the parameters involved in the handover decision condition and also it is easy to interpret the influence of these multi-attribute parameters on the handover decision due to the usage of if-then rules.

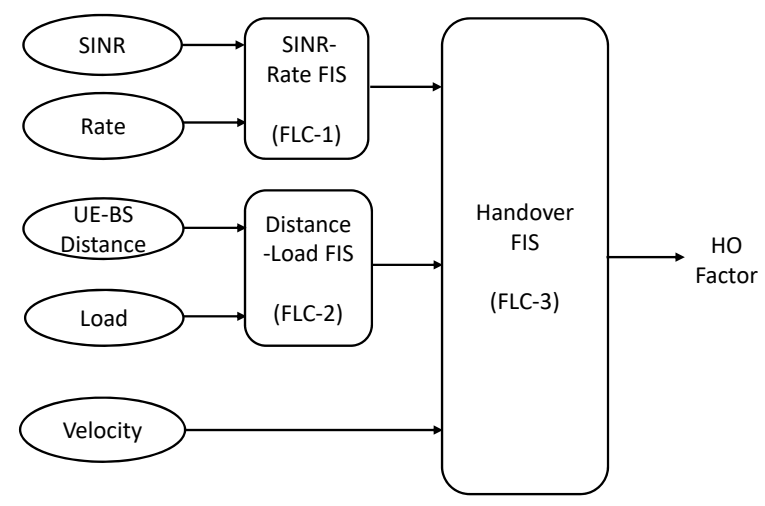

Figure 4.2: The proposed fuzzy logic controller for the handover decisions, composed of three fuzzy inference systems (FIS).

The fuzzy if-then rules maps the input to suitable output space. To reduce the number of if-then rules, the fuzzy logic controllers are connected in a parallel fashion. The SINR and rate parameters are passed to FLC-1 to obtain SINR-Rate factor; similarly the Distance-Load factor is obtained using FLC-2 as shown in Fig. 4.2. 
The output of these two FLCs together with the velocity parameter are fed to the handover FIS (FLC-3) to determine the handover factor. Next, we determine the impact of parallel combining fashion on the if-then rules reduction. For instance, if all five parameters having three fuzzy sets as low, medium and high directly fed to the handover FIS, then the number of if-then rules of the handover FIS will be $3^{5}=243$, which is reduced to $3^{3}=27$. This is due to the parallel combination of the handover context parameters in the FLC-1 and FLC-2. Usually, the fuzzy inference process in a FLC consists of several steps.

In the first step fuzzification of the inputs are performed, the crisp values at the input of FLC are fuzzified using a membership function, which is designed purely based on human intuition. To this end, triangular $h(x)$ and trapezoidal $p(x)$ membership functions are employed, and can be expressed as follows

$$
h(x)=\left\{\begin{array}{ll}
\frac{x-a}{b-a}, & a \leq x \leq b \\
\frac{c-x}{c-b}, & b \leq x \leq c
\end{array}, \quad p(x)= \begin{cases}\frac{x-l}{m-l}, & l \leq x \leq m \\
1, & m \leq x \leq n . \\
\frac{u-x}{u-n}, & n \leq x \leq u\end{cases}\right.
$$

The parameters $[a, b, c]$ and $[l, m, n, u]$ of the $h(x)$ and $p(x)$, respectively represent the bounds of the input space. The membership functions for the user velocity consists of three fuzzy sets namely low, medium and high as shown in Fig. 4.3. The low and high fuzzy sets are described by trapezoidal membership functions, while the medium fuzzy set uses the triangular membership function. It is important to notice that the membership functions are overlapping due to the smooth transition boundary which is an underlying characteristic of the fuzzy sets; i.e., the precise input values during fuzzification process can belong to more than one fuzzy set with the different degree of membership shown in Fig. 4.3. For instance, user velocity $30 \mathrm{~km} / \mathrm{hr}$ belongs to the low fuzzy set with a degree of 0.9 and to the medium fuzzy set with a degree of 0.25 . Hence, this might trigger several if-then rules as a result. 


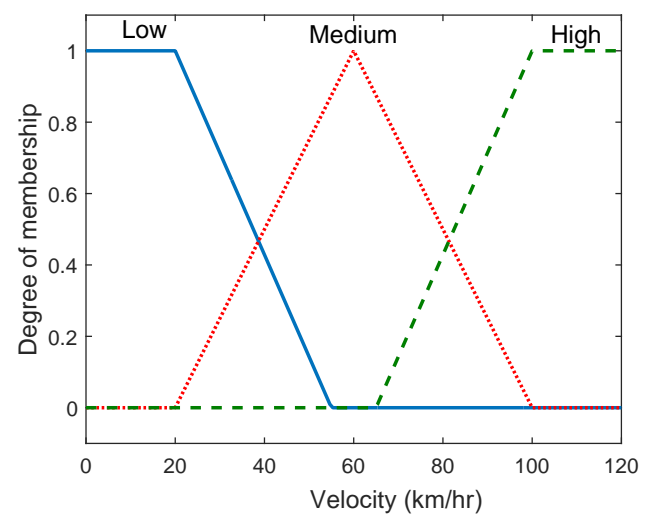

Figure 4.3: Membership functions for different user velocities.

In the second step, the if-then rules associated with the membership functions are identified and their respective firing strength is calculated. Suppose that one of the if-then rules of the Handover FIS shown in Fig. 4.2 is given as "If (Rate-SINR-factor is Low) and (Distance-Load-factor is Medium) and (Velocity is High) then (MBSHO-factor is Medium)", where AND logical operation is a simple arithmetic product and the firing strength for rule $i$ can be expressed as follows:

$$
\alpha_{i}=\mu_{\text {Low }}\left(x_{1}\right) \times \mu_{\text {Medium }}\left(x_{2}\right) \times \mu_{\text {High }}\left(x_{3}\right),
$$

where $\mu_{\text {Low }}\left(x_{1}\right), \mu_{\text {Medium }}\left(x_{2}\right)$, and $\mu_{\text {High }}\left(x_{3}\right)$ are the membership functions of the input rate-SINR-factor, distance-load-factor and the velocity, respectively. Similarly, implication of the if-then rule is performed by multiplying its firing strength $\alpha_{i}$ with the output membership functions to obtain the rule output. The output membership functions are either linear or constant, and therefore, we consider only Sugeno type fuzzy inference system $[90,91]$.

In the final step, defuzzification is carried out and the final precise output of the FLC is the weighted average of all the rule outputs, given as

$$
w=\frac{\sum_{i=1}^{N} \alpha_{i} z_{i}}{\sum_{i=1}^{N} \alpha_{i}},
$$



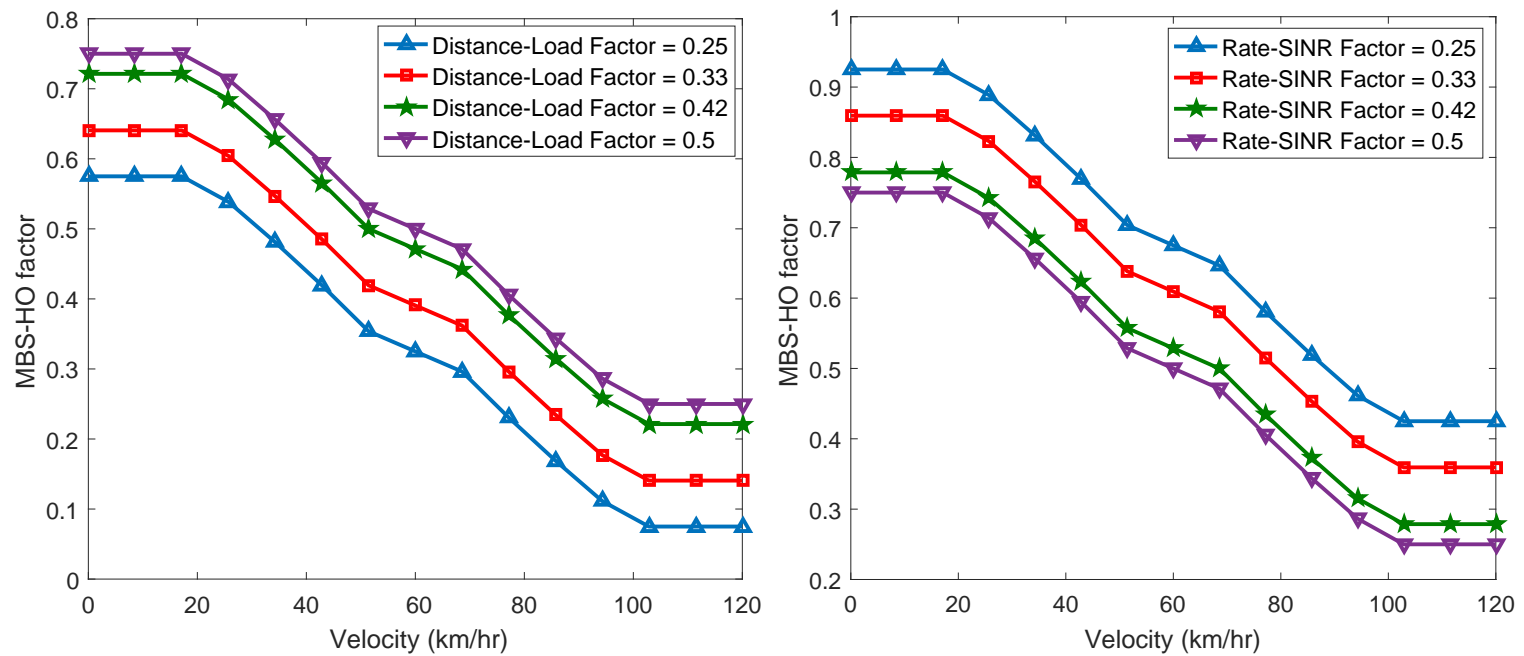

(a) Control surface for different distance-load(b) Control surface for different rate-SINR factors. factors.

Figure 4.4: Design of the handover FLC for the MBS.

where $z_{i}$ is the output membership value for the rule $i$. An illustration of the weighted average value $w$ for the Handover FIS in the case of MBS and SBS is shown in Figs. 4.4 and 4.5 , respectively. We observe that with increase in the velocity, the handover factor reduces for MBS, while it increases for SBS with respect to the proposed if-then rules for the Handover FIS shown in Fig. 4.2. This implies that a UE residing at MBS and traveling at higher velocity will have a reduced likelihood of a handover. However, it increases for a UE associated with an SBS. In Figs. 4.4(a) and 4.5(a), the handover factors are shown as the functions of the velocity in the different distance-load factors, while Figs. 4.4(b) and 4.5(b) show the handover factors under the different rate-SINR factors for the MBS and the SBS, respectively. We can see that the possibility of the handover increases as the distance-load factor increases, whereas it decreases with the increase in the rate-SINR factor. This implies that proposed if-then rules follow general trend on how the chances of the initiating handover varies with the parameters such as distance, rate, load and SINR. 

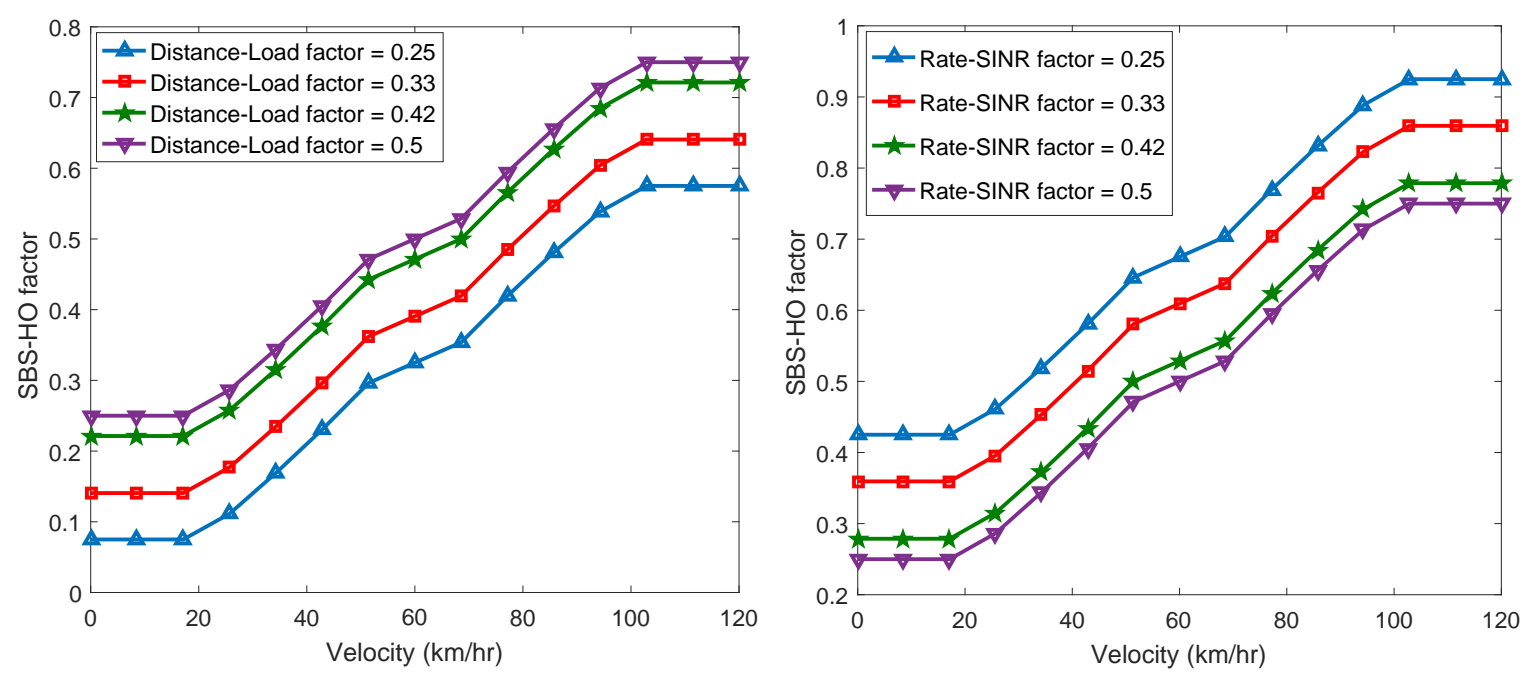

(a) Control surface for different distance-load(b) Control surface for different rate-SINR facfactors.

tors.

Figure 4.5: Design of the handover FLC for the SBS.

Once the HO factor is obtained, it is compared with the threshold to determine the handover decision condition. If the HO factor exceeds the threshold, a handover is initiated. The threshold should be carefully adjusted to prevent the unnecessary handovers among MBSs and SBSs.

\subsubsection{Target BS Selection}

The second stage of the proposed handover scheme is the target BS selection. We follow the multi attribute decision making (MADM) scheme called fuzzy technique for the order of preference by similarity to the ideal solution (FTOPSIS) explained in [68] for the BS selection. The overall proposed fuzzy handover scheme is summarized in Fig. 4.6. The BSs are ranked based on their own ranks, and the BS with highest rank is selected to make a handover. The proposed fuzzy handover scheme with handover necessity decision and target BS selection is summarized in Fig. 4.6.

\subsection{Simulation Results}

Our proposed context aware fuzzy handover scheme is evaluated using the rudimentary network emulator (RUNE) in Matlab simulation platform. We consider a simula- 


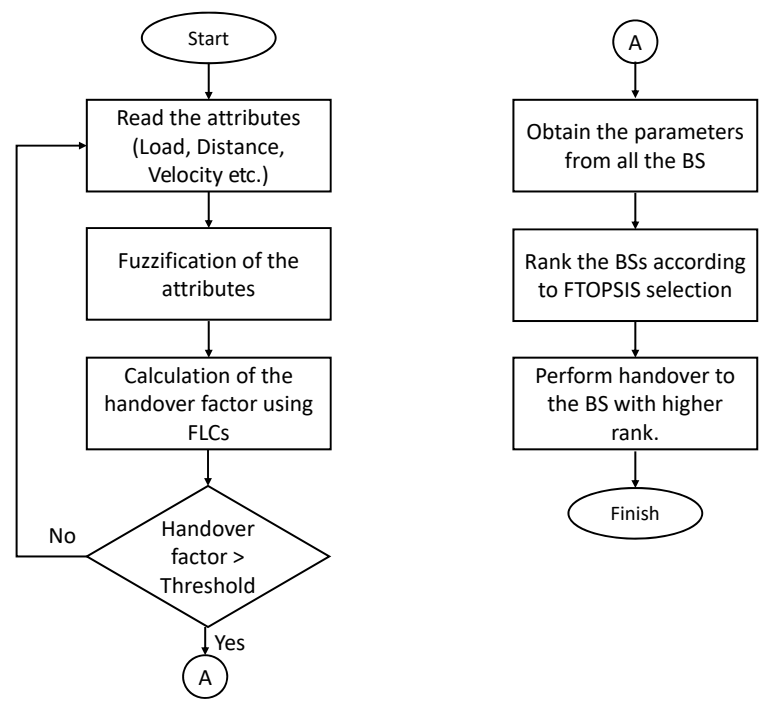

Figure 4.6: Proposed fuzzy logic handover scheme: handover necessity decision (left), and target BS selection (right).

tion scenario as seen in Fig. 4.7 with a single macrocell, as well as multiple SBSs/UEs uniformly distributed over the geographical area. Unless specified, key simulations parameters are as given in Table 4.1.

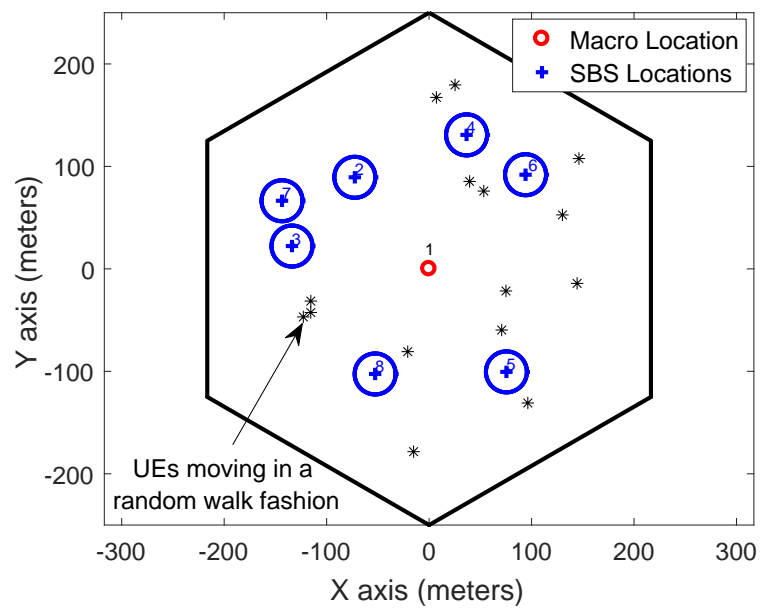

Figure 4.7: Two tier HetNet where MBS is located at the origin and circles represent the coverage of the SBSs.

The BSs switch their transmission power levels based on the regret learning scheme shown in Section 4.1.1 and it is worth mentioning that we do not consider wake-up mechanism for the BS. Therefore we assume that there is no delay when it wakes 
Table 4.1: Simulation parameters.

\begin{tabular}{|l|l|l|}
\hline Parameter & MBS & PBS \\
\hline Cell radius & $250 \mathrm{~m}$ & $20 \mathrm{~m}$ \\
\hline Number of cells & $1\left(\mathrm{~N}_{\mathrm{MBS}}\right)$ & $7\left(\mathrm{~N}_{\mathrm{SBS}}\right)$ \\
\hline Minimum distance & $75 \mathrm{~m}$ for MBS-SBS & $40 \mathrm{~m}$ for SBS-SBS \\
\hline Minimum load & $35 \mathrm{~m}$ for MBS-UE & $10 \mathrm{~m}$ for PBS-UE \\
\hline Num. power strategies & 2 & 0.1 \\
\hline Maximum TX power & $16 \mathrm{dBm}$ & 4 \\
\hline \multicolumn{2}{|c|}{ System Paramters } \\
\hline Packet arrival rate & $0 \mathrm{dBm}$ \\
\hline Mean packet size & $1 \mathrm{kbps}$ \\
\hline Channel bandwidth $(B)$ & $1800 \mathrm{bits}$ \\
\hline Number of users $\left(N_{\mathrm{UE}}\right)$ & $10 \mathrm{MHz}$ \\
\hline Time interval between iterations & 15 \\
\hline
\end{tabular}

up from the sleep mode. We study how the user mobility influences various BS performance parameters such as the energy consumption, the ping-pong rate, and the offered throughput, separately in the following sections.

\subsubsection{Impact on BS Energy Consumption}

The SBS energy consumption versus time is evaluated for user velocities $v=\{30,60,120\}$ $\mathrm{km} / \mathrm{hr}$ and is shown in Fig. 4.8. We can see that the BS optimizes its energy consumption with time through the proposed regret learning scheme. The energy consumption is the lowest for the high velocity users, since the users are served by the MBS and handovers are not triggered by the FLC as implied by Fig. 4.2. As a result, the SBSs go into sleep mode which decreases the energy consumption, with a downside that it increases the load on the MBS. In the case of lower velocity users, handovers are more likely to be triggered to the SBS due to the velocity attribute considered in the fuzzy reasoning of the FLC in Fig. 4.2, which rejects the handover to the MBS. Therefore, more SBSs are active and this in turn increases the energy consumption. 


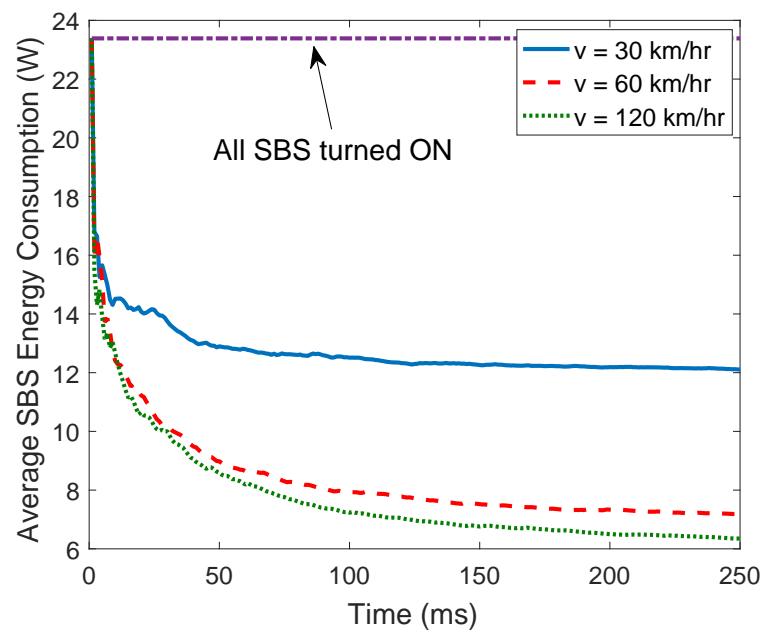

Figure 4.8: Energy consumption versus time (15 UEs).

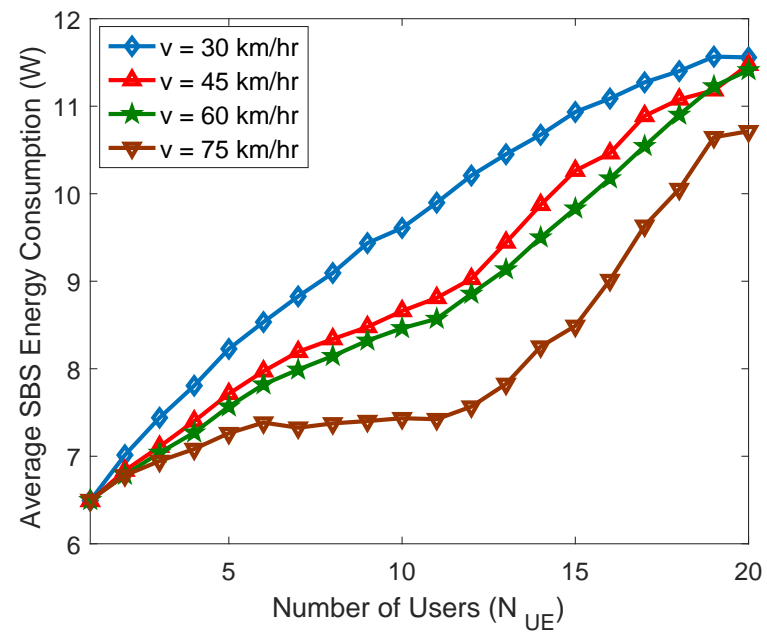

Figure 4.9: Average SBS energy consumption versus number of users $\left(\mathrm{N}_{\mathrm{SBS}}=7\right)$.

In Fig. 4.9, considering that the energy consumption reaches a steady state after some time (e.g., as shown in Fig. 4.8), we plot the average SBS energy consumption as a function of number of users in the network considering different velocities and using our proposed handover mechanism in Fig. 4.2. We can observe that when the user velocity is highest at $75 \mathrm{~km} / \mathrm{hr}$, the SBS energy consumption is minimized, since more users are kept at macrocell. On the other hand, for lower velocities, average SBS energy consumption is gradually increased, since more users are served by the SBSs. Moreover, when the number of users is increased, the SBSs also move 
into active mode to serve those users, hence increasing further the overall energy consumption. To support the results in Fig. 4.9, we further plot the average SBS load as a function of number of users in Fig. 4.10, which show a similar behavior with the energy consumption results in Fig. 4.9.

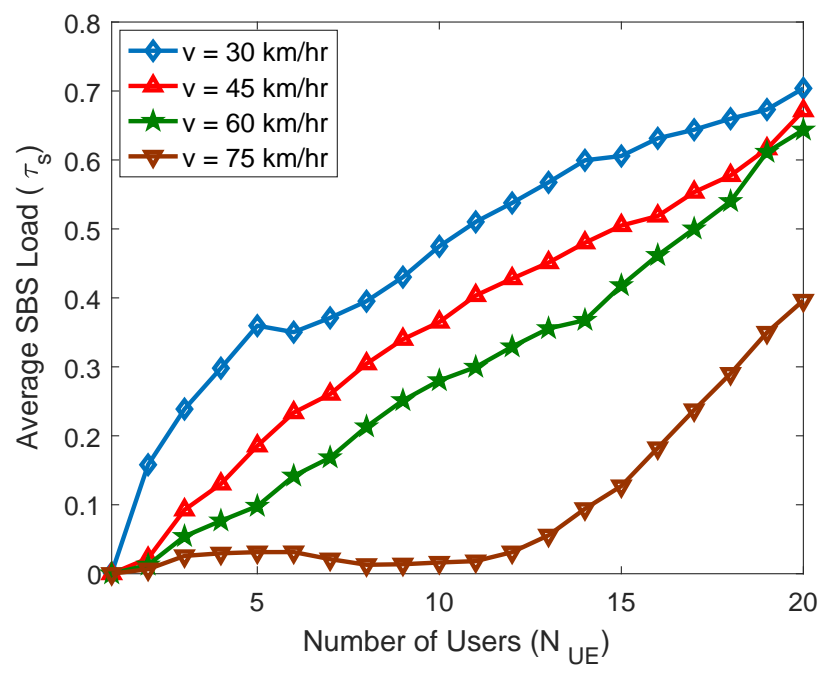

Figure 4.10: Average SBS load as a function of the number of users $\left(\mathrm{N}_{\mathrm{SBS}}=7\right)$.

\subsubsection{Impact on Ping Pong Performance}

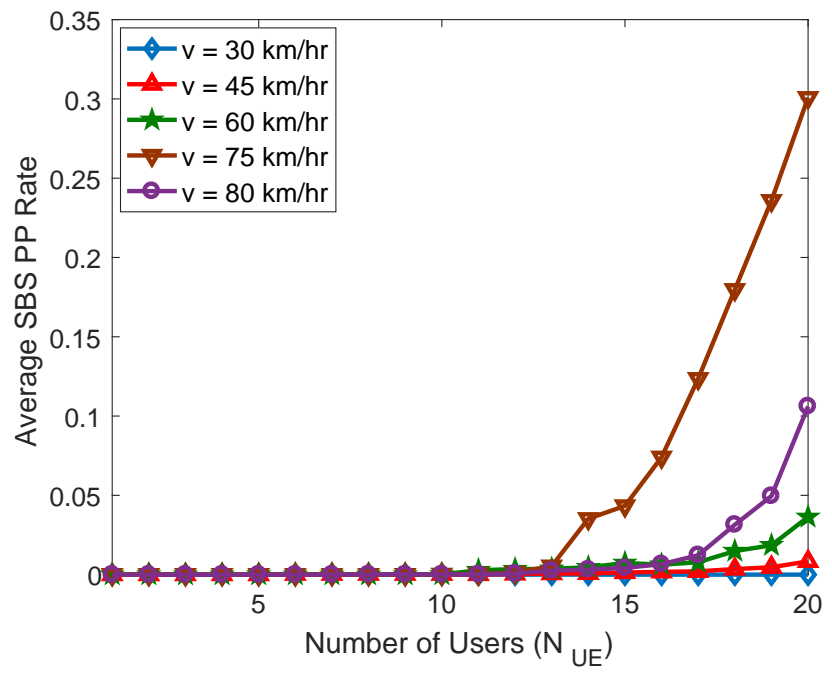

Figure 4.11: Average ping-pong handover rate as a function of the number of users $\left(\mathrm{N}_{\mathrm{SBS}}=7\right)$. 
The average ping-pong handover rate as a function of number of users is plotted and shown in Fig. 4.11. We observe that when the users have a velocity of $30 \mathrm{~km} / \mathrm{hr}$, there are no ping-pongs observed regardless of the number of users. For higher velocities, ping-pong handovers are observed. The ping-pong handover rate increases with user count, since the number of users also increase the load in the cells, which impacts the utility function in (4.8) and hence triggers handovers. We observe that the ping-pong rate is the highest for user velocity of $75 \mathrm{~km} / \mathrm{hr}$, rather than $80 \mathrm{~km} / \mathrm{hr}$. This is due to the handover decision framework discussed in Section 4.2, where high velocity users are inclined to remain at the MBS, which tends to reduce ping-pong handovers. In order to validate this observation, ping-pong rate is plotted as a function of user velocity for different $\mathrm{N}_{\mathrm{SBS}}$ in Fig. 4.12, which we observe to be aligned with the results in Fig. 4.11. In addition, we observe that ping-pong rate increases with $\mathrm{N}_{\mathrm{SBS}}$, since it becomes more likely to have handovers among neighboring SBSs. On the other hand, for user velocities higher than $100 \mathrm{~km} / \mathrm{hr}$, ping-pong rate sharply drops for $N_{\mathrm{SBS}}=20$, since many of the users are kept at the MBS, and the SBSs are placed into sleep mode.

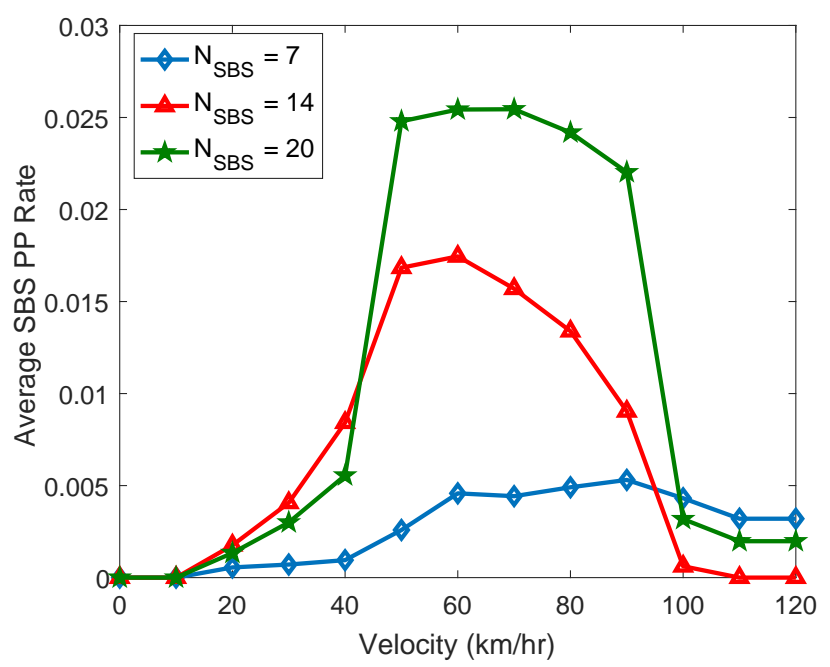

Figure 4.12: Average ping-pong handover rate as a function of user velocity (15 UEs). 


\subsubsection{Impact on BS Throughput Performance}

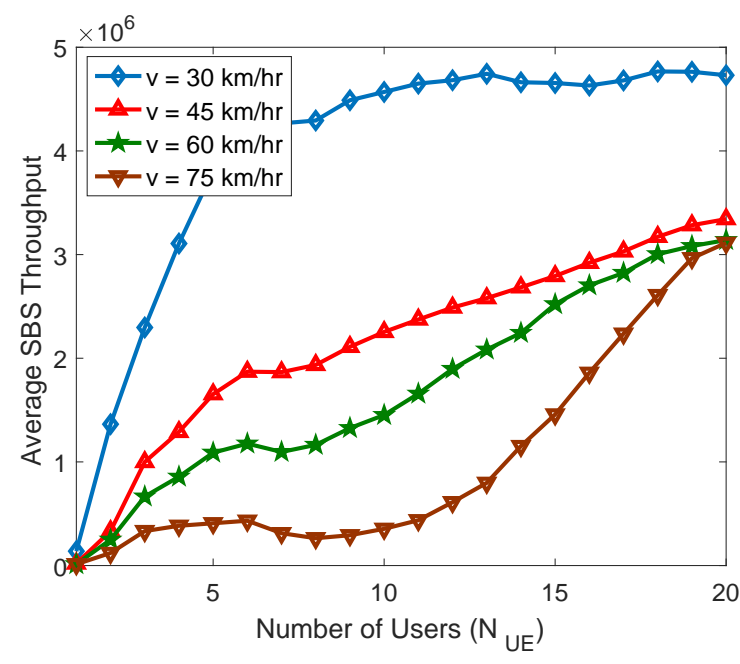

(a) Average SBS throughput as a function of number of users $\left(\mathrm{N}_{\mathrm{SBS}}=7\right)$.

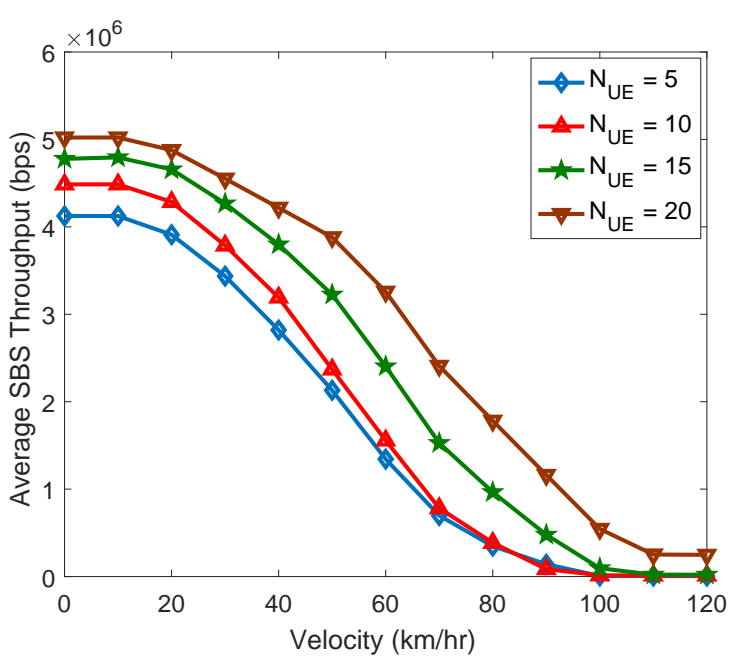

(b) Average SBS throughput as a function of user velocity $\left(\mathrm{N}_{\mathrm{SBS}}=15\right)$.

Figure 4.13: SBS throughput results.

The average SBS throughput as a function of number of users for user velocities $v=\{30,45,60,75\} \mathrm{km} / \mathrm{hr}$ is shown in Fig. 4.13a. We observe that the throughput per SBS is lower for higher user velocities, since the users are inclined to stay at the MBS. For lower velocities, the throughput is increased as a result of more users associating with small cells. Furthermore, we plot the average SBS throughput as a function of user velocity in Fig. 4.13b for $15 \mathrm{SBSs}$ and with different $\mathrm{N}_{\mathrm{UE}}$, which is aligned with the observations in Fig. 4.13a. We also observe that the average throughput is maximum for $\mathrm{N}_{\mathrm{UE}}=20$ users, but for higher velocities, throughput sharply reduces to similar values for all scenarios, since only small number of users are served at the SBSs. 
CHAPTER 5

\section{AN EXPERIMENTAL STUDY ON MOBILITY PERFORMANCE IN REAL LTE SCENARIOS USING SOFTWARE DEFINED RADIO}

\section{$5.1 \quad$ Introduction}

The present LTE systems has adopted hard handover mechanism, where the user equipment (UE) ongoing connection from source cell is broken before making a handover to the target cell. In the dense network and high speed user scenarios, this interruption can have serious implications on the delay sensitive traffic like voice, live streaming video etc. Therefore operators perform drive test and obtain network quality information in order to find any problems affecting the quality of service (QoS) of the users.

In recent years, SDR has gained much attention due to its ability to implement hardware components of a wireless communication system on a software platform. The simplest SDR transceiver consists of analog to digital (A/D) chip connected to an antenna in the receive path and digital to analog (D/A) chip connected to an antenna in the transmit path, which forms the RF front end. The signal processing stages are carried out in the digital domain by a personal computer.

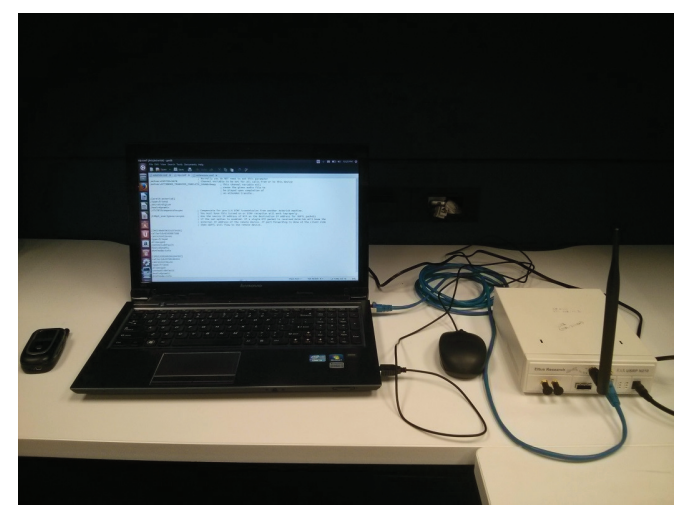

Figure 5.1: USRP 
One such SDR equipment is the universal software radio peripheral (USRP) shown in Fig. 5.1 which has become very popular due to low cost hardware and open ended platform for prototyping from basic to complex wireless systems [92]. It includes an RF front end (also referred as a daugtherboard) where all RF analog operations take place, and the digital signal processing tasks are carried out by the FPGA. The daughterboard determines the operational frequency range of the USRP. This makes it flexible and prototyping platform, where the performance of the communication protocols can be studied. In conventional drive test, the equipments used are expensive and are designed to carry out specific functions. Therefore, USRPs addresses this issues and can be best fit replacement for the conventional drive test equipment.

Drive test usually involves a vehicle carrying a equipment to record network measurements and a global positioning system (GPS) receiver to obtain geographical location information of the recorded data. The following section explains the measurement setup involved in drive test.

\subsection{Measurement Setup for signal recording in the drive test}

The measurements are obtained using a universal software defined radio (USRP) connected to laptop through a gigabit interface. The USRP features a integrated GPS disciplined oscillator (GPSDO) which helps to obtain the latitude and longitude coordinates, where the measurement was recorded. The USRP and laptop are powered through a uninterruptible power supply (UPS) lead acid batteries in the drive test. We use VERT900 antenna to scan the LTE frequencies in the range 730-2200 MHz and $3 \mathrm{~V}$ active antenna for the GPSDO to aid faster locking on to GPS constellation satellites.

The signal recording is performed in the LabVIEW (LV) platform and the block diagram code for recording the LTE measurements in LV is shown in Fig. 5.2. First 


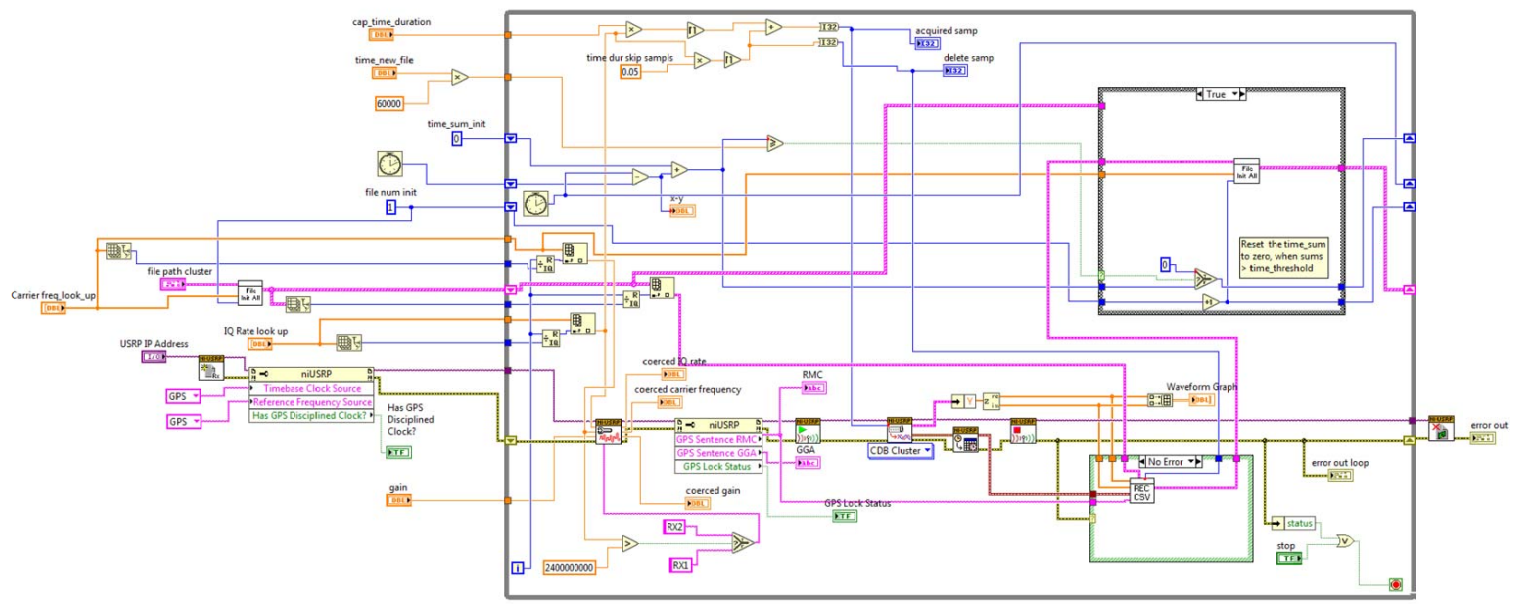

Figure 5.2: Signal recording block diagram in LabVIEW.

the GPS location information is obtained before initiating the measurement recording of the LTE signal. It can be extracted from the national marine electronics association (NMEA) information which is performed by the on-board receiver on the GPSDO. The NI-USRP driver helps to obtain NMEA sentences consisting of fix information (GGA) and recommended minimum data (RMC). The data format of the RMC is shown in Fig. 5.3

\begin{tabular}{|c|c|c|c|c|c|c|c|c|c|c|c|c|c|c|}
\hline $\begin{array}{l}\text { Msg } \\
\text { ID }\end{array}$ & \begin{tabular}{|l} 
UTC \\
Time
\end{tabular} & t & Lat & $\begin{array}{r}\mathrm{N} / \mathrm{S} \\
\text { Indicat }\end{array}$ & & & $\begin{array}{c}\mathrm{E} / \mathrm{W} \\
\text { Indicato }\end{array}$ & & \begin{tabular}{l|l} 
ed & Cou \\
er & Ov
\end{tabular} & \begin{tabular}{c|} 
Course \\
Over
\end{tabular} & Dat & $\mid \begin{array}{l}M \\
V\end{array}$ & $\begin{array}{l}\mathrm{Mag} \\
\mathrm{Var}\end{array}$ & 10 \\
\hline
\end{tabular}

Figure 5.3: RMC data format

For instance, the data field values in the RMC sentence are separated by comma and it may look like

\$GPRMC,220312.00,A,3546.3330,N,07840.4268,W,0.0,0.0,220617,,*25

where, \$GPRMC is the RMC protocol header which consists of the message ID, 220312.00 is the UTC time of format hours:minutes:seconds.fractional second, A is the status ( $\mathrm{A}=$ data valid; $\mathrm{V}=$ data not valid) and the format of the data field 220617 is ddmmyy and the empty fields are the magnetic variation and mode which is applicable to NMEA version 2.3 [93]. The sign convention is applied to the lati- 
tude and longitude based on the East/West and North/South indicators, where West and South are considered negative coordinates and it is recorded in a comma separated values (CSV) file after performing some manipulations. Further the LTE signal recording is performed and basically at every loop iteration the location information and LTE signal measurements are logged on to CSV spreadsheet file as shown in Fig. 5.2.

The LTE frequency bands are given in [94,95], and the active LTE frequencies can be determined whether the successful decoding of master information block (MIB) is achieved or not. The active LTE frequencies are then listed with their corresponding sampling rates and a lookup table is created. Next we perform the drive test and at every location the USRP carrier frequency and sampling rate are hopped based on the lookup table entries and the signal measurements are recorded in a separate file for each frequency. The elapsed time after recorded measurement at every loop iteration is added as shown in Fig. 5.2 and when this sum is greater than the threshold for a new file given by time_new_file, then new set of files are generated for GPS location, time, and lte measurements. The recorded measurements are then post-processed in the matlab which is explained in the following section.

\subsection{Post Processing of Drive Test Measurements in Matlab}

The main goal of the post processing is to obtain valid LTE base station information from the recorded measurements performed in the drive test.

Once the signal is recorded using Labview, MATLAB is used to process the data offline. In this section we will go through the the processing done to decode the Master Information Block (MIB). The blocks that we employ for MIB decoding are shown in Fig. 5.4. 


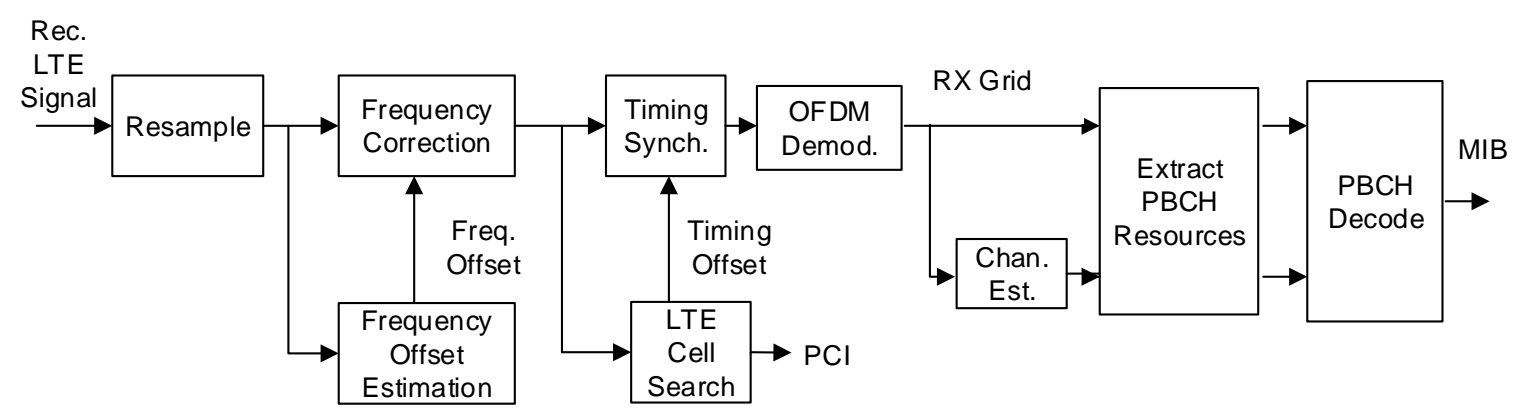

Figure 5.4: The recorded signal using Labview are processed in Matlab to obtain MIB bits. After resampling to $1.92 \mathrm{MHz}$ frequency and timing synchronization is performed, the LTE grid is constructed and $\mathrm{PBCH}$ symbols are decoded to obtain MIB bits.

\subsubsection{Resampling}

MIB is carried in Physical Broadcast Channel $(\mathrm{PBCH})$ which is contained in central 6 resource blocks of the LTE downlink signal. Therefore, the sampling rate required for MIB decoding is equal to $1.92 \mathrm{MHz}$. The USRP N210 can not sample at this rate therefore a resampling is required. The spectrum of the LTE signal centered at 739 MHZ and resampled signal using sampling rate of $1.92 \mathrm{MHz}$ are shown in Fig. 5.6 and Fig. 5.7 respectively. Given the sampling rate one may ask how many samples are required for MIB decoding. MIB is transmitted in the first subframe of each LTE radio frame every $10 \mathrm{~ms}$ and repeated 4 times until the next MIB is transmitted as shown in Fig. 5.5. When the signal reception is good 11 milliseconds of signal recording is adequate for MIB decoding.

\subsubsection{Frequency Offset Estimation and Correction}

After resampling, the frequency offset between the LTE basestation transmitter and USRP receiver is estimated and corrected. The estimation is based on cyclic prefix (CP) present in LTE downlink signal. To understand frequency offset estimation, let us consider one slot which lasts 0.5 milliseconds. A slot is half subframe and contains $1.92 \mathrm{MHz} \times 0.5 \mathrm{~ms}=960$ samples. In Frequency Division Duplex $($ FDD $)$ mode of LTE 


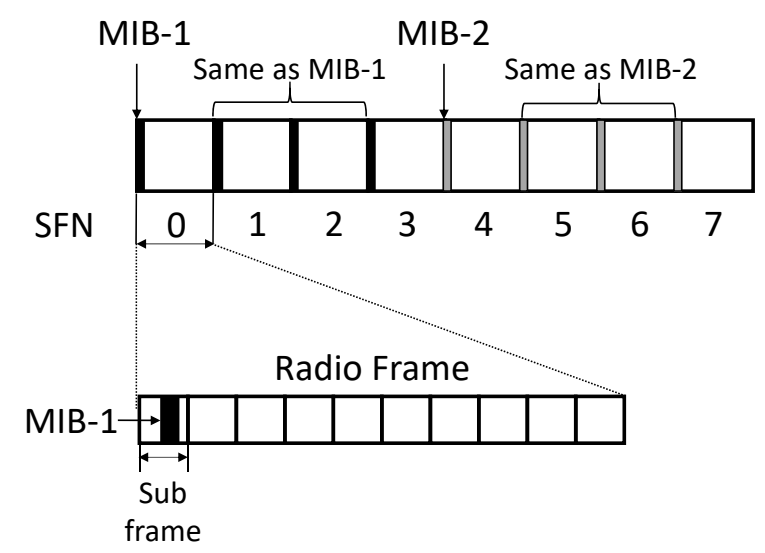

Figure 5.5: Scheduling of MIB information in time domain.

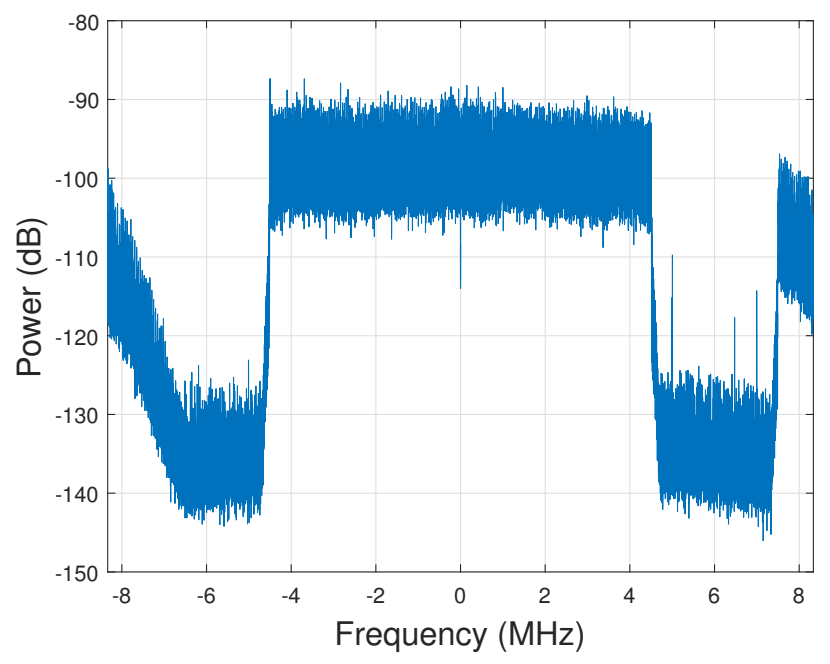

Figure 5.6: The spectrum of the LTE signal with $739 \mathrm{MHz}$ carrier frequency sampled at 16.67 MHz using USRP N210 in Labview.

where normal cyclic prefix is used, one slot contains 7 OFDM symbols. The FFT size for OFDM is 128 and 9 samples are used as CP except for the first symbol where CP length is 10 samples. Suppose $x(n)$ is the resampled signal and the sample at $n=n_{1}$ is part of the CP, then ignoring channel and AWGN,

$$
x\left(n_{1}+128\right)=x\left(n_{1}\right) \mathrm{e}^{j \phi 128}
$$

where $\phi$ is the angle created in one sample due to the frequency offset between the transmitter and the receiver. Note that,

$$
-\arg \left(x\left(n_{1}\right) x^{*}\left(n_{1}+128\right)\right)=128 \phi
$$




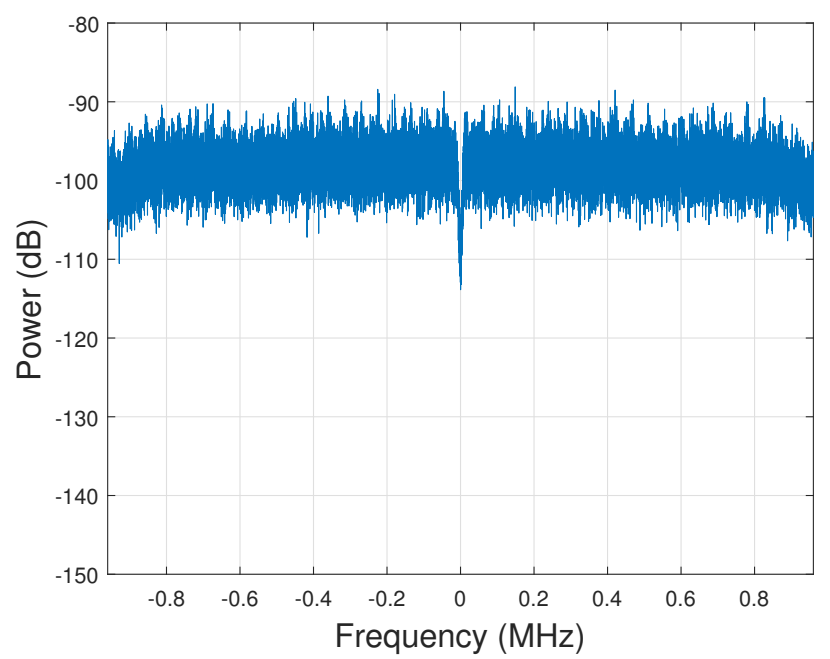

Figure 5.7: The spectrum of the resampled LTE signal with $1.92 \mathrm{MHz}$ sampling rate for MIB decoding. Decoding the central 6 resource blocks is possib;e with this sampling rate.

and frequency offset is related to $\phi$ by

$$
\hat{f}_{\text {offset }}=\frac{\phi}{2 \pi T_{s}}
$$

where $T_{s}=\frac{1}{1.92 \times 10^{6}}$ seconds.

In order to do frequency offset estimation we need to find the locations of the CP. Then (5.3) gives an estimate for each location. These estimates are then averaged to combat the effects of channel and the noise. Fig. 5.8 shows the plot of $y(n)=$ $x(n) x^{*}(n+128)$ averaged over all available slots in the received data. In this plot the peaks are the CP locations. Note that, this plot contains 960 samples which is the duration of one slot and there are 7 distinct peak locations corresponding to 7 OFDM symbols in a slot. This plot is also a good indication that the received signal is indeed an LTE signal.

Once the frequency offset is estimated it is easy to compensate it by

$$
y(n)=x(n) \mathrm{e}^{j 2 \pi \hat{f}_{\text {offset }} n T_{s}} .
$$

The maximum frequency offset that can be estimated using this technique can be calculated as follows: The angle in (5.2) should satisfy $|128 \phi| \leq \pi \Longrightarrow|\phi| \leq \frac{\pi}{128}$. 


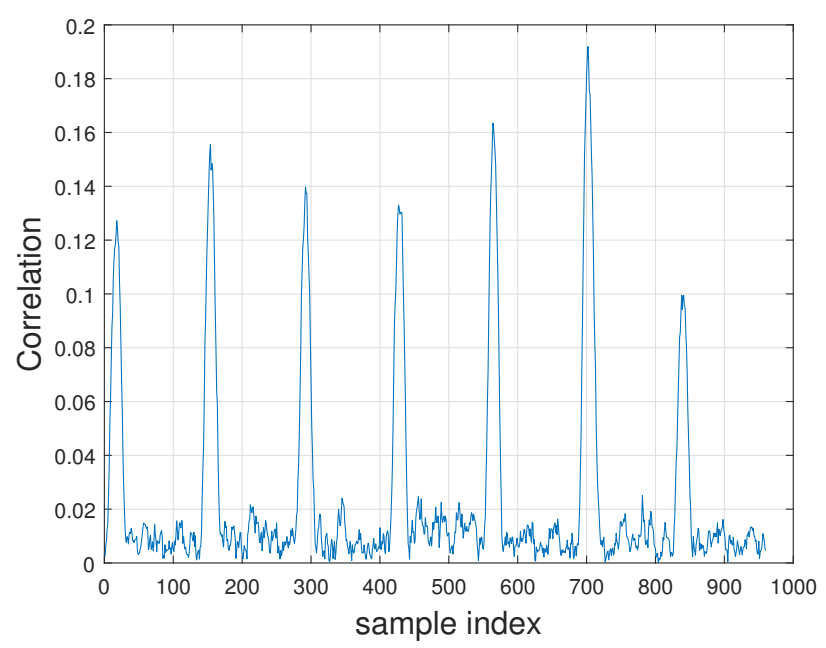

Figure 5.8: The product of the resampled signal and its 128 sample shifted version averaged over all available slots in the received signal. The peaks occur at locations locations of CP and these points are used for frequency offset estimation.

Therefore,

$$
\hat{f}_{\text {offset }} \leq \frac{\pi / 128}{2 \pi T_{s}}=\frac{1.92 \times 10^{6}}{2 \times 128}=7.5 \mathrm{kHz}
$$

which is half the subcarrier spacing.

\subsubsection{LTE Cell Search and Timing Synchronization}

In LTE system there are 504 unique physical cell IDs (PCI). The recorded LTE signal might contain signals originating from multiple eNodeB's with different PCIs. The PCI is determined using Primary Synchronization Signal (PSS) and Secondary Synchronization Signal (SSS). There are 3 available PSS signals and 168 available SSS signals. Each PCI has a unique combination of PSS and SSS signal. (Note that $3 \times 168=504)$. In order to find PCI all 504 combinations of PSS and SSS can be correlated with all the delayed versions of the signal. The PSS, SSS, and delay combination which gives the highest correlation provides an estimate of the PCI as well as the timing offset. As an example Fig. 5.9 shows the magnutude of the PSS and SSS signal when PCI=0 for the duration of 1 frame which is 19200 
samples. In this plot PSS appear in the 7th OFDM symbol of the subframe number 0 and subframe number 5 and SSS appear in the 6th OFDM symbol of the subframe number 0 and subframe number 5 . The SSS signal in subframe 0 is different than the one in subframe 5 which makes it possible to do estimation of the timing offset to subframe 0 .

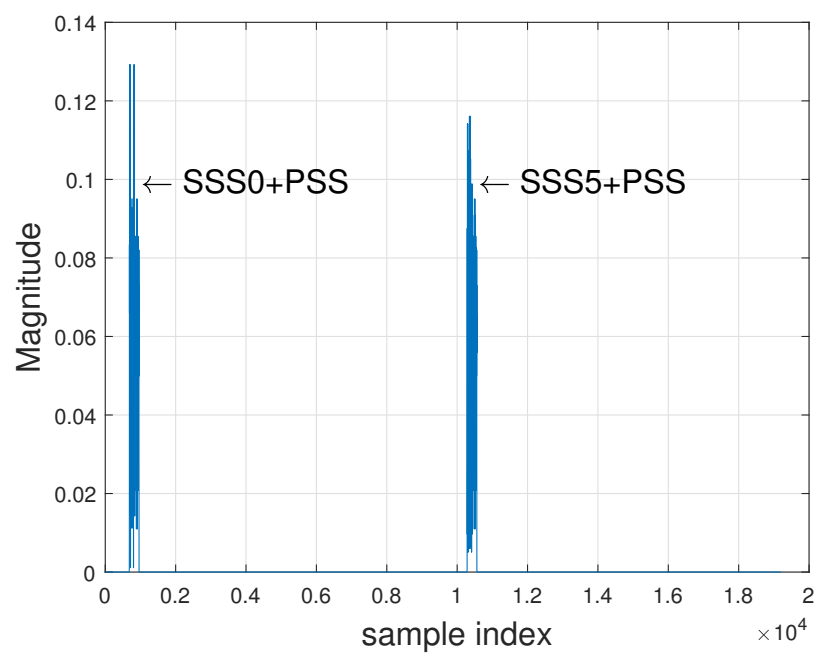

Figure 5.9: The magnitude of PSS and SSS signal for PCI=0. In order to find the PCI of the received signal SSS and PSS for all PCIs can be generated and correlated with the received signal. The PSS, SSS, and delay combination that gives maximum correlation is chosen for possible PCI candidate.

An example correlation is shown in Fig. 5.10. In this case maximum correlation is obtained for PCI equal to 134 and timing offset equal to 10306. The estimated offset value provides us with the first sample of subframe 0 .

\subsubsection{OFDM Demodulation}

Once the frequency and timing synchronization is completed OFDM demodulation can be performed. Here first the CP is removed and 128 point IFFT is performed and finally unused subcarriers are discarded to obtain the received grid containing 72 subcarriers. The result is stored in a matrix that contains 72 rows. The number of columns depends on the duration of the recorded signal. Fig. 5.11 shows the 


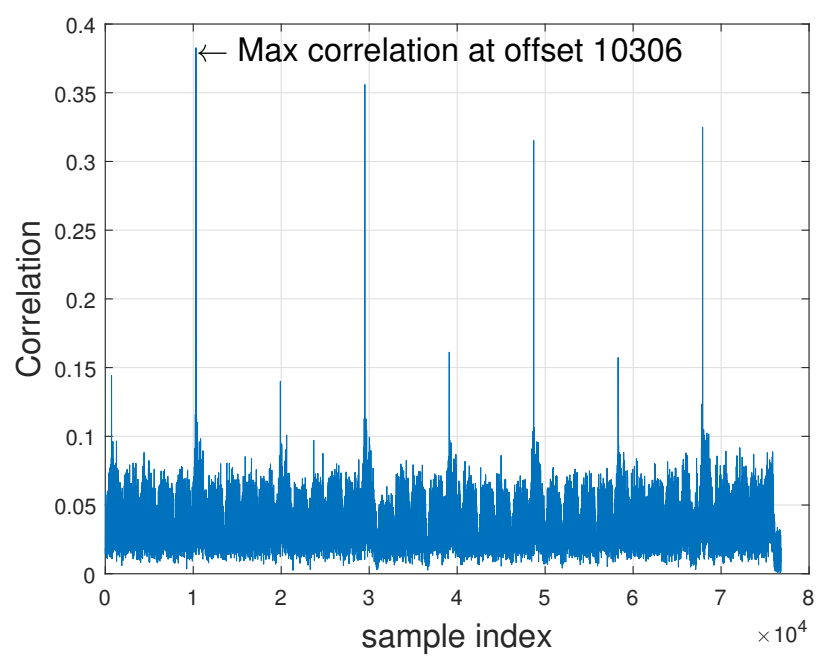

Figure 5.10: The PSS and SSS signal when PCI=134 gives maximum correlation of 0.38 at delay 10306. For the received sinal this is the maximum correlation value among all PSS, SSS, and delay combinations.

magnitude of the received grid for 14 symbols of subframe 0 . The locations of PSS, SSS, as well as PBCH are indicated on the plot.

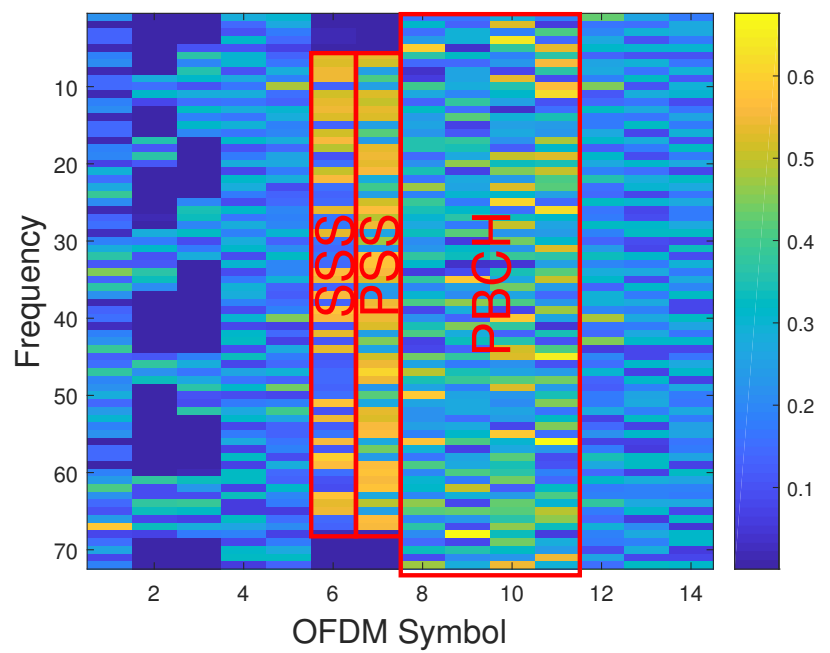

Figure 5.11: Received grid after OFDM demodulation. For the central 6 resource block, the grid contains 72 frequency bins. Here the grid is shown for the first subframe containing 14 OFDM symbols. The locations of PSS, SSS, and PBCH are also shown. 


\subsubsection{Channel Estimation}

Some specific locations in the received grid are reserved for cell specific reference signal (CRS) transmitted from each base station. Fig. 5.12a and Fig. 5.12b show the locations of these CRS from TX1 and TX2, respectively, for our received grid assuming number of transmitter antennas is 2 .
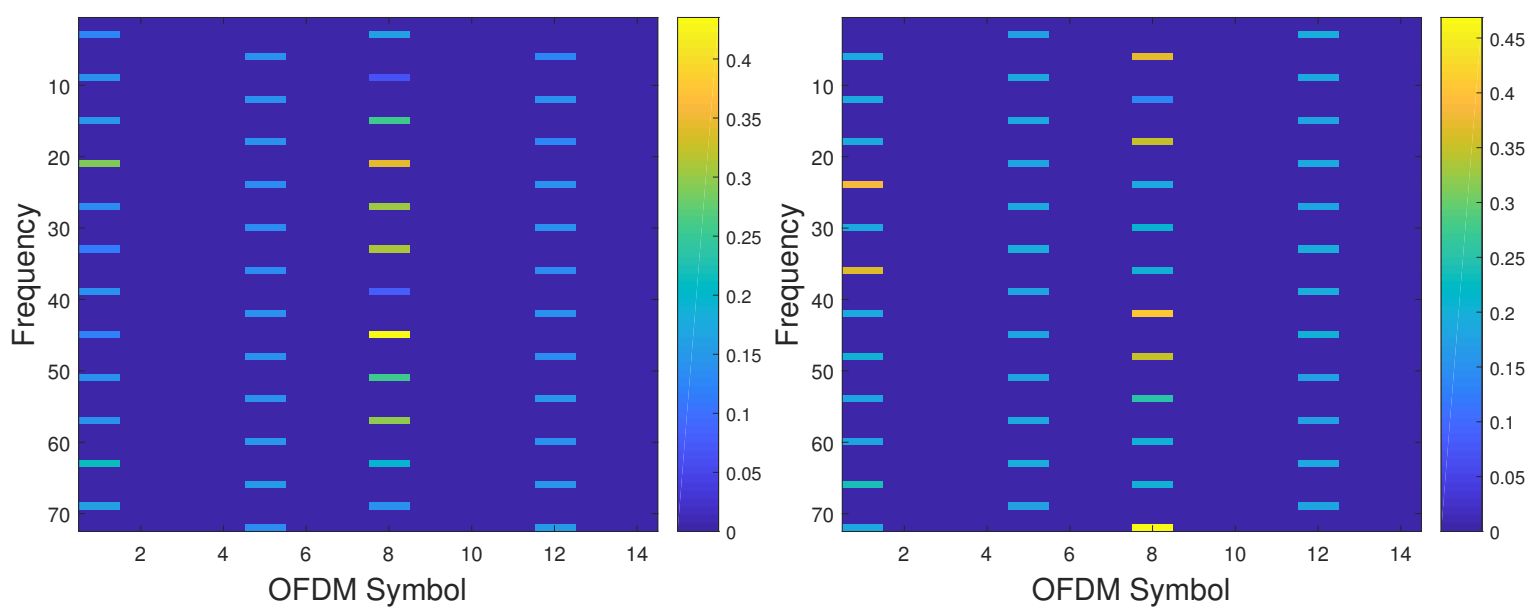

(a) The locations and magnitudes of CRS symbols from(b) The locations and magnitudes of CRS symbols from transmit antenna 1 . transmit antenna 2 .

Figure 5.12: CRS symbols from transmit antenna

The transmitted symbols at these locations are known at the receiver and channel estimates at these locations are interpolated to find channel estimates at other locations of the received grid. For each element of received grid there are $n_{R} \times n_{T}$ channel coefficients where $n_{R}$ and $n_{T}$ are number of RX and TX antennas, respectively. At this point the number of transmit antennas is not known. It is determined by trial and errror during PBCH decoding stage. Fig. 5.13 and Fig. 5.14 show the magnitude of the channel estimates obtained for our received grid from the two transmitters 


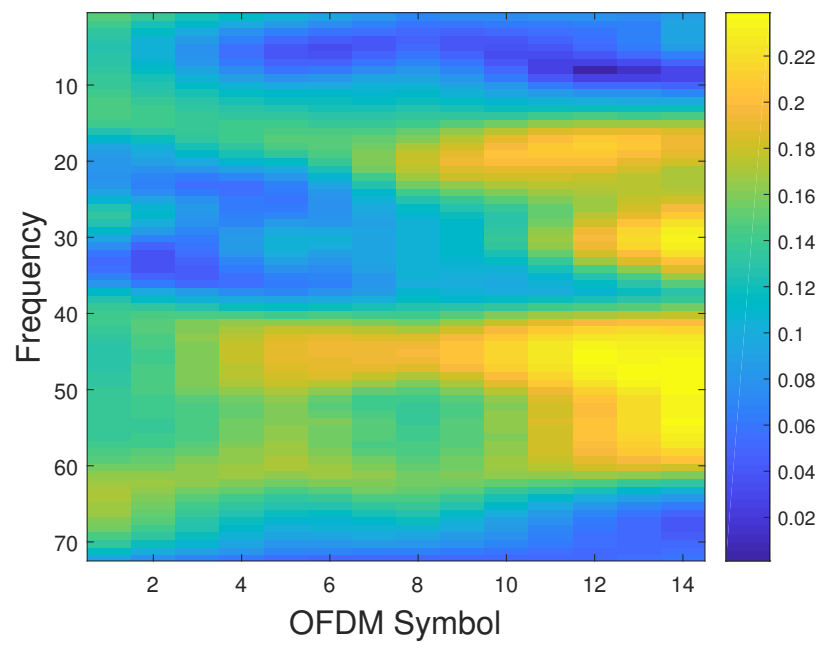

Figure 5.13: The magnitude of the estimate of the channel from transmitter antenna 1 . The CRS symbols are used and interpolated to get this estimate.

\subsubsection{Extract PBCH Resources}

Once we have the received grid and channel estimates for 2 TX antennas, it is time to extract the $\mathrm{PBCH}$ resources from these grids. The extracted $\mathrm{PBCH}$ symbols from our received grid is shown in Fig. 5.15 and the channel estimates from 2 transmitter antennas are shown in Fig. 5.16 and Fig. 5.17.

\subsubsection{PBCH Decoding}

The extracted $\mathrm{PBCH}$ resources from the received grid and channel estimates are used to obtain the 24 MIB bits in PBCH decoding block. MIB which is broadcasted through PBCH, carries three essential system information. They are [96]:

- System bandwidth

- System Frame Number (SFN)

- Physical Hybrid Automatic Repeat Request (HARQ) Indicator Channel (PHICH) Configuration 


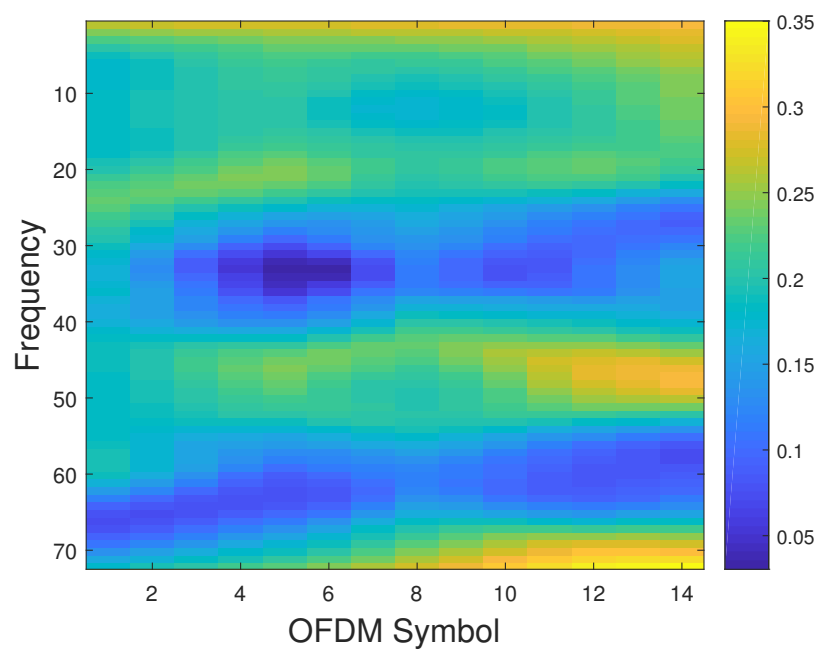

Figure 5.14: The magnitude of the estimate of the channel from transmitter antenna 2.

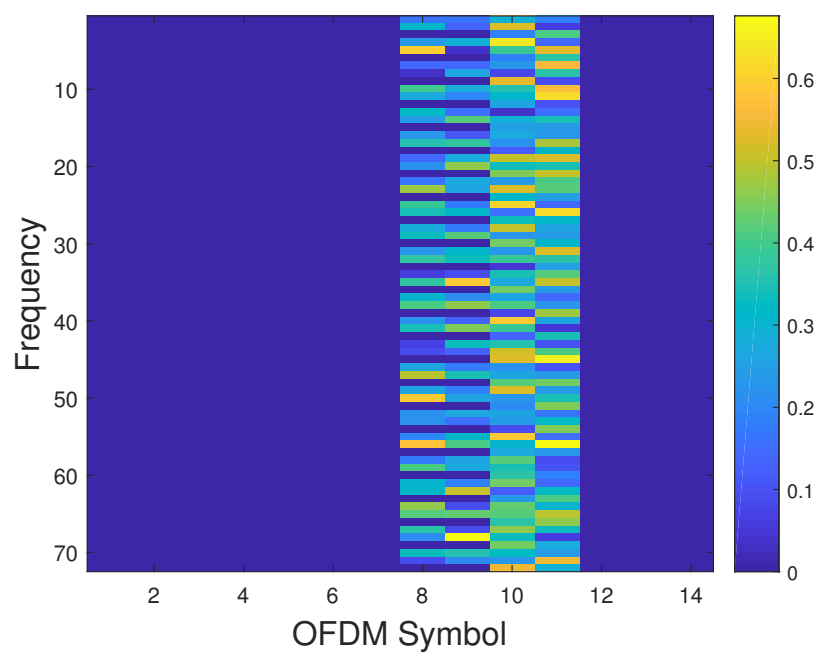

Figure 5.15: The locations and magnitudes of the PBCH symbols in subframe 0.

Successful decoding of the PBCH is extremely important to gain access to an LTE cell.

Once 24 MIB bits $\left(I_{M I B}\right)$ (14 information bits and 10 spare bits) are decoded successfully, system bandwidth is determined as follows:

$$
\operatorname{Ref}_{\mathrm{BW}}=4 \times \mathrm{I}_{\mathrm{MIB}}(1)+2 \times \mathrm{I}_{\mathrm{MIB}}(2)+\mathrm{I}_{\mathrm{MIB}}(3),
$$




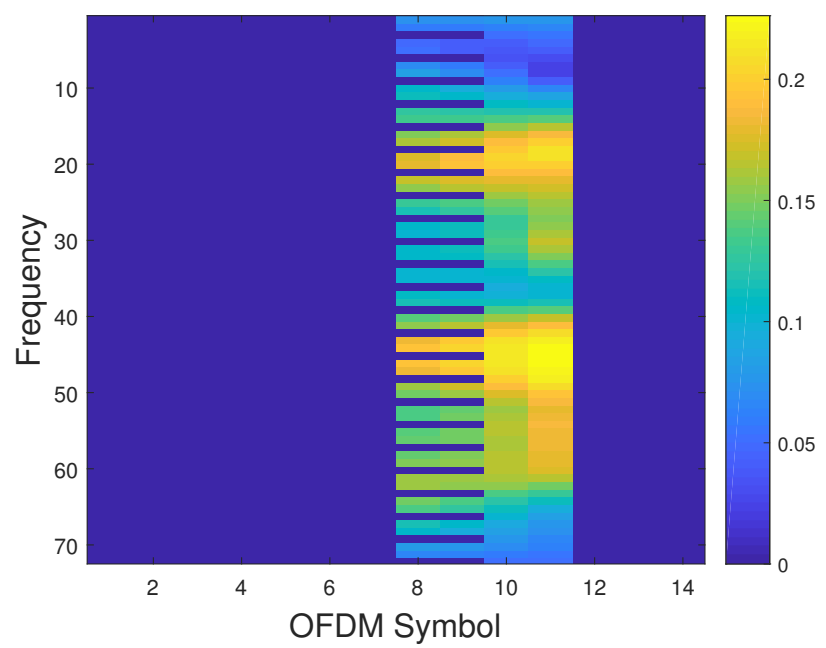

Figure 5.16: The magnitude of channel estimate from transmitter antenna 1 at $\mathrm{PBCH}$ symbols locations.

where, $I_{M I B}(i)$ is the $i^{\text {th }}$ bit in the MIB, $\operatorname{Ref}_{\mathrm{BW}} \in\{0,1,2,3,4,5\}$, and $\operatorname{Ref}_{\mathrm{BW}}=0$ corresponds to $1.4 \mathrm{MHz}$ or $6 \mathrm{RB}$ LTE transmission. Accordingly, other values in Ref $_{\mathrm{BW}}$ correspond to $3,5,10,15,20 \mathrm{MHz}$ of bandwidth respectively.

Based on the value of the $4^{\text {th }}$ MIB bit $I_{M I B}(4)$, PHICH duration is determined. If $I_{M I B}(4)=1$, PHICH duration is Extended; otherwise it is Normal. To identify PHICH resource type, $I_{\mathrm{MIB}}(5)$ and $I_{\mathrm{MIB}}(6)$ are used. Finally, SFN is detected by using 8 MIB bits, from $I_{\mathrm{MIB}}(7)$ to $I_{\mathrm{MIB}}(14)$.

Number of transmit antenna ports are determined using trial and error approach. This approach involves demodulation, unscrambling, de-rate matching, viterbi decoding and cyclic redundancy check $(\mathrm{CRC})$ processing. Detected bit sequence with proper CRC decoding will be identified as the $M I B$ information (24 bits). After that, number of resource blocks in the transmission, SFN and PHICH information is extracted. The PCI obtained earlier is verified in this stage by checking CRC after decoding MIB bits. 


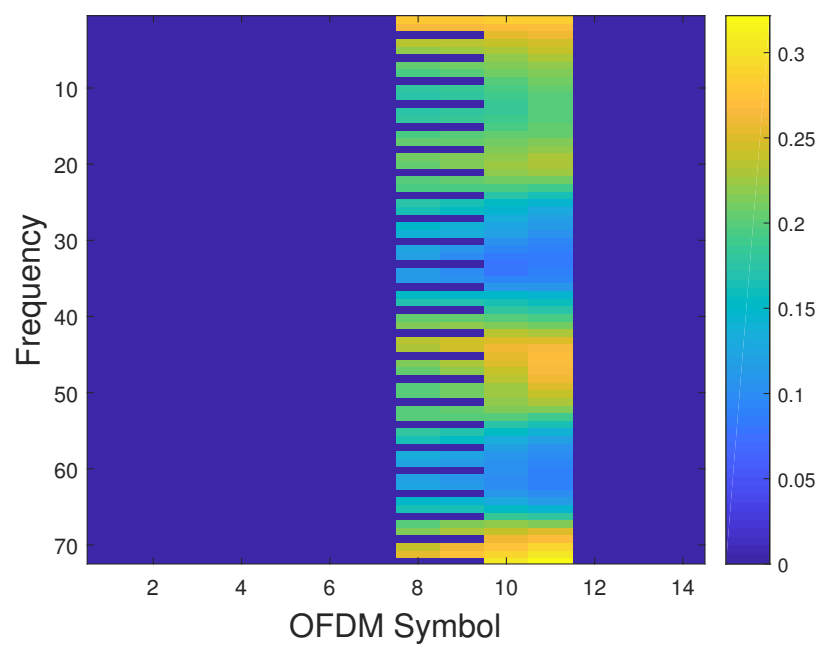

Figure 5.17: The magnitude of channel estimate from transmitter antenna 2 at $\mathrm{PBCH}$ symbols locations.

\subsubsection{Decoding SIB1 to uniquely identify the base station}

The maximum uniquely identifiable base station with PCI is limited to 504 and if it is not properly planned, can result in two neighboring cells having same PCI. This can cause a collision in the case of intra frequency cells and the UE cannot synchronize leading to service drop. Therefore it is important to have a unique identification which can be performed globally for the base station which is provided by E-UTRAN Cell Global Identifier (ECGI). It is composed of 3 bytes to identify Public Land Mobile Network (PLMN) Identity and 28 bits to identify the cell within that PLMN [97]. The base station broadcasts the ECGI information on the physical downlink shared channel (PDSCH) which is contained in the System Information Block type 1 (SIB1). Its scheduling is fixed similar to MIB but transmitted with a periodicity of $80 \mathrm{~ms}$ as shown in Fig. 5.18. The same versions of SIB1 is repeated for every $20 \mathrm{~ms}$ with different redundancy version (RV) which helps to ensure proper decoding of the turbo encoded PDSCH data in the HARQ process.

The overview of the decoding SIB1 information from the recorded measurements is shown in Fig, 5.19. The recorded signal is resampled according to the LTE sampling 


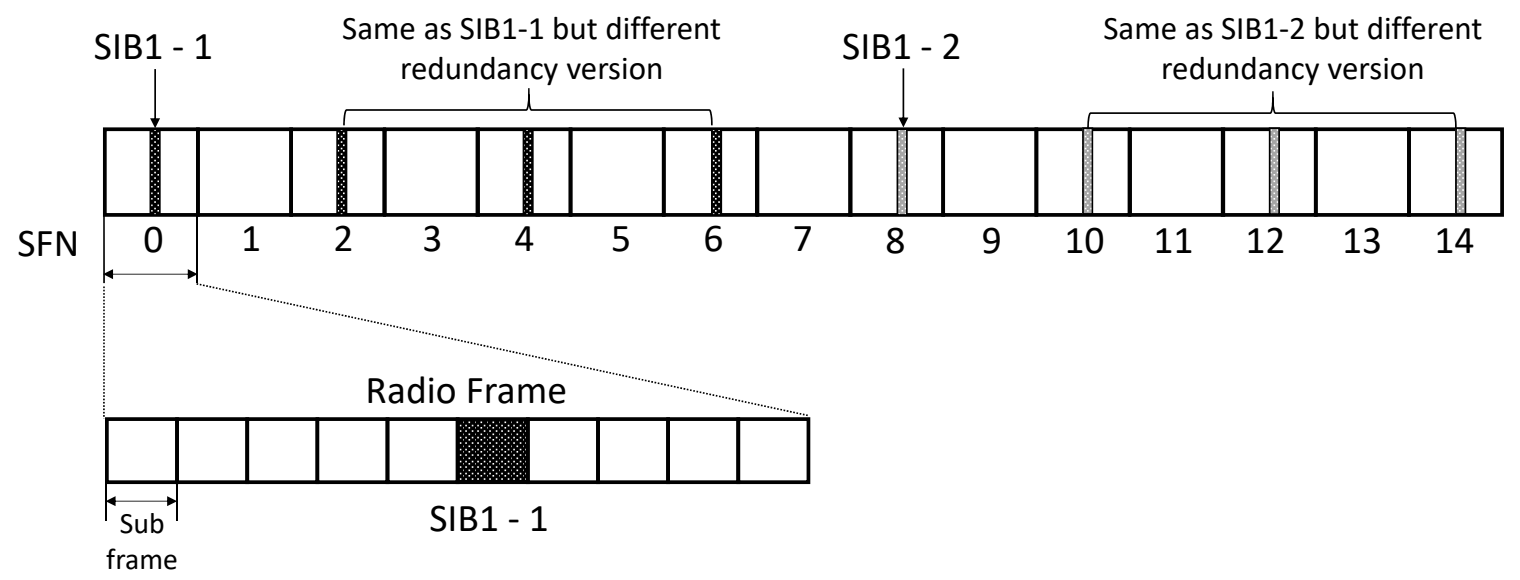

Figure 5.18: Scheduling of SIB1 information in time domain.

rate as shown in Table. 5.1. This is because the USRP N210 supports only integer decimation factor $N$ values and supported sampling rate is given by $\frac{100 e^{6}}{N}$. Therefore the sampling rate of the USRP is set more than the LTE sampling rate as show in Table. 5.1. Please note that the same sampling rate is used for both 15 and $20 \mathrm{MHz}$ signal, since the samples gets dropped due to overflow when it is greater than $25 \mathrm{MS} / \mathrm{s}$. Also, it is recommended to skip certain samples due to the settling time experienced after initiating the signal acquisition in the USRP. The $50 \mathrm{~ms}$ worth of samples are skipped and the rest are recorded in a CSV files which is explained in Section 5.2. In order to obtain SIB1 information, capture time duration $21 \mathrm{~ms}$ is enough in the good channel conditions, since it is repeated every $20 \mathrm{~ms}$ as shown in Fig. 5.18.

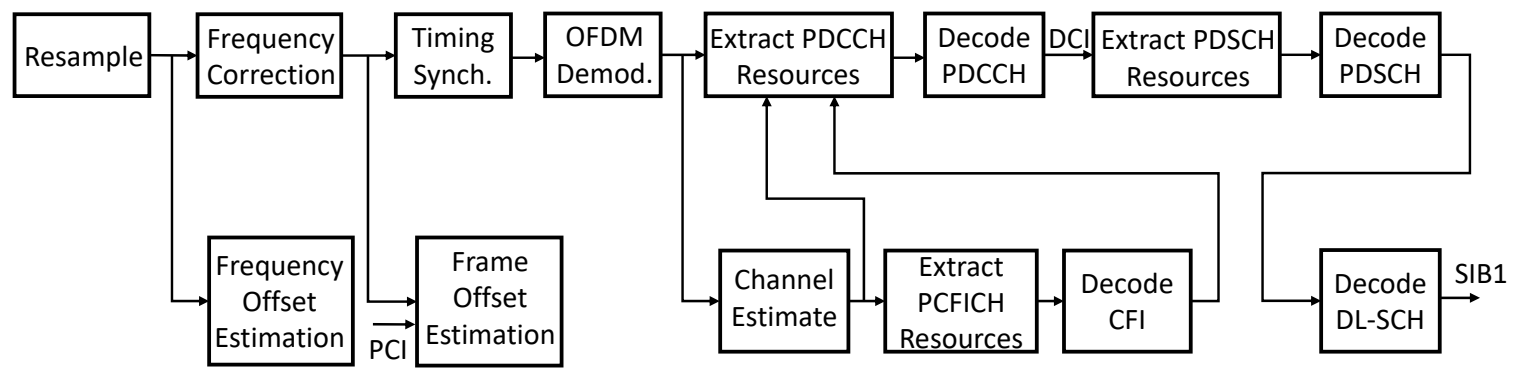

Figure 5.19: Decoding of SIB1 information. 
Table 5.1: Sampling Rates.

\begin{tabular}{|l|l|l|}
\hline $\begin{array}{l}\text { LTE Bandwidth } \\
\text { (MHz) }\end{array}$ & $\begin{array}{l}\text { LTE Sampling rate } \\
\text { (MS/s) }\end{array}$ & $\begin{array}{l}\text { USRP } \\
\text { Sampling rate }\end{array}$ \\
\hline 1.4 & 1.92 & $100 e^{6} / 50$ \\
\hline 5 & 7.68 & $100 e^{6} / 13$ \\
\hline 10 & 15.36 & $100 e^{6} / 6$ \\
\hline 15 & 23.04 & $100 e^{6} / 4$ \\
\hline 20 & 30.72 & $100 e^{6} / 4$ \\
\hline
\end{tabular}

After resampling, the frequency offset between the LTE base station and USRP is estimated and corrected, similar to the way we did for MIB decoding explained in Section 5.3.2. Further, for the timing synchronization, correlation is performed with the obtained PSS and SSS signals during cell search stage of the MIB decoding. The OFDM demodulation stage follows after the time and frequency synchronization and please note that the number of FFT points depends on the bandwidth of the LTE signal acquired after the MIB decoding stage.

The SIB1 information is transmitted on the transport channel called downlink shared channel (DL-SCH) and to perform decoding of the DL-SCH, we first determine how many OFDM symbols are transmitted in the physical downlink control channel (PDCCH) which is indicated by control format indicator (CFI). In LTE, values for the CFI can be 1,2, or 3 and therefore it is sufficiently robust to ensure it is decoded correctly to obtain PDCCH information. The CFI codewords are mapped to 16 resource elements (REs) in to four groups called resource element groups (REGs), where each group consists of 4 REs. These REs are distributed across the frequency domain excluding the resource element carrying reference symbols and their location have a specific offset related to PCI, in order to differentiate from the neighbouring cell. Once these locations are known, the PCFICH resources can be extracted. Next, the PCFICH resources are demodulated and then descrambling sequence which is again a function of PCI is applied to decode CFI. 
The PDCCH carries a message called downlink control information (DCI). It is transmitted in terms of control channel elements (CCE), where each CCE corresponds to nine REGs which are spread across the OFDM symbols and subcarriers and the number of occupied OFDM symbols is given by CFI. The REGs undergo interleaving and cyclic shifting before mapping on to resource elements. In addition CCEs are usually aggregated based on the PDCCH format, different PDCCH formats supported in LTE are shown in Table. 5.2.

Table 5.2: PDCCH formats.

\begin{tabular}{|l|l|l|l|}
\hline PDCCH format & Aggregated CCEs & \# of REGs & \# of PDCCH bits \\
\hline 0 & 1 & 9 & 72 \\
\hline 1 & 2 & 18 & 144 \\
\hline 2 & 4 & 36 & 288 \\
\hline 3 & 8 & 72 & 576 \\
\hline
\end{tabular}

In addition, the higher CCE aggregation can be used in the case of bad channel conditions, even if the PDCCH message having a certain format requires less number of CCEs. Since UEs is not explicitly mentioned about how many CCEs are transmitted, it has to blindly decode the message carried by the CCEs and this might cause additional processing burden and can consume lot of battery power in the case of larger bandwidths. Therefore in order to make decoding easier for the UE, the CCE location space is divided in to common search space and UE-Specific Search Space. The common search space carries control broadcast message common to all UEs, while the UE specific search space are specific to certain UEs. The common search space uses aggregation level which is limited to 4 or 8 shown in Table. 5.2.

The scheduling assignments for PDSCH carrying SIB1 information is given by compact and very compact signalling formats called DCI 1A and 1C respectively [97] and they are transmitted in common search space. Therefore UE sequentially extracts all the the PDCCH resources from the common search space and tries to decode for all 
possible DCI formats. In general, the cyclic redundancy check (CRC) attachment of the DCI is scrambled with radio network temporary identifier (RNTI) to differentiate modes of the UEs. Since DCI format $1 \mathrm{~A}$ or $1 \mathrm{C}$ is related to broadcast HARQ process, the SI-RNTI equal to 65535 is used which is common to all UEs. Now, the UE tries to descramble the CRC using the SI-RNTI and decodes the DCI. If it is successful then there will be no CRC error.

Further, PDSCH resources are extracted based on the resource assignments indicated by the DCI message. The decoding of PDSCH involves deprecoding, layer demapping and codeword separation, soft demodulation and descrambling [98]. The descrambling is performed using the SI-RNTI to obtain DL-SCH codewords. The steps involved in order to decode DL-SCH are rate recovery, turbo decoding, block concatenation and CRC calculations [98]. For the turbo decoding, the RV information shown in Fig. 5.18 is used for successful decoding to obtain SIB1 bits information. The following section explains the decoding of SIB1 bits to obtain ECGI information.

\subsubsection{Decoding the SIB1 information using ASN.1 compiler}

The ASN.1 compiler is used which basically converts the ASN.1 specifications to $\mathrm{C} / \mathrm{C}++$ executable code and its main job is to decode SIB1 bits stored .PER file which is the 3GGP RRC message standard. The SIB1 bits in .per file were the result of the drive test recording measurements performed around the NCSU campus. The compiler is installed on linux platform by following the steps given in [99] to obtain the executable code called progname. This decodes the bits in .per file to obtain BCCH-DL-SCH-Message shown in Fig. 5.20

The ECGI information consists of mobile country code (MCC), mobile network code (MNC), tracking area code (TAC) and cell identity (CID) as shown in Fig. 5.20. Using this information, we obtain base station location from the OpenCellid database 


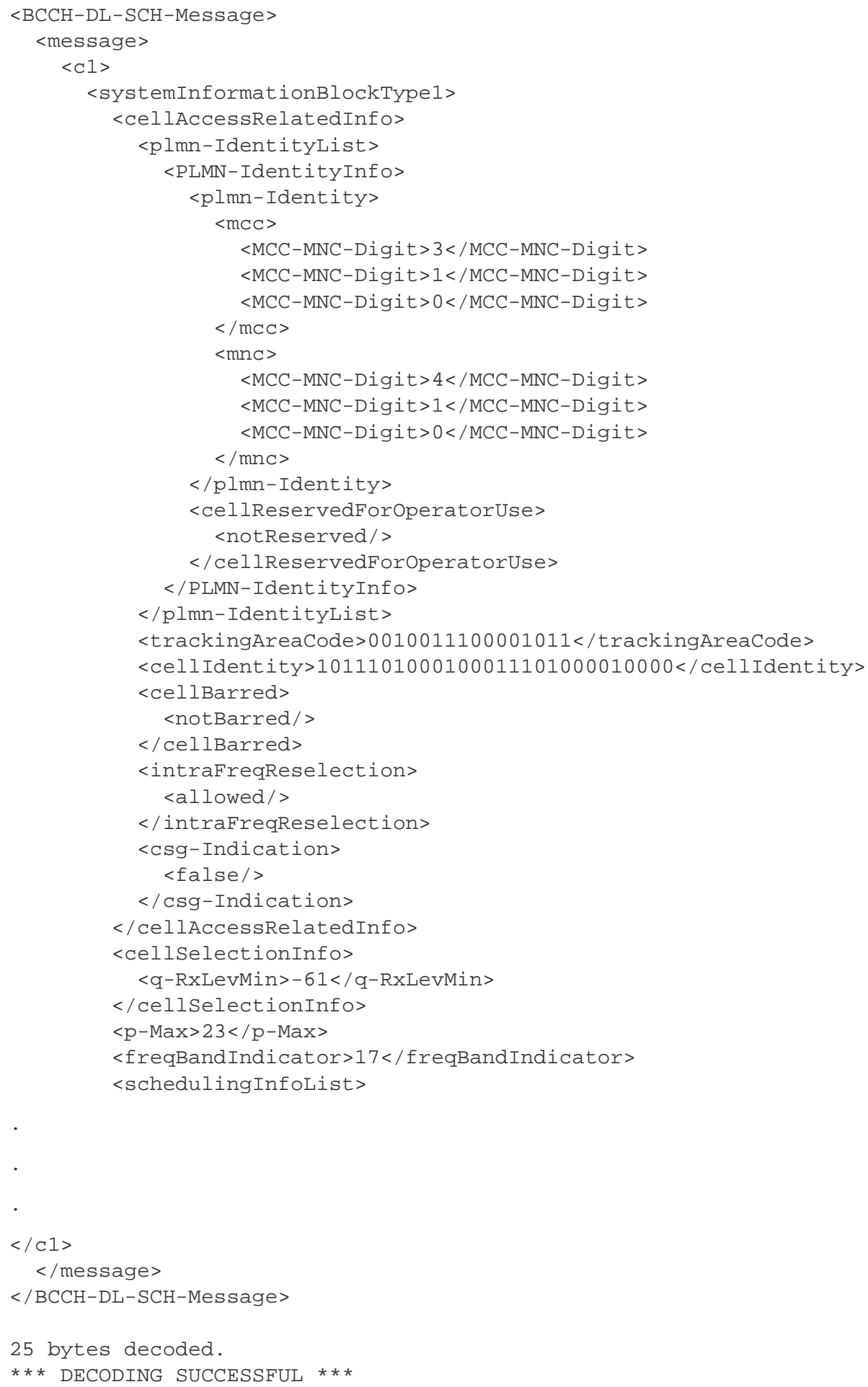

Figure 5.20: Decoded BCCH-DL-SCH message from SIB1 bits 
[100] and CellID finder [101]. Please note that the base station location obtained from these database are approximate.

\subsection{Experimental Results}

The drive test is performed around the NCSU campus and the USRP carrier frequency is hopped between frequencies $f=[739,2132] \mathrm{MHz}$ at each recorded GPS location. The locations are color mapped with RSRP values indicated by the colorbar as shown in Fig. 5.21a. The base station locations were obtained from the database based on the post processing results and plotted for the frequencies $f$ on the google map as shown in Fig. 5.21a.

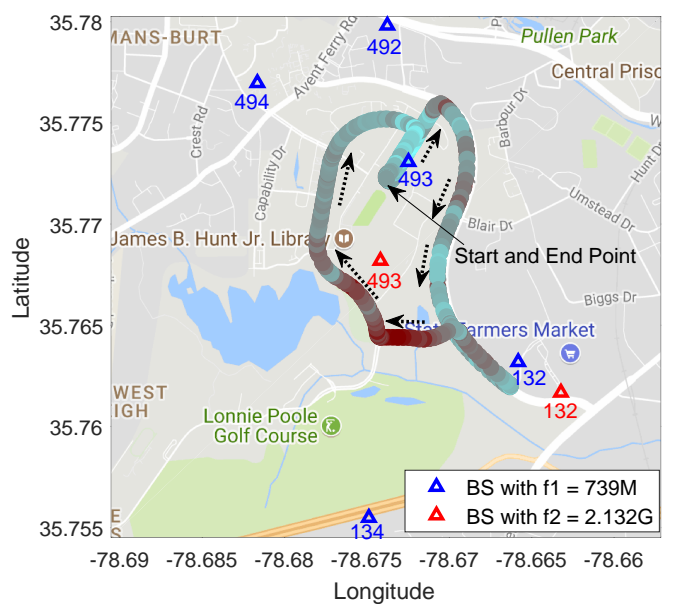

(a) Drive test scenario around NCSU campus.

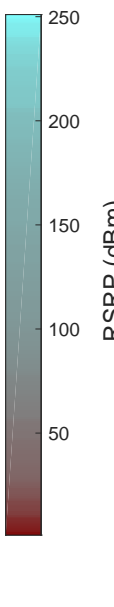

(b) RSRP variation with time for frequency $739 \mathrm{MHz}$

Figure 5.21: Drive test results around NCSU campus.

Initially, at the start of the drive test, the RSRP for the cell with PCI $=493$ is stronger and gradually decreases until the MIB decoding is no longer possible from the post processing, as a result there is discontinuity in the RSRP plot for the cell with PCI $=493$, similar explanation applies to RSRP plots for other cells which can be seen in Fig. 5.21b. Further, we can see RSRP peaks from other cells with PCI = 132/134 and again back to 493 and for the major part of the drive test it can be seen that 493 cell was the strongest cell. 


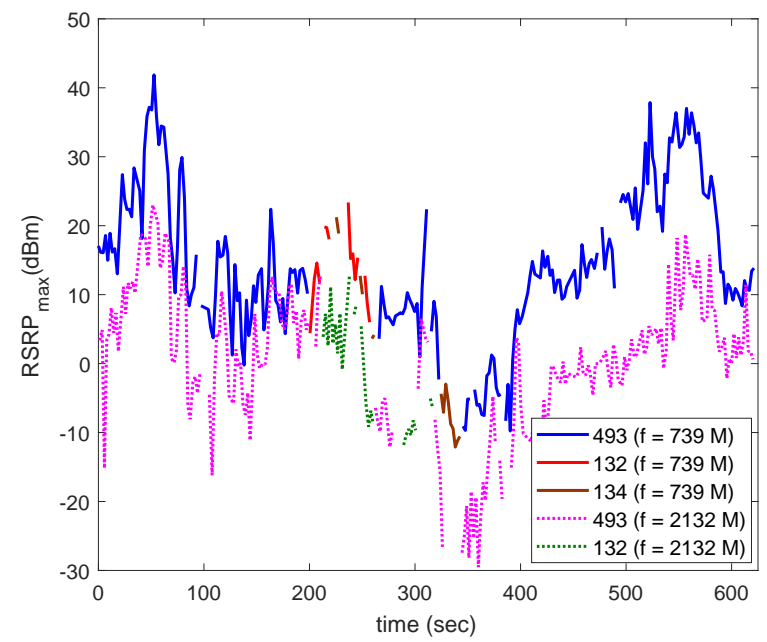

Figure 5.22: RSRP plots for the strongest base station.

The RSRP value of the strongest base station at all the recorded location is determined during the drive test shown in Fig. 5.21a. It can be observed that RSRP values are lower for the frequency $2.132 \mathrm{GHz}$ compared to $739 \mathrm{MHz}$ and this due to the increased pathloss for higher frequency or the base station is transmitting with lower power.

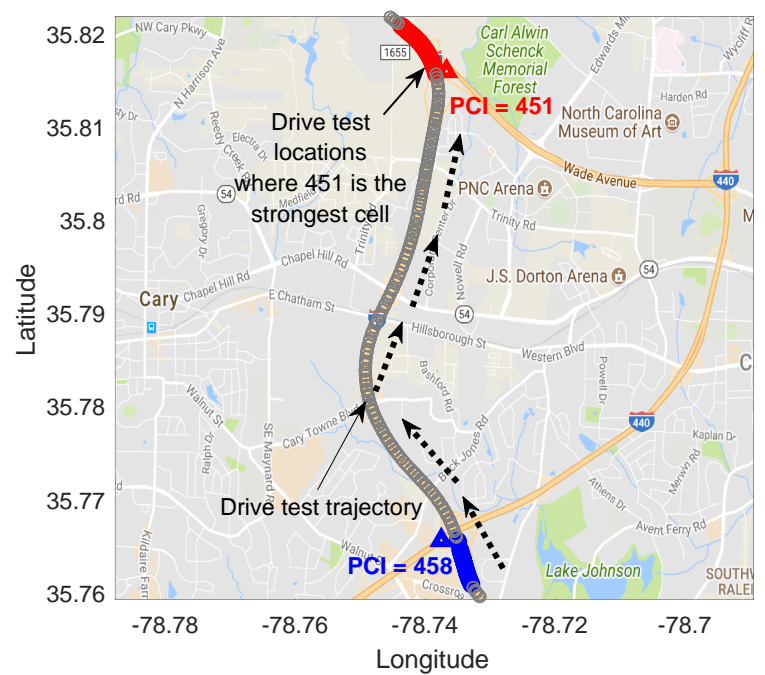

(a) Drive test scenario.

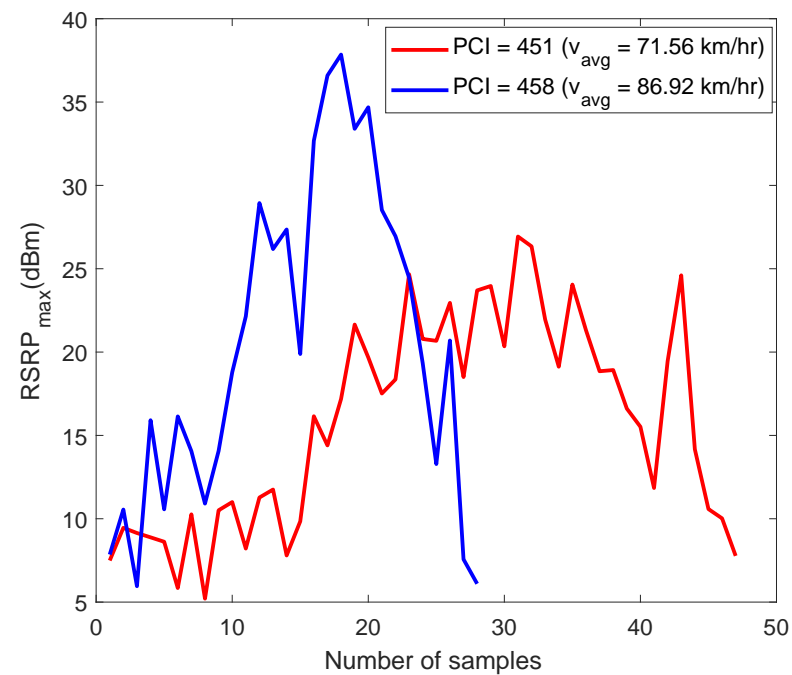

(b) RSRP variation as a function of velocity for frequency $739 \mathrm{MHz}$

Figure 5.23: Drive test results. 
Next we study the impact of velocity on the RSRP value of the strongest base station. The drive test scenario is shown in Fig. 5.23a. The blue and red points show the locations where the strongest cell is 458 and 451 respectively and the location of base stations is shown as triangle. When user approaches 458 and 451 cell with respective average velocity 86.92 and $71.56 \mathrm{~km} / \mathrm{hr}$ in the drive test, their RSRP profiles are shown in Fig. 5.23b. We observe that the RSRP peak width for 451 cell tends to be larger compared with 458 cell. This is because, the user tends to stay longer with 451 cell due to lower velocity $\left(v_{\text {avg }}=71.56 \mathrm{~km} / \mathrm{hr}\right)$ when compared with 458 cell.

Finally we investigate the distribution of base stations by performing drive test as shown in Fig. 5.24. The USRP carrier frequency is hopped between frequencies $f=[739,751,866.3,874.38,882.2,1967.5,1992.5,1982.5] \mathrm{MHz}$ at each recorded GPS location. We record time duration of $160 \mathrm{~ms}$ in order to acquire SIB1 information. The SIB1 decoding is performed and base station locations are obtained which are shown as black triangle in Fig. 5.24. We can observe that the base stations are densely deployed near the Raleigh downtown compared to countryside areas in the north.

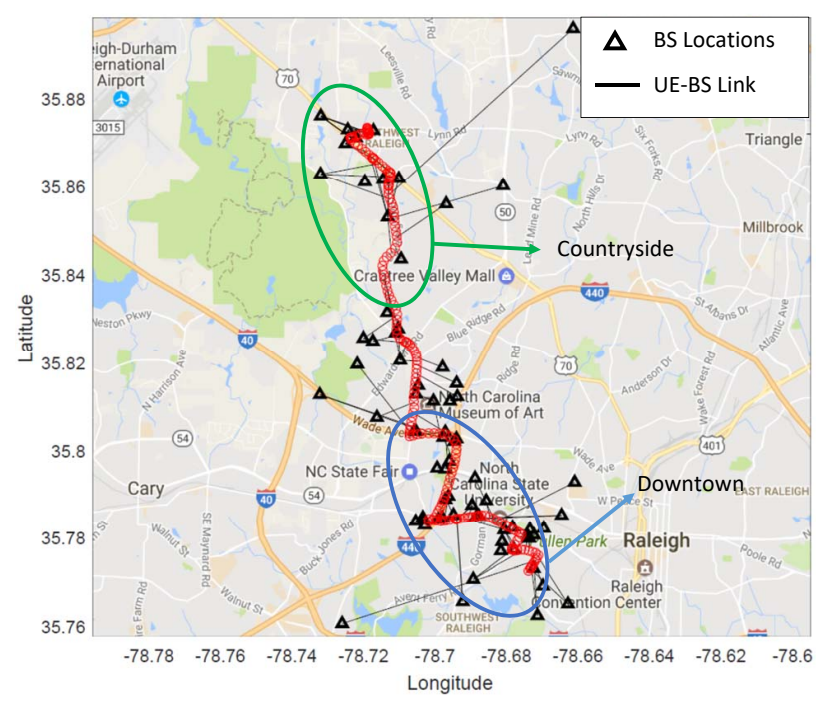

Figure 5.24: Distribution of base stations in the downtown and country side drive test scenarios. 


\section{CHAPTER 6 \\ CONCLUDING REMARKS}

The geometric abstraction is developed to derive analytic and semi-analytic expressions for HF and PP probabilities for scenarios with no-fading and fading in Chapter 3. Despite the several simplifying assumptions considered in the geometric model and framework, our findings still provide several useful insights corroborated by simulation results. In particular, the relationship between different parameters and HFs is explicitly captured, which is not possible in the case of earlier simulation studies available in the literature. The results show that fading may have significant impact on handover performance, and it increases the handover failure probability for all UE velocities. The handover performance for both MUEs and PUEs are improved when filtering sampling period is reduced. This improvement of handover performance is larger in the fading scenario. In particular, when tuning the filtering frequency, the HF performance is improved by 12.19 percent for MUEs in the fading scenario, compared with the 7.5 percent improvement in the no fading scenario.

We propose a fuzzy logic based game theoretical framework for energy efficiency improvement in heterogeneous networks in Chapter 4. Modified fuzzy decision rules were developed for the handovers and the target BS selection. Moreover, novel regret based game theoretical learning scheme was proposed for the energy efficient operation. It was shown that the proposed fuzzy-game theoretical technique improved the energy consumption significantly especially for the small number of active users considering the high user velocities with managing ping-pong handovers and cell loads.

The signal recordings are performed during drive test around NCSU campus. The recorded signal data is then post processed to obtain coverage of the base station indicated by RSRP. The impact of velocity on the RSRP is studied and it shows that

there are distinct RSRP peaks when user approaches the base station during drive 
test. The peak shapes vary as a function of velocity. It is observed that RSRP peak width decreases with increase in velocity. Further we observe the distribution of base stations and found that the it is densely deployed in urban areas compared to rural areas. 


\section{BIBLIOGRAPHY}

[1] Cisco, "Cisco VNI Mobile Report," Feb. 2017. [Online]. Available: http://www.cisco.com/c/en/us/solutions/collateral/service-provider/ visual-networking-index-vni/mobile-white-paper-c11-520862.html

[2] "Ericsson mobility report," Nov. 2016. [Online]. Available: https://www.ericsson. com/en/mobility-report

[3] 3GPP, "The 2020 vision for LTE," Feb. 2013. [Online]. Available: http: //www.3gpp.org/2020-vision-for-LTE

[4] - "3GPP LTE-A Standardisation in Release 12 and Beyond," Jan. 2013. [Online]. Available: http: //www.nomor.de/uploads/fd/24/fd24709a64bc490a083a8eba6d3cc2cb/ NoMoR_LTE-A_Rel12_and_Beyond_2013-01.pdf

[5] J. Andrews, S. Buzzi, W. Choi, S. Hanly, A. Lozano, A. Soong, and J. Zhang, "What will 5G be?" IEEE J. Select. Areas Commun. (JSAC), vol. 32, no. 6, pp. 1065-1082, Jun. 2014.

[6] Ascom, "HetNets: Opportunities and Challenges," Aug. 2013. [Online]. Available: http://www.nomor.de/uploads/fd/24/fd24709a64bc490a083a8eba6d3cc2cb/ NoMoR_LTE-A_Rel12_and_Beyond_2013-01.pdf

[7] D. Lopez-Perez, I. Guvenc, and X. Chu, "Mobility management challenges in 3GPP heterogeneous networks," IEEE Commun. Mag., vol. 50, no. 12, pp. 70-78, 2012.

[8] K. Dimou, M. Wang, Y. Yang, M. Kazmi, A. Larmo, J. Pettersson, W. Muller, and Y. Timner, "Handover within 3GPP LTE: design principles and performance," in Proc. IEEE Vehic. Technol. Conf. (VTC), Sep. 2009, pp. 1-5.

[9] T. Jansen, I. Balan, S. Stefanski, and T. Moerman, Ingrid "Weighted performance based handover parameter optimization in LTE," in Proc. IEEE Vehic. Technol. Conf. (VTC), no. 1, May 2011, pp. 1-5.

[10] H. Zhang, "A user mobility analysis assistive MRO algorithm for handover parameters optimization in LTE SON system," in Proc. IEEE Wireless Advanced (WiAd), Jun. 2012, pp. 143-148. 
[11] P. Munoz, R. Barco, and I. de la Bandera, "On the potential of handover parameter optimization for self-organizing networks," IEEE Trans. Vehic. Technol., vol. 62, no. 5, pp. 1895-1905, June 2013.

[12] D. Aziz, A. Ambrosy, L. T. Ho, L. Ewe, M. Gruber, and H. Bakker, "Autonomous neighbor relation detection and handover optimization in LTE," Bell Labs Technical Journal, vol. 15, no. 3, pp. 63-83, Dec. 2010.

[13] M. Anas, F. Calabrese, P.-E. Ostling, K. Pedersen, and P. Mogensen, "Performance analysis of handover measurements and layer 3 filtering for UTRAN LTE," in Proc. IEEE Int. Symp. Personal, Indoor and Mobile Radio Communications (PIMRC), 2007, pp. 1-5.

[14] D. Aziz and R. Sigle, "Improvement of LTE handover performance through interference coordination," in Proc. IEEE Vehic. Technol. Conf. (VTC), Barcelona, Spain, Apr. 2009, pp. $1-5$.

[15] W. Gao, B. Jiao, G. Yang, W. Hu, L. Chi, and J. Liu, "Mobility robustness improvement through transport parameter optimization in HetNets," in Proc. IEEE Int. Symp. Personal, Indoor and Mobile Radio Communications (PIMRC), Sept. 2013, pp. 101-105.

[16] S. Barbera, P. Michaelsen, M. Saily, and K. Pedersen, "Mobility performance of LTE co-channel deployment of macro and pico cells," in Proc. IEEE Wireless Commun. and Networking Conf. (WCNC), Apr. 2012, pp. 2863-2868.

[17] X. Gelabert, G. Zhou, and P. Legg, "Mobility performance and suitability of macro cell power-off in LTE dense small cell HetNets," in Proc. IEEE Int. Workshop on Computer Aided Modeling and Design of Communication Links and Networks (CAMAD), Sept. 2013, pp. 99-103.

[18] D. López-Pérez, I. Guvenc, and X. Chu, "Mobility enhancements for heterogeneous wireless networks through interference coordination," in Proc. IEEE Int. Workshop on Broadband Femtocell Technologies (co-located with IEEE WCNC), Paris, France, Apr. 2012.

[19] M. Simsek, M. Bennis, and İ. Güvenç, "Mobility management in HetNets: a learning-based perspective," EURASIP Journal on Wireless Communications and Networking, vol. 2015, no. 1, p. 26, 2015. [Online]. Available: http://dx.doi.org/10.1186/s13638-015-0244-2 
[20] — - "Learning based frequency- and time-domain inter-cell interference coordination in HetNets," IEEE Trans. Vehic. Technol., vol. 64, no. 10, pp. 4589-4602, Oct. 2015.

[21] S. Barbera, P. Michaelsen, M. Saily, and K. Pedersen, "Improved mobility performance in LTE co-channel HetNets through speed differentiated enhancements," in Proc. IEEE Globecom Workshops (GC Wkshps), Dec. 2012, pp. 426-430.

[22] S. Barbera, K. Pedersen, P. Michaelsen, and C. Rosa, "Mobility analysis for intersite carrier aggregation in LTE heterogeneous networks," in Proc. IEEE Vehic. Technol. Conf. (VTC), Sept. 2013, pp. 1-5.

[23] T.-S. Kim, R. Oh, S.-J. Lee, C.-H. Lim, S. Ryu, and C.-H. Cho, "Cell selection and trigger point decision for next generation heterogeneous wireless networking environment: Algorithm \& evaluation," in Information Science and Service Science (NISS), 2011 5th International Conference on New Trends in, vol. 2, Oct. 2011, pp. 247-254.

[24] A. Mehbodniya, F. Kaleem, K. Yen, and F. Adachi, "Wireless network access selection scheme for heterogeneous multimedia traffic," IET Networks, vol. 2, no. 4, pp. 214-223, Dec. 2013.

[25] Y. Peng, W. Yang, Y. Zhang, and Y. Zhu, "Mobility performance enhancements for LTE-Advanced heterogeneous networks," in Proc. IEEE Int. Symp. Personal, Indoor and Mobile Radio Communications (PIMRC), Sept. 2012, pp. 413-418.

[26] A. Merwaday and İ. Güvenç, "Handover count based velocity estimation and mobility state detection in dense HetNets," IEEE Trans. Wireless Commun., vol. 15, no. 7, pp. 4673-4688, July 2016.

[27] A. Merwaday, İ. Güvenç, W. Saad, A. Mehbodniya, and F. Adachi, "Sojourn time-based velocity estimation in small cell Poisson networks," IEEE Commun. Lett., vol. 20, no. 2, pp. 340-343, Feb. 2016.

[28] X. Lin, R. K. Ganti, P. Fleming, and J. G. Andrews, "Towards understanding the fundamentals of mobility in cellular networks," IEEE Trans. Wireless Commun., vol. 12, no. 4, pp. 1686 - 1698, Apr. 2013.

[29] S. Ramanath, V. Kavitha, and E. Altman, "Impact of mobility on call block, call drops, and optimal cell size in small cell networks," in Proc. IEEE Workshop on Indoor Outdoor Femto Cells (IOFC), Istanbul, Turkey, Sept. 2010, pp. 156-161. 
[30] F. Guidolin, I. Pappalardo, A. Zanella, and M. Zorzi, "A Markov-based framework for handover optimization in HetNets," in Proc. Ad Hoc Networking Workshop (MED-HOC-NET), June 2014, pp. 134-139.

[31] V. Nguyen, C. S. Chen, and L. Thomas, "Handover measurement in mobile cellular networks: analysis and applications to LTE," in Proc. IEEE Int. Conf. on Commun. (ICC), June 2011, pp. 1-6.

[32] D. López-Pérez, I. Guvenc, and X. Chu, "Theoretical analysis of handover failure and ping-pong rates for heterogeneous networks," in Proc. IEEE Int. Workshop on Small Cell Wireless Networks (co-located with IEEE ICC), Ottawa, Canada, Jun. 2012.

[33] K. Vasudeva, M. Simsek, D. Perez, and I. Guvenc, "Analysis of handover failures in heterogeneous networks with fading," IEEE Trans. Vehic. Technol, vol. PP, no. 99, pp. 1-15, 2016.

[34] K. Vasudeva, M. Simsek, and I. Guvenc, "Analysis of handover failures in HetNets with layer-3 filtering," in Proc. IEEE Wireless Commun. and Networking Conf. (WCNC), April 2014, pp. 2647-2652.

[35] K. Vasudeva, M. Simsek, D. Lpez-Prez, and I. Gven, "Impact of channel fading on mobility management in heterogeneous networks," in Proc. IEEE International Conference on Communication Workshop (ICCW), London, UK, Jun 2015, pp. 2206-2211.

[36] C. Mobile, "C-RAN: The Road Towards Green RAN," Oct. 2011. [Online]. Available: http://labs.chinamobile.com/cran/wp-content/uploads/CRAN_white_ paper_v2_5_EN.pdf

[37] TR 36.927, "Potential solutions for energy saving for E-UTRAN," 3GPP Technical Report, December 2015.

[38] E. Oh, K. Son, and B. Krishnamachari, "Dynamic base station switching-on/off strategies for green cellular networks," IEEE Trans. Wireless Commun., vol. 12, no. 5, pp. 2126-2136, May 2013.

[39] E. Oh, B. Krishnamachari, X. Liu, and Z. Niu, "Toward dynamic energy-efficient operation of cellular network infrastructure," IEEE Commun. Mag., vol. 49, no. 6, pp. 56-61, June 2011. 
[40] J. J. Q. Yu and V. O. K. Li, "Base station switching problem for green cellular networks with social spider algorithm," in Proc. IEEE Congress on Evolutionary Computation (CEC), July 2014, pp. 2338-2344.

[41] F. Alaca, A. B. Sediq, and H. Yanikomeroglu, "A genetic algorithm based cell switch-off scheme for energy saving in dense cell deployments," in Proc. IEEE Globecom Workshops (GC Wkshps), Dec 2012, pp. 63-68.

[42] A. Yildiz, T. Girici, and H. Yanikomeroglu, "A pricing based algorithm for cell switching off in green cellular networks," in Proc. IEEE Vehic. Technol. Conf. (VTC), June 2013, pp. 1-6.

[43] K. Son, H. Kim, Y. Yi, and B. Krishnamachari, "Base station operation and user association mechanisms for energy-delay tradeoffs in green cellular networks," IEEE J. Sel. Areas Commun., vol. 29, no. 8, pp. 1525-1536, September 2011.

[44] G. Cili, H. Yanikomeroglu, and F. R. Yu, "Cell switch off technique combined with coordinated multi-point (comp) transmission for energy efficiency in beyondlte cellular networks," in Proc. IEEE Int. Conf. Commun. (ICC), June 2012, pp. 5931-5935.

[45] A. Merwaday and I. Güvenç, "Optimisation of FeICIC for energy efficiency and spectrum efficiency in LTE-advanced HetNets," IET Electronics Letters, vol. 52, no. 11, pp. 982-984, 2016.

[46] A. Bousia, E. Kartsakli, A. Antonopoulos, L. Alonso, and C. Verikoukis, "Game theoretic approach for switching off base stations in multi-operator environments," in Proc. IEEE Int. Conf. Commun. (ICC), June 2013, pp. 4420-4424.

[47] A. Antonopoulos, E. Kartsakli, A. Bousia, L. Alonso, and C. Verikoukis, "Energy-efficient infrastructure sharing in multi-operator mobile networks," IEEE Commun. Mag., vol. 53, no. 5, pp. 242-249, May 2015.

[48] G. Micallef, P. Mogensen, and H. O. Scheck, "Cell size breathing and possibilities to introduce cell sleep mode," in Proc. European Wireless Conference (EW), April 2010, pp. 111-115.

[49] Z. Niu, Y. Wu, J. Gong, and Z. Yang, "Cell zooming for cost-efficient green cellular networks," IEEE Commun. Mag., vol. 48, no. 11, pp. 74-79, November 2010 . 
[50] R. Balasubramaniam, S. Nagaraj, M. Sarkar, C. Paolini, and P. Khaitan, "Cell zooming for power efficient base station operation," in International Wireless Communications and Mobile Computing Conference (IWCMC), July 2013, pp. $556-560$.

[51] S. Samarakoon, M. Bennis, W. Saad, and M. Latva-Aho, "Opportunistic sleep mode strategies in wireless small cell networks," in Proc. IEEE Int. Conf. Commun. (ICC), Jun. 2014, pp. 2707-2712.

[52] S. Zhou, J. Gong, Z. Yang, Z. Niu, and P. Yang, "Green mobile access network with dynamic base station energy saving," in Proc. ACM MobiCom, vol. 9, no. 262, 2009, pp. 10-12.

[53] J. Wu, Y. Zhang, M. Zukerman, and E. K. N. Yung, "Energy-efficient basestations sleep-mode techniques in green cellular networks: A survey," IEEE Commun. Surveys Tuts., vol. 17, no. 2, pp. 803-826, Secondquarter 2015.

[54] M. Ismail, W. Zhuang, E. Serpedin, and K. Qaraqe, "A survey on green mobile networking: From the perspectives of network operators and mobile users," IEEE Commun. Surveys Tuts., vol. 17, no. 3, pp. 1535-1556, thirdquarter 2015.

[55] A. H. Arani, M. J. Omidi, A. Mehbodniya, and F. Adachi, "A handoff algorithm based on estimated load for dense green $5 \mathrm{~g}$ networks," in Proc. IEEE Global Communications Conference (GLOBECOM), Dec 2015, pp. 1-7.

[56] R. Razavi, S. Klein, and H. Claussen, "A fuzzy reinforcement learning approach for self-optimization of coverage in lte networks," Bell Labs Technical Journal, vol. 15, no. 3, pp. 153-175, 2010. [Online]. Available: http://dx.doi.org/10.1002/bltj.20463

[57] — - "Self-optimization of capacity and coverage in lte networks using a fuzzy reinforcement learning approach," in Proc. IEEE International Symposium on Personal, Indoor and Mobile Radio Communications, Sept 2010, pp. 1865-1870.

[58] K.-L. Tsai, H.-Y. Liu, and Y.-W. Liu, "Using fuzzy logic to reduce ping-pong handover effects in lte networks," Soft Computing, vol. 20, no. 5, pp. 1683-1694, 2016. [Online]. Available: http://dx.doi.org/10.1007/s00500-015-1655-z

[59] J. S. R. Jang, "ANFIS: adaptive-network-based fuzzy inference system," IEEE Transactions on Systems, Man, and Cybernetics, vol. 23, no. 3, pp. 665-685, May 1993. 
[60] K. C. Foong, C. T. Chee, and L. S. Wei, "Adaptive network fuzzy inference system (anfis) handoff algorithm," in Proc. International Conference on Future Computer and Communication(ICFCC), April 2009, pp. 195-198.

[61] P. Muoz, R. Barco, and I. de la Bandera, "On the potential of handover parameter optimization for self-organizing networks," IEEE Trans. Vehic. Technol., vol. 62, no. 5, pp. 1895-1905, Jun 2013.

[62] P. Munoz, R. Barco, I. de la Bandera, M. Toril, and S. Luna-Ramirez, "Optimization of a fuzzy logic controller for handover-based load balancing," in Proc. IEEE Vehic. Technol. Conf. (VTC), May 2011, pp. 1-5.

[63] A. Merwaday and I. Güvenç, "Handover count based velocity estimation and mobility state detection in dense HetNets," IEEE Trans. Wireless Commun., vol. 15, no. 7, pp. 4673-4688, 2016.

[64] A. Merwaday, I. Güvenç, W. Saad, A. Mehbodniya, and F. Adachi, "Sojourn time-based velocity estimation in small cell poisson networks," IEEE Commun. Lett., vol. 20, no. 2, pp. 340-343, 2016.

[65] K. Vasudeva, S. Dikmese, . Gven, A. Mehbodniya, W. Saad, and F. Adachi, "Fuzzy logic game-theoretic approach for energy efficient operation in hetnets," in IEEE International Conference on Communications Workshops (ICC Workshops), May 2017, pp. 552-557.

[66] K. Vasudeva, S. Dikmese, . Gven, A. Mehbodniya, W. Saad, and F. Adachi, "Fuzzy-based game theoretic mobility management for energy efficient operation in hetnets," IEEE Access, vol. 5, pp. 7542-7552, 2017.

[67] C.-T. Chen, C.-T. Lin, and S.-F. Huang, "A fuzzy approach for supplier evaluation and selection in supply chain management," International Journal of Production Economics, vol. 102, no. 2, pp. 289 - 301, 2006. [Online]. Available: http://www.sciencedirect.com/science/article/pii/S0925527305000782

[68] A. Mehbodniya, F. Kaleem, K. Yen, and F. Adachi, "Wireless network access selection scheme for heterogeneous multimedia traffic," Networks, IET, vol. 2, no. 4, pp. 214-223, Dec. 2013.

[69] M. P. Wylie-Green and T. Svensson, "Throughput, capacity, handover and latency performance in a 3gpp lte fdd field trial," in IEEE Global Telecommunications Conference GLOBECOM, Dec 2010, pp. 1-6. 
[70] M. Palola, M. Matinmikko, J. Prokkola, M. Mustonen, M. Heikkil, T. Kippola, S. Yrjl, V. Hartikainen, L. Tudose, A. Kivinen, J. Paavola, and K. Heiska, "Live field trial of licensed shared access (lsa) concept using lte network in $2.3 \mathrm{ghz}$ band," in IEEE International Symposium on Dynamic Spectrum Access Networks (DYSPAN), April 2014, pp. 38-47.

[71] J. H. Schaffner, A. Bekaryan, H. J. Song, T. Talty, D. Carper, E. Yasan, and A. Duzdar, "A drive test measurement approach to characterize on-vehicle 2x2 ltemimo antennas," in IEEE-APS Topical Conference on Antennas and Propagation in Wireless Communications (APWC), Aug 2014, pp. 85-88.

[72] L. C. Gimenez, M. C. Cascino, M. Stefan, K. I. Pedersen, and A. F. Cattoni, "Mobility performance in slow- and high-speed lte real scenarios," in IEEE 83rd Vehicular Technology Conference (VTC Spring), May 2016, pp. 1-5.

[73] R. Irmer, H. p. Mayer, A. Weber, V. Braun, M. Schmidt, M. Ohm, N. Ahr, A. Zoch, C. Jandura, P. Marsch, and G. Fettweis, "Multisite field trial for lte and advanced concepts," IEEE Communications Magazine, vol. 47, no. 2, pp. 92-98, February 2009.

[74] X. Yin, M. Tian, L. Ouyang, X. Cheng, X. Cai, L. Tian, J. Chen, and P. Yang, "Modeling city-canyon pedestrian radio channels based on passive sounding in in-service networks," IEEE Transactions on Vehicular Technology, vol. 65, no. 10, pp. 7931-7943, Oct 2016.

[75] S. Kumar, E. Hamed, D. Katabi, and L. Erran Li, "Lte radio analytics made easy and accessible," in ACM SIGCOMM Computer Communication Review, vol. 44, no. 4. ACM, 2014, pp. 211-222.

[76] "Evrytania LTE cell scanner/tracker," 2012. [Online]. Available: https: //github.com/Evrytania/LTE-Cell-Scanner

[77] "Lte Cell Scanner," 2013. [Online]. Available: https://github.com/JiaoXianjun/ LTE-Cell-Scanner

[78] N. Rupasinghe and I. Guven, "Capturing, recording, and analyzing lte signals using usrps and labview," in SoutheastCon 2015, April 2015, pp. 1-7.

[79] 3GPP TSG RAN WG2 70b, R2-104017, "Mobility support to pico cells in the co-channel HetNet deployment," Samsung, June 2010. [Online]. Available: http: //www.3gpp.org/ftp/tsg_ran/WG2_RL2/TSGR2_70bis/Docs/R2-104017.zip 
[80] TS 36.300, "E-UTRA Overall description," 3GPP Technical Report, June 2015.

[81] TS 36.214, "E-UTRA Physical layer Measurements," 3GPP Technical Report, June 2015.

[82] TS 36.331, "E-UTRA RRC Protocol Specification," 3GPP Technical Report, June 2015.

[83] TR 36.839, "Mobility Enhancements in Heterogeneous Networks," 3GPP Technical Report, June 2011.

[84] TS 25.302, "Services provided by the physical layer," 3GPP Technical Report, September 2014.

[85] "Bertrand Paradox." [Online]. Available: http://web.mit.edu/urban_or_book/ www/book/chapter3/3.3.2.html

[86] T. 25.996, "Spatial channel model for multiple input multiple output (mimo) simulations (release 12)," 3GPP Technical Report, Sep. 2014.

[87] G. Auer, V. Giannini, C. Desset, I. Godor, P. Skillermark, M. Olsson, M. A. Imran, D. Sabella, M. J. Gonzalez, O. Blume, and A. Fehske, "How much energy is needed to run a wireless network?" IEEE Wireless Commun., vol. 18, no. 5, pp. 40-49, Oct. 2011.

[88] R. Aumann, "Subjectivity and correlation in randomized strategies," Journal of Mathematical Economics, vol. 1, no. 1, pp. 67-96, 1974. [Online]. Available: http://EconPapers.repec.org/RePEc:eee:mateco:v:1:y:1974:i:1:p:67-96

[89] S. Hart and A. Mas-Colell, "A simple adaptive procedure leading to correlated equilibrium," Econometrica, vol. 68, no. 5, pp. 1127-1150, 2000. [Online]. Available: http://EconPapers.repec.org/RePEc:ecm:emetrp:v:68: y:2000:i:5:p:1127-1150

[90] K. Tanaka and M. Sugeno, "Stability analysis and design of fuzzy control systems," Elsevier Fuzzy Sets and Systems, vol. 45, no. 2, pp. 135-156, 1992.

[91] H. O. Wang, K. Tanaka, and M. Griffin, "Parallel distributed compensation of nonlinear systems by Takagi-Sugeno fuzzy model," in Proc. Int. Joint Conf. Fuzzy Systems and Int. Fuzzy Engineering Symp., vol. 2, 1995, pp. 531-538. 
[92] E. R. Website, Mar. 2014. [Online]. Available: https://www.ettus.com/product/ details/UN210-KIT

[93] Sparkfun, "Nmea reference manual," 2017. [Online]. Available: https: //www.sparkfun.com/datasheets/GPS/NMEAReferenceManual1.pdf

[94] Niviuk, "Lte frequency band," 2017. [Online]. Available: http://niviuk.free.fr/ lte_band.php

[95] TS 36.101, "Evolved Universal Terrestrial Radio Access (E-UTRA); User Equipment (UE) radio transmission and reception," June 2017.

[96] MatLab, "Cell Search, MIB and SIB1 Recovery." [Online]. Available: http:// www.mathworks.com/help/lte/examples/cell-search-mib-and-sib1-recovery.html

[97] S. Sesia, I. Toufik, and M. Baker, LTE : From Theory to Practice, 2nd Edition. John Wiley and Sons Ltd, 2011.

[98] MatLab, "LTE DL-SCH and PDSCH Processing Chain." [Online]. Available: http://www.mathworks.com/help/lte/examples/ lte-dl-sch-and-pdsch-processing-chain.html

[99] Peng, "ASN.1 compiler to generate the LTE-RRC message decoding program." [Online]. Available: http://blog.csdn.net/peng_yw/article/details/22437251

[100] OpenCellid, "Open database of cell towers," 2017. [Online]. Available: https://opencellid.org/

[101] CellID Finder, "Basestation location coordinates with mcc, mnc, lac and cid." [Online]. Available: http://cellidfinder.com/ 
VITA

\section{KARTHIK VASUDEVA}

December 6, 1986

2008

$2008-2010$

2012-2014

2014-2017

2015

2015

2016-
Born, Mysuru, India

B.E., Telecommunication Engineering

B.M.S College of Engineering

Bangalore, India

Associate IT Consultant

ITC Infotech India Ltd

Kolkata, India

Teaching Assistant

Florida International University

Miami, Florida

Research Assistant

Florida International University

Miami, Florida

Doctoral Candidate

Florida International University

Miami, Florida

Visiting Research Assistant

Tohoku University

Sendai, Japan

Visiting Research Assistant

North Carolina State University

Raleigh, North Carolina

PUBLICATIONS AND PRESENTATIONS 
[1] K. Vasudeva, M. Simsek, D. Perez, and I. Guvenc, "Analysis of handover failures in heterogeneous networks with fading," IEEE Trans. Vehic. Technol, vol. PP, no. 99, pp. 1-15, 2016.

[2] K. Vasudeva, S. Dikmese, . Gven, A. Mehbodniya, W. Saad, and F. Adachi, "Fuzzy-based game theoretic mobility management for energy efficient operation in hetnets," IEEE Access, vol. 5, pp. 7542-7552, 2017.

[3] K. Vasudeva, M. Simsek, and I. Guvenc, "Analysis of handover failures in HetNets with layer-3 filtering," in Proc. IEEE Wireless Commun. and Networking Conf. (WCNC), Istanbul, Turkey, Apr. 2014, pp. 2647-2652.

[4] K. Vasudeva, M. Simsek, D. Lpez-Prez, and I. Gven, "Impact of channel fading on mobility management in heterogeneous networks," in Proc. IEEE International Conference on Communication Workshop (ICCW), London, UK, Jun. 2015, pp. 2206-2211.

[5] K. Vasudeva, B. S. Ciftler, A. Altamar, and I. Guvenc, "An experimental study on RSS-based wireless localization with software defined radio," in Proc. IEEE Wireless and Microwave Technology Conference (WAMICON), Tampa, FL, Jun. 2014, pp. 1-6.

[6] K. Guevara, M. Rodriguez, N. Gallo, G. Velasco, K. Vasudeva, and I. Guvenc, "UAV-based GSM network for public safety communications," in Proc. IEEE SoutheastCon, Ft. Lauderdale, FL, Apr. 2015, pp. 1-2.

[7] K. Vasudeva, S. Dikmese, . Gven, A. Mehbodniya, W. Saad, and F. Adachi, "Fuzzy logic game-theoretic approach for energy efficient operation in hetnets," in IEEE International Conference on Communications Workshops (ICC Workshops), May 2017, pp. 552-557. 